\title{
An Emperor Penguin Optimization and Particle Swarm Optimization Control algorithm for PV with ZSI in Grid Connected System
}

Vidhya M ( $\sim$ vidya182020@gmail.com )

Anna University Chennai

Senthil Kumar R

Bannari Amman Institute of Technology

\section{Research Article}

Keywords: grid connected PV system, hybrid approach, ZSI, SEPIC converter, EPO and PSO

Posted Date: April 21st, 2021

DOl: https://doi.org/10.21203/rs.3.rs-440660/v1

License: (c) (1) This work is licensed under a Creative Commons Attribution 4.0 International License.

Read Full License 


\title{
An Emperor Penguin Optimization and Particle Swarm Optimization Control algorithm for PV with ZSI in Grid connected System
}

\author{
${ }^{1 *}$ Mrs. Vidhya. M, ${ }^{2}$ Dr. Senthil Kumar. R \\ $1 *$ Research scholar, Department of EEE, Anna University, Chennai, TamilNadu, India. \\ vidya182020@gmail.com \\ ${ }^{2}$ Professor, Department of EEE, Bannari Amman Institute of Technology, Sathyamanglam. \\ TamilNadu, India.
}

\begin{abstract}
In the paper, a distributed controller for the analysis of SEPIC and Z-Source Inverter (ZSI) based grid-connected Photovoltaic (PV) system. The PV systems are modeled, designed and implemented to given maximum power output with the help of proposed controller. In the paper, a distributed controller is designed to provide maximum output power and to working by changing irradiance and providing various range of active power. An enhanced controller is specified as the Fractional Order PID (FOPID) controller with aid of hybrid approach. The hybrid approach is consisting of Emperor Penguin Optimization (EPO) and Particle Swarm Optimization (PSO). FOPID controller is the advanced PID controller and it gives a better output and robustness than the traditional PID controller. To enhance the performance of the FOPID, the gain parameters are optimized with the utilization of hybrid approach. For achieving the optimal power management and maximum power tracking, the objective function is defined and specified their constraints also. Here, two various methods have been deliberated to changing the inverter operation with constant irradiance and varied irradiance. The proposed method is tested by using MATLAB/Simulink and it is contrasted with the previously developed methods like base model, Ant Colony Optimization
\end{abstract} (ACO) and PSO algorithm.

Keywords: grid connected PV system, hybrid approach, ZSI, SEPIC converter, EPO and PSO

\section{Introduction}


Now a day, integration of sustainable energy resources with distribution system for satisfaction of increasing demands in grid side [1]. The renewable sources are also increasing more attention because of reducing emission and pollutions. Different kinds of renewable sources are available in market like Photovoltaic (PV), Wind Turbine (WT), Hydroelectric, Geothermal, in addition Biomass and so on [2,3]. From the different types of sustainable energy sources, PV is considered as maximum important sustainable energy source because it has more advantages such as easy to be harvested, clean and noise free. Recent years, increasing interest is installing PV in the house rooftop for satisfying energy demand of the system because of different incentive policies [4]. The PV can be placed in each home to generating power from sunlight energy which avoided extra placement of PV panel and easy to implement. The generating power used to many purposes especially home appliances and extra demand factor [5]. The installment cost of PV is high but maintenance cost at low which generates high power based on load demand. The PV relates to grid system; grid side demand also meets by consumption of generated power [6].

The PV generation system may be affected by environmental conditions which mostly weather behaviour. The high amount of sunlight energy is generated high amount of power similarly low amount of sunlight energy produces low amount of power [7,8]. Based on irradiance condition, the PV is generated power which used to different applications mostly grid side demand factors. The irradiance level provides generated power of PV; irradiance is directly proportional to generated power. Due to environmental conditions, the generated power of PV is totally affected. On that time, grid side demand cannot be satisfied [9]. To solve those problems, energy storage device is introduced in PV with grid connected system. The energy storage device plays main role of grid connected system if required demand is high. The energy storage device with PV can be combined for meeting load demand in distributed system [10,11]. At critical conditions of PV can be solved with the consumption of energy storage system. The different AC-DC converters can be utilized to enable best performance of grid connected PV system. In the PV system, LUO converter based maximum power is obtained. The PV with battery system must be improved with proper power management, State of Charge (SOC) controller in addition Maximum Power Point Technique (MPPT) [12].

Many different controllers are developed by researchers such as supervisory controller, coordinated controller and management controller. All methods are having different shortcoming 
which must be improved by different developed method. Recently, optimization techniques are utilized to enhance power management, charging with discharging controller and MPPT controller [13]. The different meta-heuristic algorithms are already used to power management and MPPT controller such as Firefly Algorithm (FA) [14], Grey Wolf Optimization (GWO) and Particle Swarm Optimization (PSO), and so on. Each optimization algorithm is working based on hunting behaviour of their family. The power management controller can be computed load difference among generation side and distribution system load demand. The load demand variations are compensated with the consumption of battery [15]. In the battery SOC controller is essential to enhance their lifetime which also managed by controller. In the PV, MPPT controller is essential to manage environmental conditions. The controller is managed by FOPID controller which tuned by optimization algorithms.

\section{Literature Review}

Many different methods have been developed to achieve power management and MPPT controller in grid connected PV system. Few related works are reviewed in this section,

Md Alamgir Hossain et al. [16] has developed modified PSO algorithm for the energy management towards compute optimal control of battery of microgrid structure. And PSO algorithm can be modified which consists of changing cost function towards best design of discharging and charging processes. The best control was completed through expressing a cost function that was completely suitable examined and formerly a changeable penalty function to achieve best cost function was developed. The different case studies with various conditions were led to find the efficiency of developed cost function. The developed cost function can be able to minimize the operational cost through $12 \%$ contracted with the general cost function based on the 96 hours load demand. The implementation results disclose the appropriateness of smearing the legalized PSO algorithm for compensate power management.

Kallol Roy et al. [17] have been developed Recurrent Neural Network (RNNN) and the mixture of Ant Lion Optimizer (ALO) for finding energy scheduling in the microgrid structure. The optimal power management programming of electrical system by minimization of generation cost and best consumption of sustainable energy resources of storage systems, PV and WT energy generation systems. The main objective was the developed method utilized to the enhancement of 
optimal progress of the micro sources aimed at reducing the electricity generation cost by hourly day optimum operation related to the real time preparation. And developed technique was talented to investigate the economic time in need of constraints in addition technical operating constraints. The load demand response was compensated with the utilization of the developed method by reducing the minimum energy cost. The load demand response can be computed based on the RNN and additional performance indices are analyzed for customer response.

Abedini et al. [18] have developed an ensured combination Particle Swarm Optimization with Gaussian Mutation (GPSO-GM) estimation based ideal administration of MG system with including supportable force sources. Their ideal administration philosophy of PV/wind/diesel free mixture systems for giving required energy in self-sufficient MG. An enhancement issue was anticipated restricting the capital endeavor and fuel costs of the system. To deal with the proposed improvement issue a figuring, named GPSO-GM was made. Two administrators, specifically change and ensured union, were included to PSO to support finding more precise results and accelerating assessments.

Tidjani et al. [19] have proposed a fuzzy logic supervisor based active damping procedure and smart power management the board for enhancement of standalone MG framework. MG was pulling in extensive consideration as an answer for energy inadequacy, particularly, in far off territories. A MG was characterized as a gathering of interconnected burdens and different appropriated generators that were typically incorporated by means of Voltage Source Convertors (VSC) and had the option to work in both grids connected and island-mode. To improve power quality and force energy the board dependability, MG needs streamlining as far as control and size decrease of parts. To make a few commitments, their paper breaks down three perspectives, for example, building up a methodology of dynamic damping procedure dependent on numerical model of VSC with no extra sensors, decreasing the size of the yield LC channel by moving the recurrence and changing the dynamic damping coefficient and building a solid fluffy rationale director for brilliant force the executives.

L. Zacharia1 et al. [20] have developed two optimization algorithms implemented for managing the microgrid operation in islanded mode and grid connected mode. The management algorithm can be attained based on the priorities and objectives in each mode. The stable operation and optimal operation at each mode the presented method can regulate the charging and 
discharging level, defined the generation level of the energy storage system and shed loads. In the microgrid operation, the optimal power management can be attained based on the two-optimization algorithm. The stable and optimal operation of microgrid was of crucial importance and can be a challenging task. The effectiveness of the proposed optimal and stable operation of microgrid, proposed scheduling was implemented by real time simulation where different transitions among the islanded mode and grid connected were analyzed. The experimental results were indicating which the dev eloped method was able to optimize efficiently the energy flows of the microgrid even under different transitions.

\section{Proposed System Model}

In this paper, a distributed control strategy is proposed to deal with voltage fluctuation problems through proposed PV inverter control in order to improve the power management of distribution networks with high PV integration. The power management is essential task for improving the performance of the grid combined PV system. The proposed grid connected PV consists of PV panel, DC-DC converter, Inverter and battery. The proposed architecture of the power management in grid connected PV system is illustrated in the figure 1. The grid connected PV structure can be able to compensate the DC and AC loads. In the architecture, the PV system is utilized to generate the power for compensate the load demand of the grid side demand factor [21]. The PV is mainly working based on the sunlight energy can be converted in to the electrical energy. The renewable resources of PV are act as the primary generation of power for attaining the load demand of the system. The battery is act as a secondary generation of power for reimburse the load demand in the grid connected PV system. Additionally, battery can be used to store the excess power from the generation side and provide the power to meet the load demand. The battery should be maintained the charging and discharging control because it participates to compensate the load demand of the grid connected PV system. 


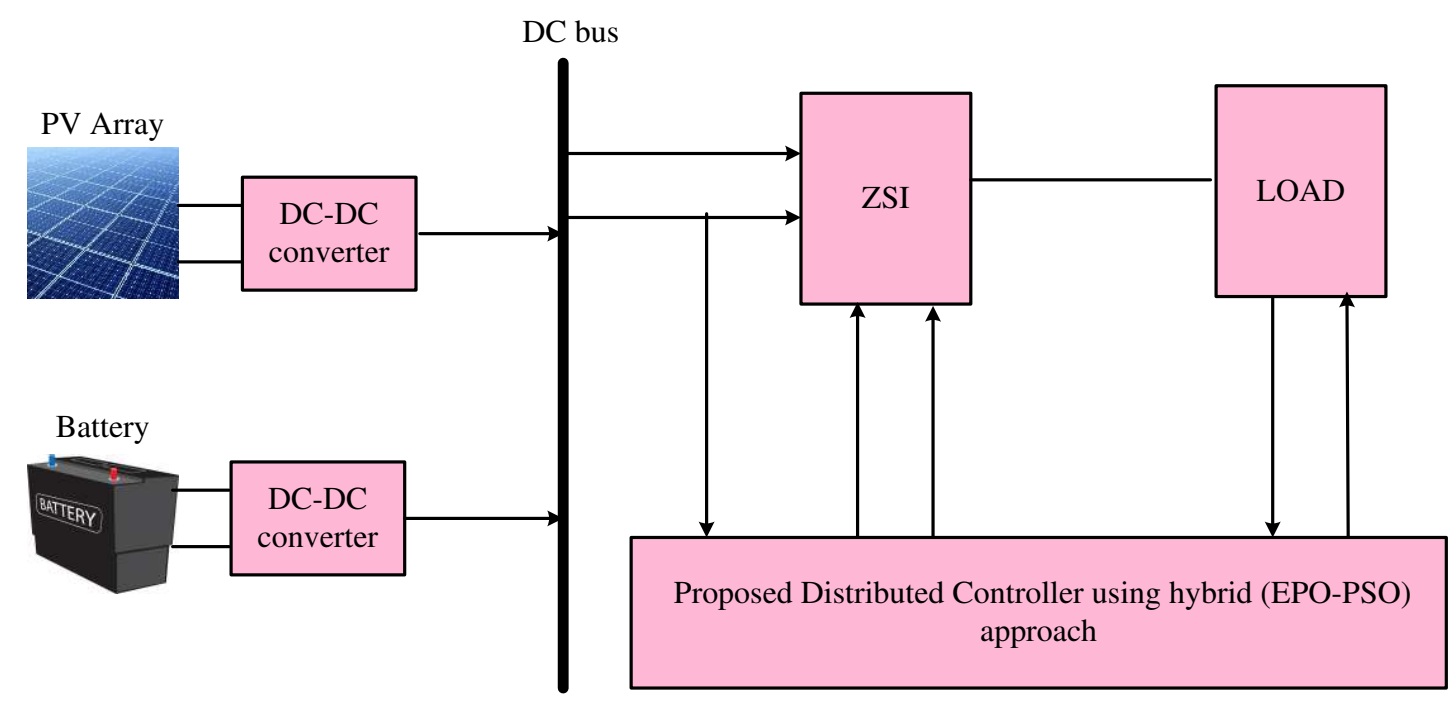

Figure. 1 proposed method system model

In our architecture, the PV is selected for generating the power and battery is used for storage power to attain the load demand. The DC-DC converters are utilized to boost the renewable energy source of PV, the PV can be affected by the environmental conditions. In our proposed methodology, SEPIC converter is selected to excerpt greatest power from grid connected PV system. And MPPT controller is exploited towards enhance the extreme power from the PV system during various environmental conditions. Additionally, different irradiance condition of PV system should affect the performance of system which must be reduced by MPPT controller. After extraction of maximum power from PV system, the designed ZSI can be utilized to forward generated power to AC load in grid side. The power management should be maintained in the PV with grid connected structure for satisfy the linear and nonlinear load. The proposed distributed controller is used to accomplish the power among the generation with load demand of the system. The proposed distributed controller gain parameters should be optimized for enhancing the performance of the grid connected PV system. The gain constraints are selected with the help of the hybrid algorithm which contains EPO and PSO algorithm. The modelling and proposed controller with optimization algorithm is detail presented in the following section.

\subsection{Modelling of PV}

In the part, an overall numerical depiction of PV cell is introduced. PV cells are known to be electrical machines that transform light energy into electrical energy. The energy obtained from 
PV boards that are sun oriented is realistic. This semiconductor board ingests daylight photons and delivers electrons from molecules, providing a potential contrast. This induces a stream of flow in the material to miss the possible contrast and the power is caught [22] afterwards. Figure 2 applies to an identical PV framework circuit model model. It speaks of an equivalent circuit of the overall model consisting of a photocurrent, a diode, and a parallel resistor communicating a leakage current, and a series resistor reflecting an internal resistance from the current flow.

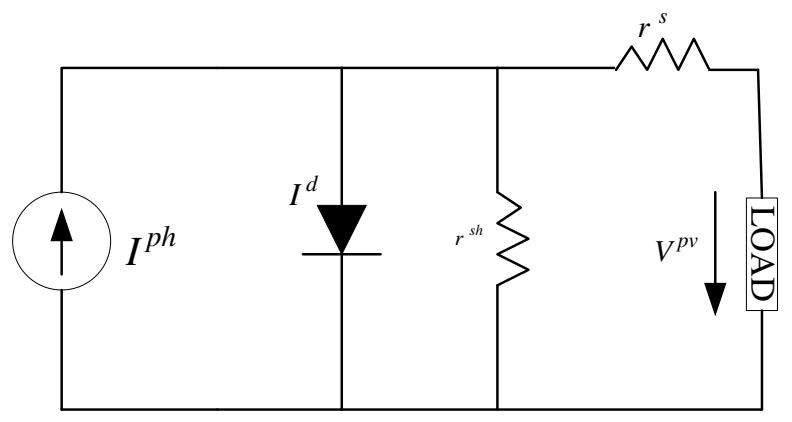

Figure. 2 I-V characteristics of the PV system with equivalent circuit model

The voltage and current of a solar cell is denoted as the following,

$$
I_{c}^{P V}=\frac{I_{p h}}{1-C^{-d}}\left[\exp ^{\frac{V_{o c}}{a}}\left(\frac{q v+q r_{v} I}{N K T}\right)-1\right]-\frac{V+R_{s} I}{R_{s h}}
$$

Where,

$V_{o c}$ is the open circuit voltage

$R_{s h}$ is a shunt resistance

$\mathrm{N}$ is an ideal factor

$\mathrm{T}$ is the cell working temperature

$q=\left(1.6 * 10^{-19} C\right)$ is denoted as electron charge

$I$ noted as the current

As indicated by the illumination ranges, sun-based board models are expressed and controlled. Each sunlight-based board can be controlled at a solitary moment at different illumination levels 
between $600 \mathrm{~W} / \mathrm{m} 2$ and $1000 \mathrm{~W} / \mathrm{m} 2$. In view of the illumination, the yield power of the sun-based board has been modified and it can be maintained very well by using the correct age of control signals for the DC-DC converter. The MPPT regulator modifies the DC-DC converter's obligation cycle (D) to maintain the working point of the PV boards, generally at the MPP. Here, as per their data sources, the proposed MPPT-based regulator is used to adjust the duty pattern of the DC-DC converter. In the grid race associated PV framework, the BESS is utilized to repay load demand when PV neglects to fulfill load need. The displaying of the BESS is clarified in the underneath segment.

\subsection{Modelling of BESS}

The BESS was planned to balance the demand for loads during times of unavailability of renewable energy sources. In this paper, in addition to the demand based on the following formula, battery capacity is planned in accordance with the desired autonomy date [23]. The desired autonomy day parameter means the number of days a battery bank would be able to satisfy the energy demand of PV systems that are presented mathematically as follows,

$$
B^{\text {cap }}=\frac{\text { autonomy demand. } p^{l}}{\eta^{\text {inverter }} \cdot \eta^{B} \cdot D O D}
$$

The SOC ( $\mathrm{t}$ ) can be defined as a quantity of charge and the battery can be defined as the maximum and minimum SOC. At maximum charge should be utilized to evaluate the load demand of the system. The PV energy system are considered as the renewable energy sources, which are highly specific in nature in addition it generates a surplus or deficit energy. The surplus or deficit energy describes the power delivered and absorbed through the battery energy storage system given by below,

$$
P^{B}(t)=\left(P^{P V}(t)-\frac{P^{l}(t)}{\eta^{i n v}}\right)
$$

Where, $P^{P V}(t)$ can be represented as total power produced by the $\mathrm{PV}$ and $P^{l}(t)$ can be represented as the energy demand system and $\eta^{i n v}$ can be represented as the inverter efficiency.

The two conditions should be checked $P^{B}(t)<0$ which indicates the power generation and $P^{B}(t)>0$ is an indication which power generation has been surpassed energy demand. The 
battery charging occurs when $S O C(t)<S O C$ or when the $P^{P V}(t)>P^{l}(t)$ in addition therefore the SOC $(\mathrm{t})$ of the battery given below equation,

$$
\operatorname{SOC}(t)=\operatorname{SOC}(t-1) \cdot(1-\sigma)+\left[\left(P^{P V}(t)-\frac{P^{l}(t)}{\eta^{\text {inv }}}\right)\right] \times \eta^{B}
$$

Similarly, the decrease of the power generation, the Its Battery Storage is above its minimum allowable range and battery then moves to discharge the energy stored in it to compensate energy demand related to the generated power.

$$
\operatorname{SOC}(t)=\operatorname{SOC}(t-1) \cdot(1-\sigma)+\left[\left(\frac{P^{l}(t)}{\eta^{i n v}}-\left(P^{P V}(t)\right)\right] \times \eta^{B}\right.
$$

Where, $\eta^{B}$ can be expressed as the efficiency and self-discharge rate and $\sigma$ can be described as the efficiency respectively. The DC-DC converter can be utilized to enable the maximum power from PV system. After extraction of PV maximum power which send to grid distribution system through the consumption of ZSI. The modelling and mode of operation of ZSI is presented in below parts.

\subsection{Modeling of SEPIC converter}

SEPIC converter where it is normally same as classical buck boost converter. It has non-inverting output (output voltage has the same similar polarity as the input voltage). SEPIC converter where it normally has two modes of operations. In proposed system we use continuous mode of operation.

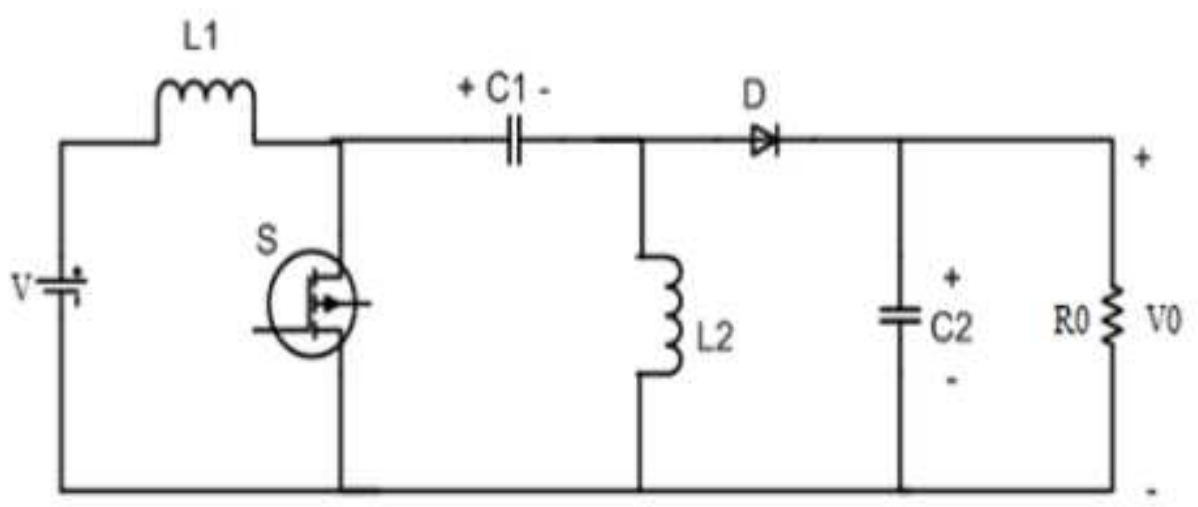

Figure. 3 Design of SEPIC converter 
In Figure 3shows the circuit diagram of a SEPIC converter when the current through inductor $L 1$ is continuous, it will be in a mode of continuous conduction. SEPIC converter exchanges energy between the inductor and capacitor where it converts from one voltage form to another voltage [24]. With the help of switch $S 1$ where it helps to control exchanged energy. The switch is generally used is MOSFET where it has high impedance and low voltage than the bipolar junction transistor. When switch S1is closed, inductor $L 1$ will store the energyas the current through it increases. Voltage drop across $L 1$ isequal to the input voltage. Diode is opened.L2 Charged bycapacitor $C 1$. $C 2$ discharges through the load and gives the output. The switch $S 1$ is open and diode is closed. Inductor $L 1$ where current decreases and charging capacitor $C 1$. The current through inductor $L 2$ decreases and charges capacitor $C 2$.Capacitor $C 2$ provides the output at load. In the SEPIC converter, when the switch $\mathrm{S}$ is $\mathrm{ON}$, the inductor $L 1$ gets charged from the input sourceVin and inductor $L 2$ gets charged from the discharging capacitor $C 1$. This makes the diode $D$ to be in reverse biased condition and the capacitor $C 2$ will supply power to the load. When the switch $\mathrm{S}$ made OFF, the inductor $L 2$ discharges through the diode which makes it to conduct and the capacitors are charged from the inductors.

\subsection{Modeling of Z-source Inverter}

The ZSI is utilized to control the power management of the grid associated PV framework, the ZSI is comprises of an X shape LC network which can be utilized as boot or buck the info dc voltage. The ZSI buck or boost operation is relies upon the time of shoot through zero state under the switching cycle [25]. The ordinary voltage source inverter comprises of eight admissible switching state that are six dynamic switches and two distinctive zero states. Yet, the ZSI may like as zero states for all various switches are gated on shoot through state also the state can be utilized to achieve boosting the dc input voltage. In any case, the ZSI likewise have the six distinct states with

two zero states furthermore it has the shoot through express that is prohibited in customary inverters can used to boost the DC input voltage. The working method of the ZSI is delineated in the beneath figure4. 


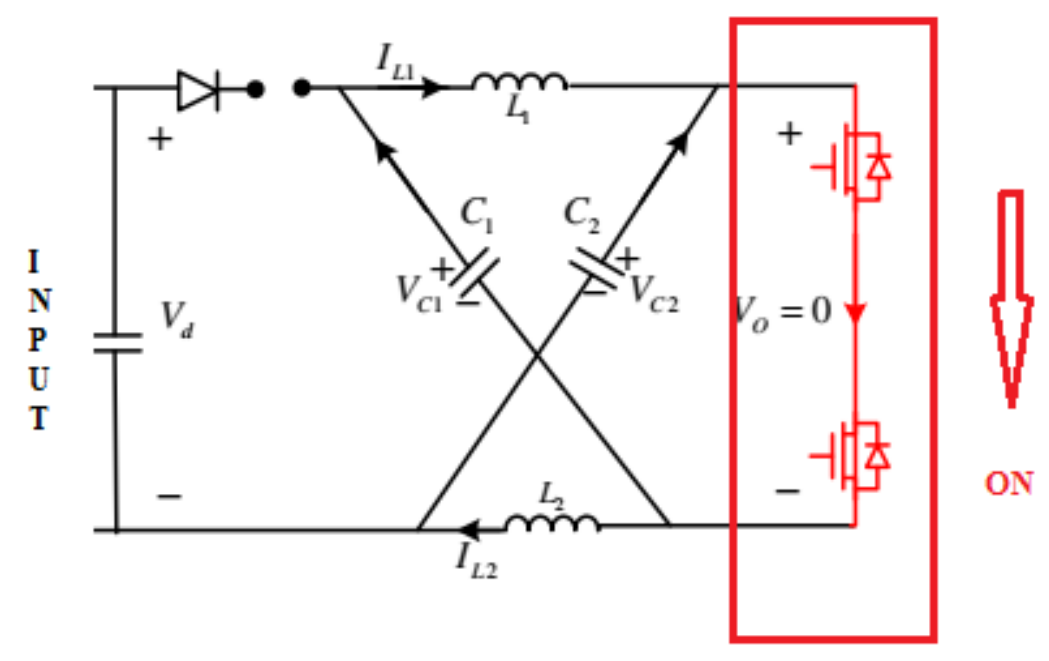

(a)

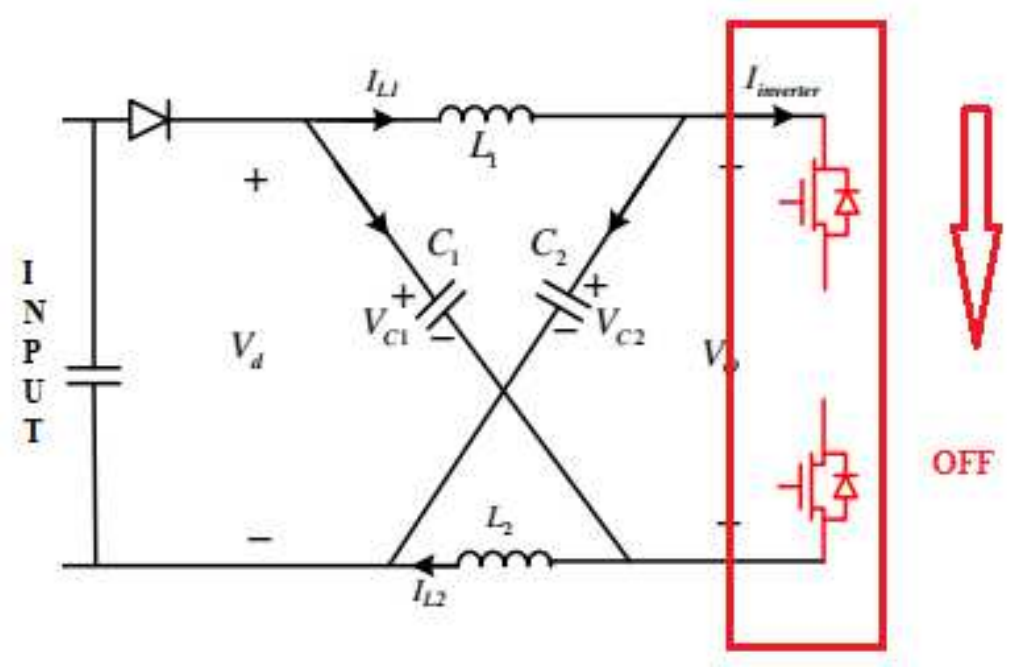

(b)

Figure.4 ZSI equivalent circuit diagram of (a) State of Shoot through and non-shoot through

The equivalent circuit of the ZSI have the two unique methods of activity, for example, shoot through state and non-shoot through state individually. In the shoot through express, the six switches of the ZSI are turned ON for a time spans of $T$ in each exchanging interval $(t)$, the overall relations for the ZSI are accomplished as,

$$
V^{C 1}=V^{C 2}=V^{C}=\frac{1-\frac{T}{t}}{1-2 \frac{T}{t}} V^{i n p u t}
$$




$$
\left\{\begin{array}{cc}
V^{\text {OUT }}=\frac{1}{1-2 \frac{T}{t}} V^{\text {input }}=S V^{\text {input }} & \text { State of Non }- \text { shoot } \\
V^{\text {OUT }}=0 & \text { state of }- \text { shoot }
\end{array}\right.
$$

Where, $V^{\text {input }}$ can be spoken to as info voltage of impedance organization, $V^{\text {OUT }}$ can be spoken to as yield voltage of impedance organization, $S$ can be spoken to as the boosting element of the ZSI, $V^{\text {OUT }}$ can be spoken to as the capacitors voltages of impedance network that are the comparable due to circuit evenness.

The ZSI descriptors and examination depend on suppositions which inductance of inductor in the impedance network is extraordinary fulfillment to stabilize the inductor current practically consistent. In the conditions, inductance is exceptionally little, and inductor current wave can be ceases or wave that presumptions can't be fulfilled. To conquer the high ripple, shoot through and non-shoot through states are introduced in the ZSI. In the ZSI, the third mode introduced when the inductor current scopes to line current of ZSI half. The fourth and fifth method of activity, the inverter is changed to a no dynamic exchanging state. In this paper, the ZSI is utilized to disseminated control of the matrix associated PV framework. The disseminated control of lattice associated PV framework is a best control technique which legitimately acknowledges total changing conditions of inverter to quick track the reference boundaries [26]. This method doesn't utilize the traditional zero states exchanging states. Notwithstanding, the fourth and fifth activity methods of ZSI is utilized don't occur while the third mode will introduce if the inductor current lessens to half of the stage current. In the method of activity, the input diode in the ZSI directing cycle can be halted likewise, the inductor current stands equivalent to capacitors voltage esteems. At last, introduced of this working mode gives a more modest shoot through period time. At the same time, a little estimation of shoot through state incorporates to bring down exchanging misfortunes than PWM voltage source inverter due to added on-off activity of switches in ZSI rummage-sale to add the shoot through periods. Due to this reality notwithstanding decrease the cost, volume and weight, in the proposed strategy ZSI inductor chose minuscule so as the ZSI arrives in to the third activity mode. The grid associated PV framework with ZSI ought to be concentrated to manage power in network side distribution framework. The proposed distribution regulator measure is introduced in beneath segment.

\section{Proposed Distributed Control structure}


The proposed distributed control structure of grid connected PV system is explained in this section. In the section, the control design characteristics are analyzed and specified in the figure 4 . The control process of grid connected PV system is used to reduce the voltage and current fluctuations in proposed architecture. The PV system is generated power based on different irradiance condition, mostly irradiance is fixed as $1000 \mathrm{WW} / \mathrm{m}^{2}$ which generates maximum power to compensate heavy load request in grid side. In our proposed system is concentrates rooftop placement of each home which avoid extra placement of PV panel system. Inverter three phase output voltage is formulated in the equation (8)

$$
\left[\begin{array}{l}
V_{a} \\
V_{b} \\
V_{c}
\end{array}\right]=\left[\begin{array}{c}
V_{m} \cos (\omega t) \\
V_{m} \cos \left(\omega t-\frac{2 \pi}{3}\right) \\
V_{m} \cos \left(\omega t+\frac{2 \pi}{3}\right)
\end{array}\right]
$$

Inverter three phase current is presented in equation (9)

$$
\left[\begin{array}{l}
I_{a} \\
I_{b} \\
I_{c}
\end{array}\right]=\left[\begin{array}{c}
I_{m} \cos (\omega t) \\
I_{m} \cos \left(\omega t-\frac{2 \pi}{3}\right) \\
I_{m} \cos \left(\omega t+\frac{2 \pi}{3}\right)
\end{array}\right]
$$

Where, $I_{m}$ is the maximum current, $\omega$ is the angular frequency and $V_{m}$ is the maximum voltage of $V$. The calculated three phase inverter current as well as voltage is changed in to two axis rotating coordinated with the computation of park transformation. The park transformation with convection of voltage and current is formulated below equation (10).

$$
\left[\begin{array}{l}
V_{\alpha} \\
V_{\beta}
\end{array}\right]=V_{a b c \rightarrow \alpha \beta}\left[\begin{array}{l}
V_{a} \\
V_{b} \\
V_{c}
\end{array}\right]=\left[\begin{array}{l}
V_{m} \\
0
\end{array}\right]
$$

Where, $\alpha-\beta$ and $V_{\alpha}$ and $V_{\beta}$ is considered as the coordinate system. Similarly, the parks transformation of the inverter is presented in follows, 


$$
\begin{gathered}
i_{\alpha-r e f}=\frac{P_{r e f}}{V_{\alpha}} \\
i_{\beta-r e f}=\frac{Q_{r e f}}{V_{\beta}}
\end{gathered}
$$

Using this inverter yields, power estimation can be refined. In power calculation, reactive in addition real power can be determined. Using reactive and real power references regards are used towards register the error worth in addition change in error esteem. In the control structure of the disseminated is obviously portrayed in figure 5 .

With the consumption of, $P_{r e f}$ and $Q_{r e f}, P$ and $Q$ are compared, and error standards computed. Generally, the real in addition reactive power is used to compute the voltage amplitude $(E)$ in addition frequency $(\omega)$ by using various conditions. The frequency and amplitude computations are reduced by using ESO and PSO algorithms. Prior to that, expectant the two circumstances for $\mathrm{P}$ in addition $\mathrm{Q}$ control,

1. Real power $(\mathrm{P})$ basically relies upon converter voltage point, so that is varying by $\omega$.

\section{Based on voltage amplitude ( $E$ ), Reactive power Q is computed.}

From the above two points, the distributed control of MG is achieved with the proposed controller. The frequency and voltage amplitude calculations are given below,

$$
\begin{gathered}
\omega=\omega^{*}-S_{\alpha}(s) \cdot\left(p-p^{*}\right) \\
E=E^{*}-S_{\beta}(s) \cdot\left(q-q^{*}\right)
\end{gathered}
$$

Where, $S_{\alpha}(s)$ and $S_{\beta}(s)$ corresponding transfer functions, $p^{*}$ and $q^{*}$ can be referred value of real in addition reactive power, $E$ is the referred voltage amplitude, $p$ and $q$ is the calculated reactive and real power, $\omega^{*}$ is the nominal frequency, $E^{*}$ can be the nominal voltage amplitude, and $\omega$ is the referred frequency. 


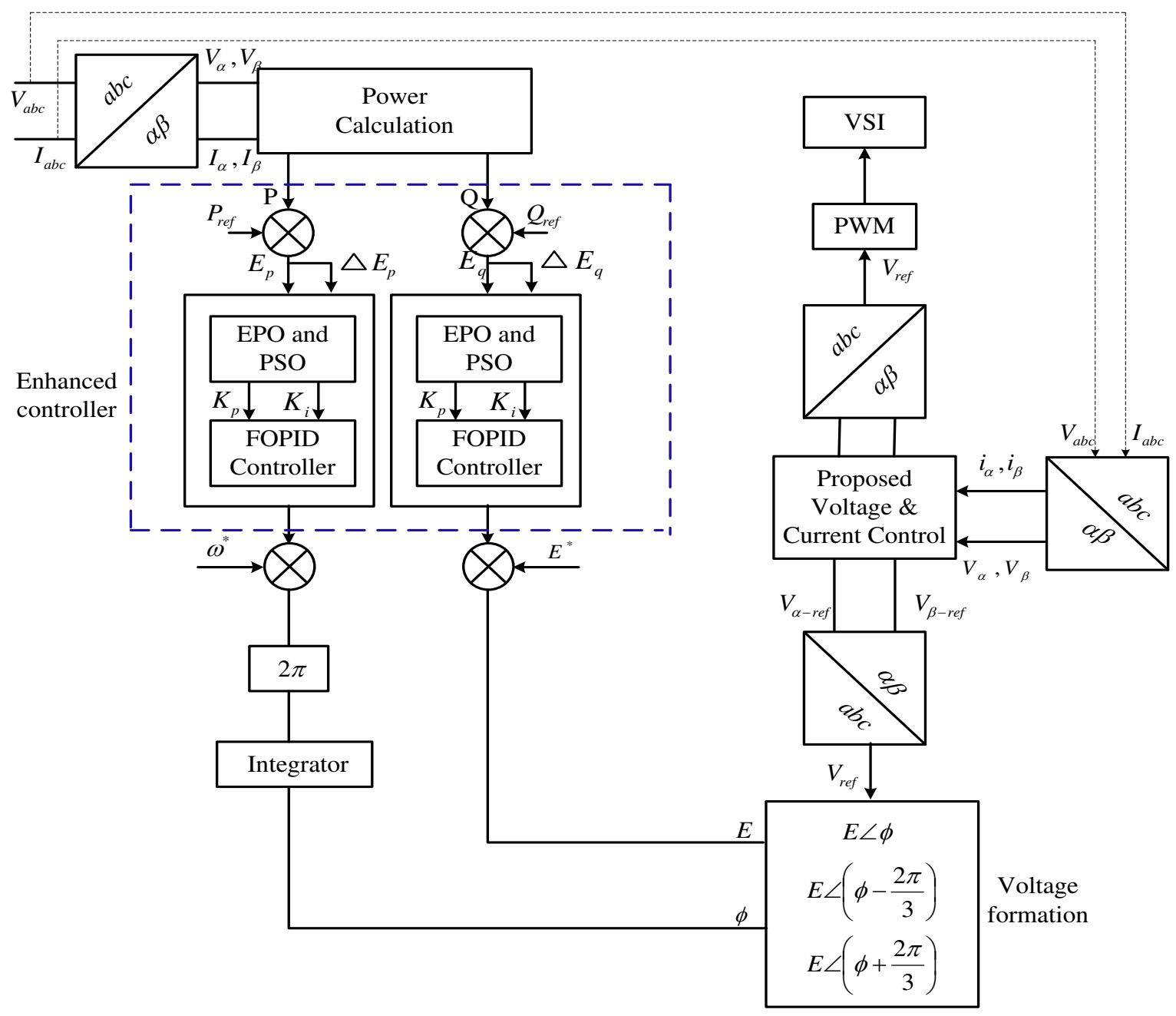

Figure. 5 Control schemes of proposed method with FOPID controller

In the proposed control structure, $S_{\alpha}(s)$ and $S_{\beta}(s)$ are considered as constant distributed coefficients. The constant value can be calculated by (8) and (9).

$$
\begin{aligned}
& S_{\alpha}(s)=\frac{\Delta \omega_{\max }}{P_{\max }}=s \\
& S_{\beta}(s)=\frac{\Delta E_{\max }}{Q_{\max }}=t
\end{aligned}
$$

Where, $\Delta E_{\max }$ is the maximum allowed voltage frequency, $\Delta \omega_{\max }$ is the maximum allowed voltage amplitude distributed, $t$ is the reactive power coefficient, $s$ is the real power coefficient, 
$P_{\max }$ is maximum allowed real power, and maximum allowed reactive power is denoted by $Q_{\max }$. After that, two different values are computed which voltage formation in addition voltage amplitude. Once completed the voltage formation computation $V_{\text {ref }}$ voltage, it can be computed from three phase voltage to two axis range $\alpha-\beta$ axis especially for current as well as voltage through the park transformation procedure. In the proposed technique, the actual value and the variation in the values of the voltage and current are evaluated, which are furnished as the input of the FOPID-EPO with PSO technique. Here, the FOPID performance is enhanced using the EPO with PSO technique. With the utilization of proposed technique, the performance of FOPID controller is enhanced. To enhance the performance of the FOPID, the optimal gain parameter is determined. The controller is evaluated, and optimal parameters computed. The improved performance of the FOPID controller is presented in section 4.1.

\subsection{Design of FOPID controller}

The FOPID regulator depends differentiation, proportional in addition of integral order. A fractional order differential condition can be used towards portray the fractional order regulator. In this controller, three boundaries $\mathrm{Kp}, \mathrm{Ki}$ and $\mathrm{Kd}$ ought to be adjusted to plan regulator [27]. Most potential outcomes to enhance PID regulator is to use fractional order controllers. And, distinction among FOPID and PID regulator is that, in FOPID, the request for subsidiary and vital isn't number. This symbol provides additional levels of chance in alteration the regulator and quick better powerful implementation in examination with customary PID Due to expanding interest in improving the reaction of traditional PID, FOPID regulator has gotten significant consideration over the most recent couple of year. The yield of FOPID regulator as far as time space is spoken to as follow,

$$
\begin{gathered}
u(t)=k_{p} e(t)+k_{i} D_{t}^{-\lambda} e(t)+k_{d} D_{t}^{\mu} e(t) \\
e(t)=\Delta P_{\text {tie }}(t)+B_{i} \Delta \omega_{i}(t)
\end{gathered}
$$

FOPID controller transfer function is computed as follows, 


$$
G(s)=k_{p}+\frac{k_{i}}{s^{\lambda}}+k_{d} s^{\mu}
$$

Where e(t) can be described as error parameter which computes with desired set point and output variable. The output controller can be computed based on FOPID controller gains. Different PID controller parameters tuning techniques are utilized for decentralized power system load frequency controller. The worldwide advancement procedure like EPO with PSO has concerned the thought in the field of controller parameter improvements. In this paper, EPO with PSO is proposed to produce optimal pulses to ZSI which reduce the oscillations among the grid connected PV system in distributed systems.

\subsection{Proposed Hybrid optimization algorithm}

The grid connected PV system with control structure is enhanced by selecting optimal pulses of ZSI with the help of optimization algorithm. The optimization algorithm is combined by two different algorithms such as EPO and PSO algorithm. The updating process of EPO algorithm is attained with the help of PSO algorithm. The process of individual grid connected PV system is explained follows,

\subsubsection{Emperor penguin optimization}

The distributed controller of grid connected PV system can be attained with the help of the EPO with PSO. The optimal pulses of ZSI converter is generated based on the error values current, voltage, real and reactive power of grid connected PV system. The mathematical formulation of the EPO is presented in below section.

\subsubsection{Inspiration of the EPO}

The EPO algorithm is developed based on the behavior of emperor penguin that methodically called as Aptenodytes forsteri in addition it weightiest from the penguin classes. From plumage in addition size, the female and male penguins are very much similar. The penguin has the black color dorsal side and head [28]. Similarly, penguin have the white color belly, yellow color breast and bright yellow color patches. In the winter seasons, the emperor penguins devote their, survives 
in exposed size in addition types. In the breeding periods, the penguins are coming ashore in large crowds that contains hundreds of thousands of penguins. The female penguin can be travel to 50 miles to reach ocean for getting prey even lay a single egg. The hunting behavior and foraging behavior based, emperor penguin named as an animal in a group. Additionally, the emperor penguin has the ability to dump up to 1900 bases profounder in addition live below sea within 25 minutes. It can be stiffened and flattened wings with other penguin's species. To live the glacial season condition, the emperor penguins have the huddles. The huddling characteristics of emperor penguins are developed into four stages that presented below,
* Develop and compute the of emperor penguins of huddle boundary
* Compute temperature outline surrounding the huddle
* compute the coldness among emperor penguins
* Emperor penguin can be relocating the effective mover

\subsubsection{Mathematical modelling of EPO}

The emperor penguins are working based on the huddling behavior that mathematical representation is presented in the section. The main objective of the emperor penguin is to compute out best mover of penguin. In behavior, huddle can be expected to be presented on two different dimensional L-shape polygon planes. Initially, penguin create random huddle boundary. However, the profile of temperature related the huddle can be calculated. The aloofness among emperor penguins can computed that will be useful aimed at exploitation and exploration. At last, the best mover is called as the optimal answer which can be taken as the optimal pulses to tune the parameters to solve the distributed controller. The best solution can be achieved and recalculate the huddle boundary conditions with updated positions of emperor penguins. The update positions of the process in the EPO is presented in the below section.

\section{Create and compute huddle boundary}

The emperor penguin generally updates the position themselves with the shape of polygon grid boundary under the huddling stage. In the huddling process, the emperor penguins can at low two neighbors that can be selected arbitrarily in the huddling procedure. In huddle behavior, wind flow about huddle can be computed to discovery out the huddle border related a polygon. Moreover, the wind flow of the emperor penguin can be greater than the program of a penguin [29]. To 
describe the arbitrarily created huddle boundary, concepts of complex variables in emperor penguin. The wind velocity $\alpha$ in addition, $\beta$ can be defined as the gradient of $\emptyset$, which mathematically described in the below equation,

$$
\beta=\nabla \emptyset
$$

Where, $\delta$ can be mixed with $\emptyset$ to create the complex potential which can be described as the below equation,

$$
S=\varnothing+j \delta
$$

Where, $S$ can be described as the analytical function and $j$ can be described as the imaginary constant. The two-dimensional environment of the emperor penguins is described in the equation (14). Generally, the penguins are update position based on their current position of emperor penguin that is to presented as centered of polygon region with L-shaped under greater best fitness rate under iteration procedure.

\section{Temperature profile regarding the huddle}

Generally, the emperor penguins create group to increase the ambient temperature and conserve energy in the huddle. The situation can be mathematically modelled with the utilization of different assumptions that temperature is zero condition when radius of polygon can be less than one and temperature zero condition, the radius of polygon can be greater than one. Based on the exploration and exploitation procedure in the emperor penguins, the temperature profile is changed under different locations [26]. Under the huddle conditions, the temperature profile can be represented as below,

$$
\begin{aligned}
& T_{C}=\left[T-\frac{\text { MaX Iteration }}{Y-\text { MaX Iteration }}\right] \\
& T= \begin{cases}0, & \text { if } P>1 \\
1 & \text { if } P<1\end{cases}
\end{aligned}
$$

Where, $T$ can be represented as time for computing best optimal results in search space, $P$ can be represented as radius of polygon, $Y$ can be represented as the present iteration and MaX Iteration can be represented as the maximum number of iterations. 


\section{Distance among emperor penguins}

The distance among best attained optimal solution and emperor penguin can be calculated after the creation of boundary in huddle. When developed solution is close to the fitness solution that can be defined as the optimal solution. The remaining penguins (search agents) would be apprise their positions related to present best optimal solution that are formulation distinct as follows,

$$
\vec{d}=A B S\left(s(\vec{a}) \cdot \overrightarrow{r(Y)}-\vec{c} \cdot \overrightarrow{r^{s}(Y)}\right)
$$

Where, $\vec{a}$ and $\vec{c}$ can be coefficients that used for avoiding collision among neighbors, $\vec{d}$ can be represented as the distance among best fitness search agent and emperor penguin, $\overrightarrow{r^{s}}$ can be represented position vector of emperor penguins, $\vec{Y}$ can be represented as the current iteration, $\vec{r}$ can be represented as the best optimal pulses and $s(\vec{a})$ can be represented as the social forces of emperor penguins which is responsible to move towards the path of best optimal best solution that best search agents. The coefficient vectors are calculated based on the below equation,

$$
\begin{gathered}
\vec{a}=\left(m \times\left(T+r^{\text {grid }}(A C C)\right) \times \text { Random }(\quad)\right)-T \\
r^{\text {grid }}(A C C)=A B S\left(\vec{r}-\overrightarrow{r^{s}}\right) \\
\vec{c}=\text { Random }(\quad)
\end{gathered}
$$

Where, Random ( ) can be represented as the random function presented in the value of $[0,1]$, $r^{\text {grid }}(A C C)$ can be represented as the accuracy of polygon grid through contrasting the change among emperor penguins, $m$ can be represented as movement component which keeps a gap among search agents aimed at avoid crash, parameter is set as the value 2, $T$ can be represented as huddle based temperature profile. And, function of the $s()$ can be computed in the below equation,

$$
s(\vec{a})=\left(\sqrt{F \cdot e^{-\frac{y}{L}}-e^{-Y}}\right)^{2}
$$

Where, $\mathrm{F}$ and $\mathrm{L}$ can be represented as control parameters for best exploitation and exploration that values the range of $[2,3]$. The proposed EPO algorithm delivers optimal results among this ranges.

\section{Relocate the mover}


The emperor penguins positions can be updated related to the best optimal solutions that is called as the mover. The changing position of the penguin is attained based on the mover responsible of different search agents in a presented vacated and search space the next position that one presented position. And, updating process of the next position of an emperor penguin are presented in the below equations,

$$
\overrightarrow{r^{s}}(Y+1)=\overrightarrow{r(Y)}-\vec{a} \cdot \vec{d}
$$

Where, $\overrightarrow{r^{s}}(Y+1)$ can be represented as the emperor penguin new updated positions. In the repetition procedure, the penguin bunching characteristics is recomputed after the agent has been relocated. The proposed EPO algorithm can be used to finding optimal pulses of the DC-DC converter and ZSI for grid connected PV system. The proposed process of the proposed method is presented in the below sections.

\subsection{Particle swarm optimization}

The innovative PSO technique exploits the quicker calculation duration of the PSO in tandem.

To update the positions of the penguins, particle algorithm is used in this paper. The detail explanation of the PSO is presented in below section.

Step1: Initialize position $p_{i}$ and Velocity $v_{i}$ discretely and roosters current position.

The PSO activates the population which is a throng of expressions offered by the client. The PSO technique commences with activation of the population [30], which embraces the input data. Hence, the locality of particle i characterized as:

$$
X_{i}=\left(x_{1}, x_{2}, \ldots \ldots x_{n}\right)
$$

Where, $X_{i}$ - locality of the particle.

The particle encompasses memory of the prior best position, expressed by the relation below:

$$
P_{i}=\left(p_{1}, p_{2}, \ldots \ldots . p_{n}\right)
$$


Where, $p_{i}$ - prior best position

The velocity of the particle is furnished as per the expression:

$$
V_{i}=\left(v_{1}, v_{2}, \ldots \ldots v_{n}\right)
$$

Where, $V_{i}$-Velocity of the particle

Step2: In respect of the entire particle calculate fitness by means of the relative equation.

The penguin positions are updated with the help of particle algorithm. So, the position of penguin's updating is fixed as a fitness function.

$$
F=\operatorname{Update}(P(\text { Penguins }))
$$

The penguin position updating process is achieved with the help of PSO algorithm. Based on the global best conditions, the greater fitness function is achieved.

Step 3: If fitness is better in relation to the earlier $\mathrm{p}$ current values in best set as the optimal p best. The fresh populations can be estimated as per the two stipulations stipulated by the PSO.

$$
v_{i}^{(n+1)}>v_{m}^{(n+1)} \text {, then } v_{i}^{(n+1)}=v_{m}^{(n+1)}
$$

Step4: Continue to perform step3 and step4 for the whole particles in addition choose the optimal particle as the $\mathrm{g}$ best.

The characteristics are velocity and position of the components in the population, and in the existing circumstances, the vocabularies are deemed as the parameters of the population. In addition, each particle has apt data about the best value till now in the group ( $\mathrm{g}$ best) between $\mathrm{p}$ bests. This data can be akin to the comprehension of the way the other particles in the vicinity have accomplished themselves. Each particle shows the tendency to vary its location by means of the ensuing data such as the distance among the best position and current position, the distance 
between the current position and $\mathrm{g}$ best. The updating of the function is made likely by means of the equations shown below:

$$
\begin{gathered}
\text { velocity }=\text { velocity }^{c}+\alpha(\text { pbest }- \text { pos } \\
\end{gathered}
$$

Step 5: Perform for the entire particle calculate the velocity and revise position

Every particle is gifted with a best portion called pbest, if refreshed position is higher than the pbest, at that time it is deemed as the pbest. The optimal parameter amongst the pbest is taken as the gbest parameter of the entire population. Uncertainty the revised position exceeds gbest, it can represent as the gbest. The procedure gets replicated till a stage arrives, in which the termination benchmarks are satisfied, iterations being habitually deemed as the termination standards.

Step6: If the maximum iteration is not met, proceed to continue steps coming after the fitness function step. Using the proposed algorithm, the optimization method is achieved. The EPO algorithm updating procedure is attained with the assistance of the PSO algorithm. The process of proposed algorithm EPO with PSO is explained in this phase. The steps of projected hybrid algorithm is presented in below section.

\subsection{Process of EPO with PSO distributed controller in PV}

The proposed EPO algorithm is used to select the optimal pulses to the ZSI for reducing the error of the grid connected PV system. The optimal pulses are generated based on the error of the grid connected PV system. The PV generation system is mainly used for providing power supply to the PV system. With the use of the projected EPO with PSO algorithm, the error value minimized and improve the performance of the PV system. The ZSI converter is used to reduction of the error for stabilizing the PV operation and enhance the presentation of the system, the projected EPO-PSO 
algorithm process can be illustrated in figure 6 and explanation of the proposed EPO- PSO algorithm is presented below,

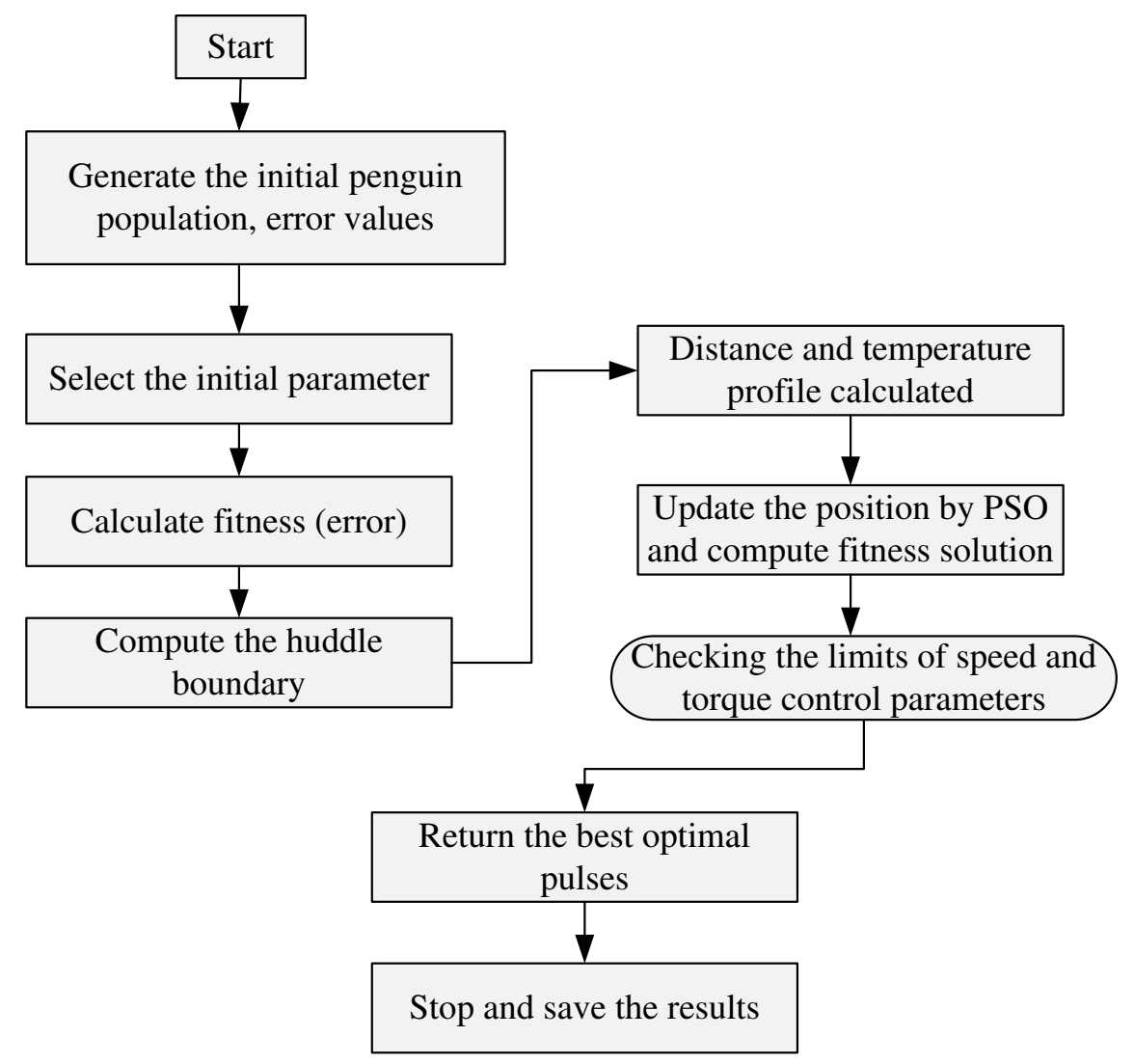

Figure. 6 EPO algorithm flowchart

Step 1: Population initialization progress, control parameters of error values of grid connected PV system.

Step 2: Select the initial parameters constraint limitations of PV system and maximum iteration

Step 3: Compute the fitness value, in this proposed EPO algorithm minimization of voltage and current error values are taken as the fitness function.

Step 4: Compute the boundary of huddle conditions of the emperor penguin behaviors. 
Step 5: Related on the huddle behavior, the temperature profile can be computed to attain the optimal pulses of the ZSI.

Step 6: Calculate the distance among the emperor penguins for update the positions of the penguins to select the best search agents which attained with the assistance of PSO algorithm.

Step 7: Based on the distance and PSO algorithm, the position of the penguins are updated.

Step 8: The boundary conditions of the emperor penguins and PV system with FOPID controller constraints are checked

Step 9: Compute update the position and update search agent fitness value of lastly attained optimal pulses of ZSI.

Step 10: The algorithm is stationary when it reached the maximum iteration and optimal results are achieved.

Step 11: Finally, best optimal pulses of ZSI is find out which are feed to the ZSI, it reduced the error values of grid connected PV system.

Finally, with consumption of the projected EPO-PSO algorithm, the optimal pulsed of the ZSI is computed based on the fitness function. The optimal pulses are able to reduce the error values of PV system and it is stabilizing the system. The presentation of the projected technique is validated with the results section. The performance evaluation is essential to demonstrate the efficiency of the proposed technique. The experimental outcomes of the projected technique is presented in the following section.

\section{Performance evaluation}

In this segment, presentation of the projected coordinated strategy implemented in MATLAB/SIMULINK working phase is depicted. To dissect the projected mixture control method, PV framework is utilized. Figure 7 shows the PV framework associated with the proposed control strategy. The presentation investigation of the intensity of the PV is broke down and shown in figure 8 . As indicated by the variety of the sun-oriented irradiance, the most extreme yield power is followed. The generated power is normally given to the nonlinear load through methods for the 
transmission lines. And, the control pulse creation of the converter can be gotten with using projected strategy, that depends on the current and voltage of PV board.

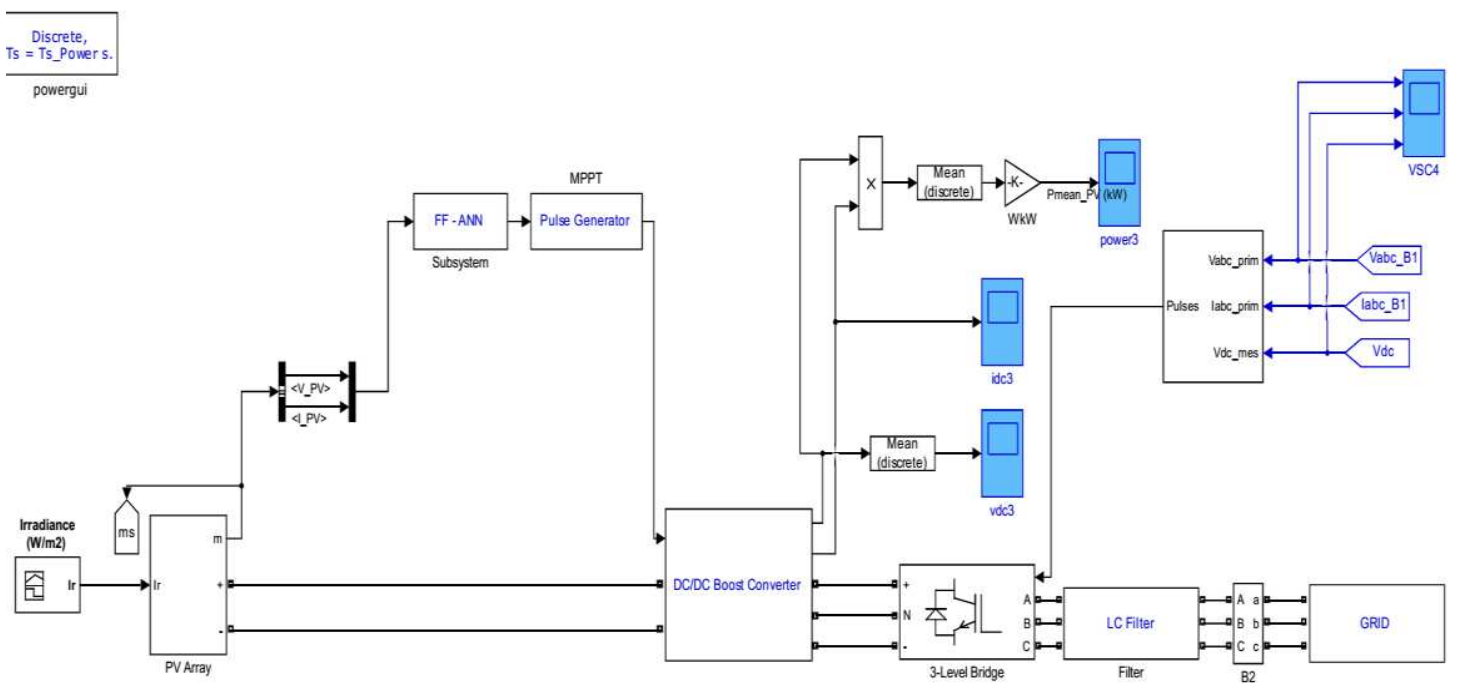

Figure. 7 Proposed control system in MATLAB/Simulink

The projected technique finely utilizes the current and voltage assets as the info. In occasion the methodology reaches a conclusion, the EPO with PSO turns out to be very much outfitted to produce the optimal switch pulses of the converter. The grid connected PV system performance can be improved with the proposed controller. The MPPT controller can be mainly focused related to SEPIC converter and grid side controller is focused related to ZSI inverter. To analysis the performance of grid connected PV system is analyzed with two various scenario, constant irradiance condition and ramp irradiance condition.

\subsection{Performance analysis}

In section, the proposed method performances were evaluated and discussed. The implementation results were analyzed with change irradiance condition at the time seconds $t=0$ to 0.25 seconds. The solar irradiance is analyzed in this section.

Case 1: Analysis of Constant irradiance

Case 2: Analysis of Changed irradiance

\section{Case 1: Analysis of constant irradiance}


For the situation, the sustainable power assets are steady that speaks to the irradiance of PV is thought to be $\left(1000 \mathrm{~W} / \mathrm{m}^{2}\right)$. For the visual portrayal, the irradiance is portrayed in the figure 8 . In view of the examination, the produced intensity of PV is estimated and delineated in the figure 10. The rough created intensity of PV can be $2900 \mathrm{~W}$. For situation, the load can be different, and the load demand will be thought to be $6000 \mathrm{~W}, 8000 \mathrm{~W}, 12000 \mathrm{~W}$ separately. In the power management conditions, the all termination condition should be checked it should be compensating load demand. At that point just, the optimal power flow is accomplished. In this way, the energy storage devices are thought of, similar to, battery. In the paper, the storage device of battery is used to redress in addition fulfilled the load demand, for the investigation cycle, the discharging and charging cycle is likewise done in the figure 11. For the most part, the battery is associated for capacity reason. At the point when the load demand isn't remunerated with the PV power, the battery is attempting to satisfy the need. The ZSI execution are broke down and represented in figure 9.

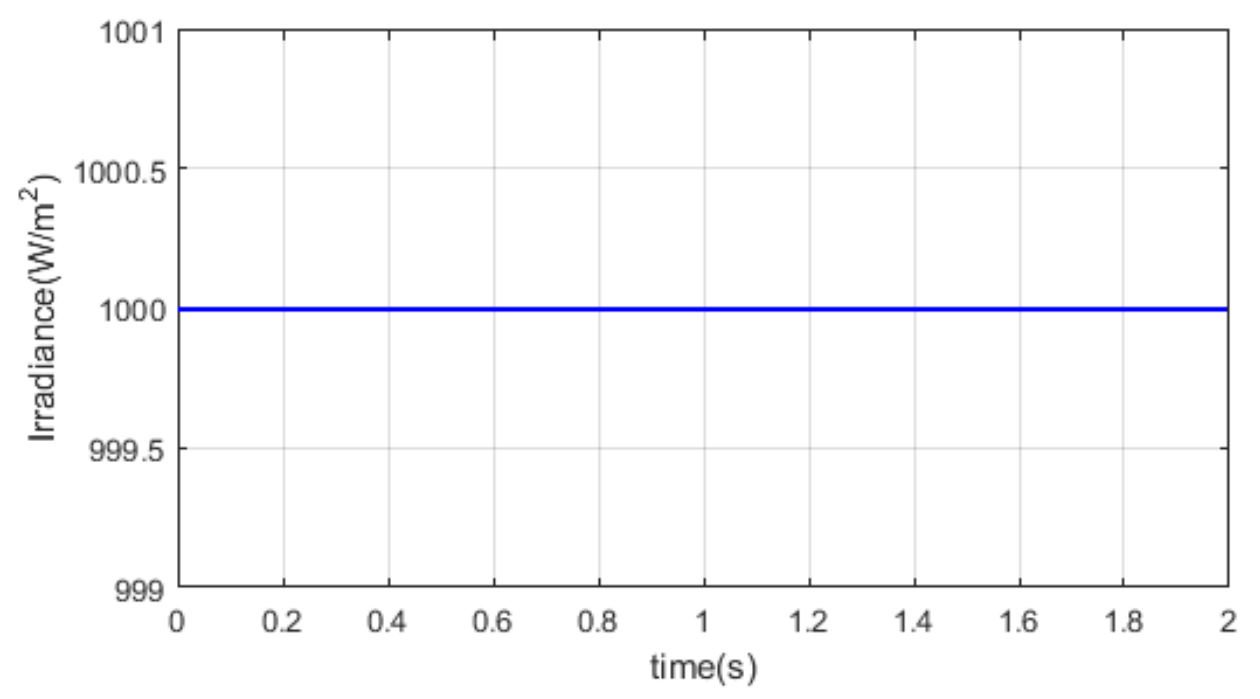

Figure. 8 Analysis of Irradiance level 


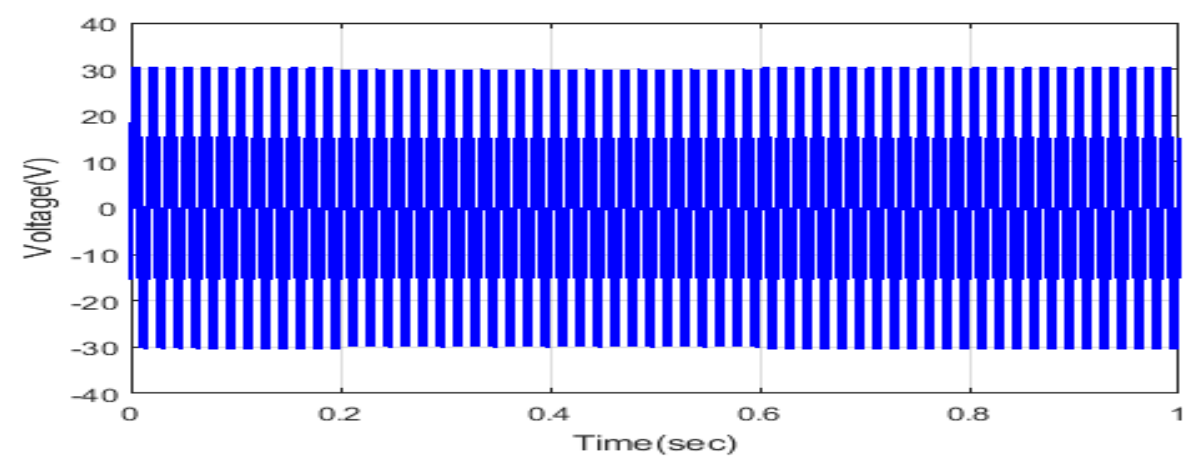

(a)

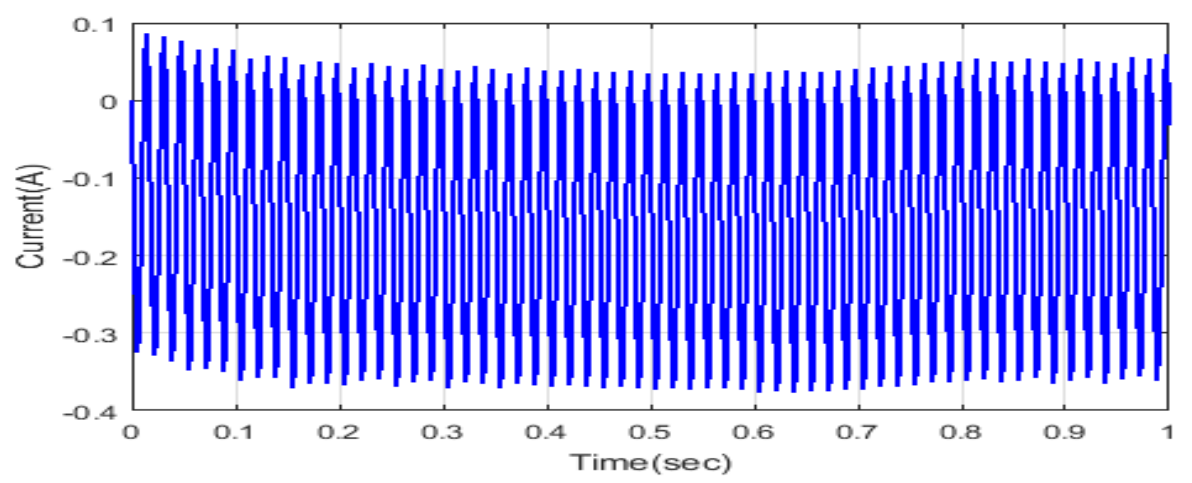

(b)

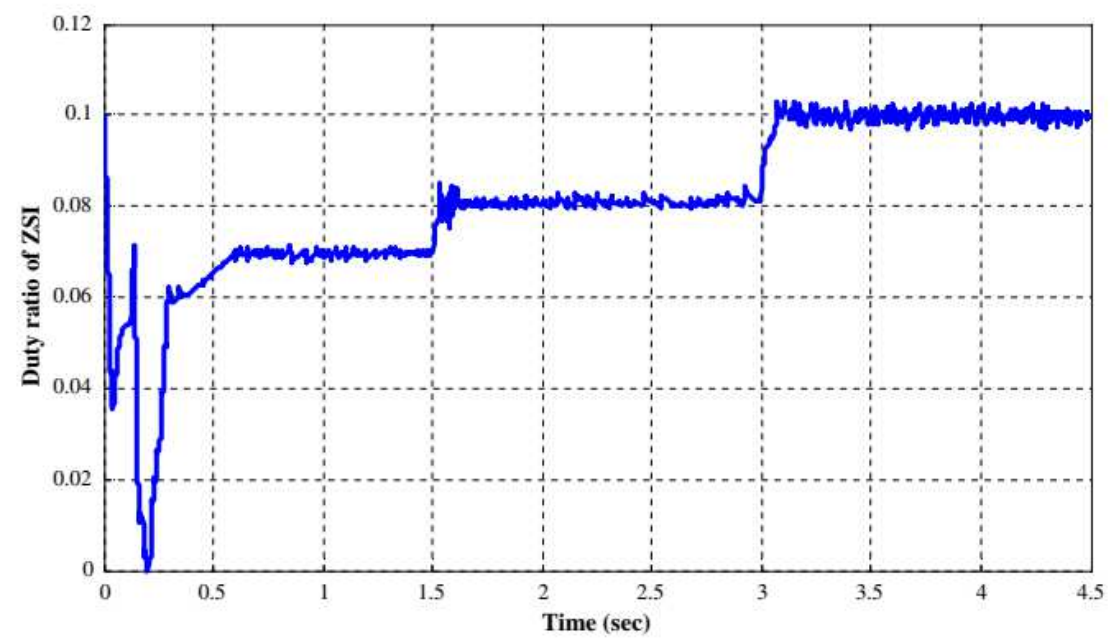

(c) 


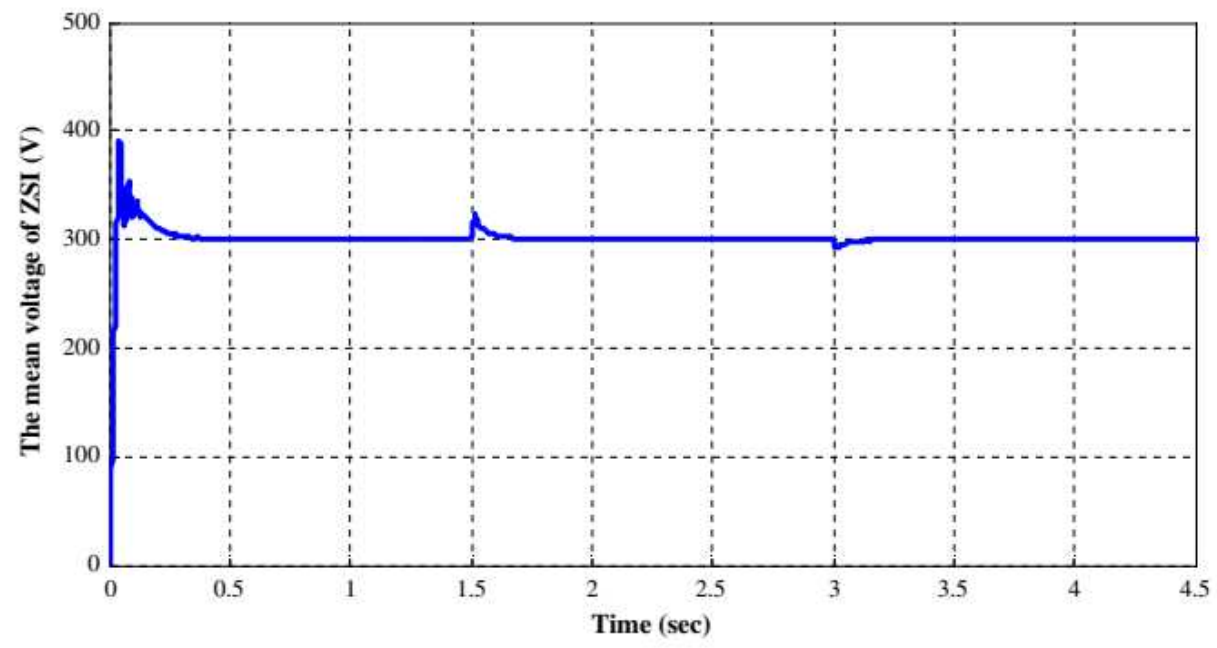

(d)

Figure. 9 Analysis of (a) output voltage of SEPIC converter, (b) current of SEPIC converter, (c) Mean voltage of ZSI and (d) Duty ratio of ZSI using proposed method

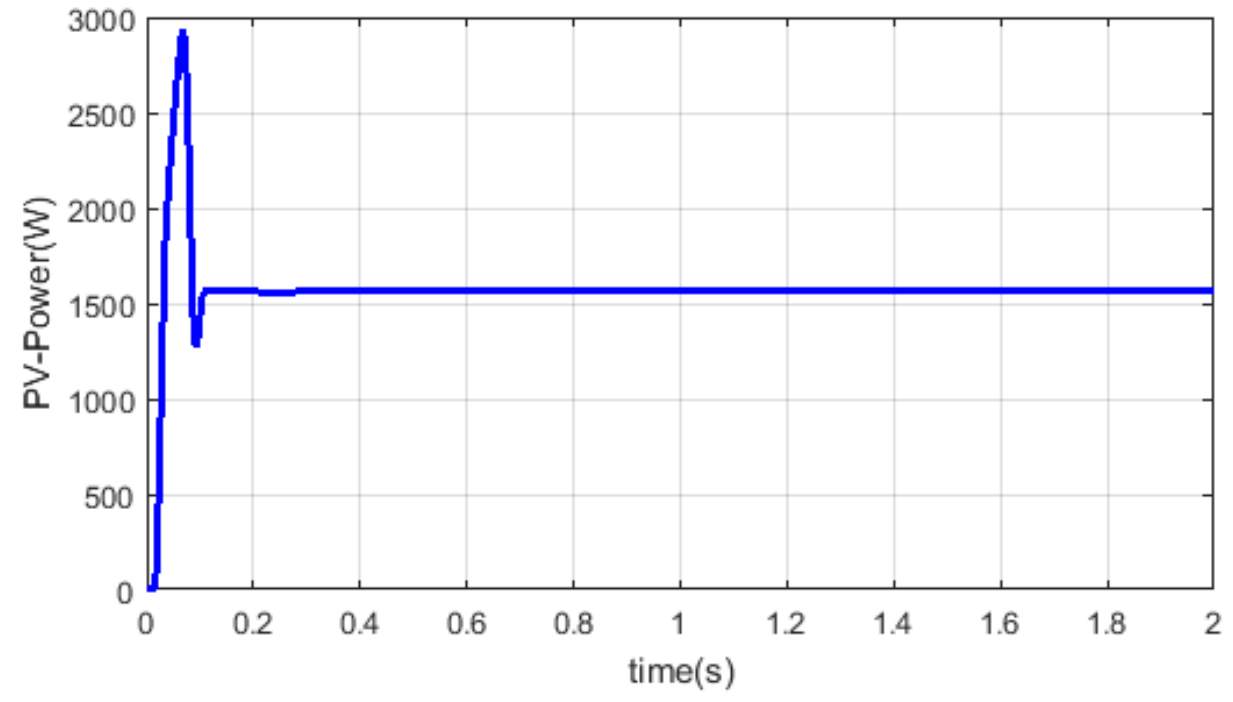

Figure. 10 Analysis of PV generated power 


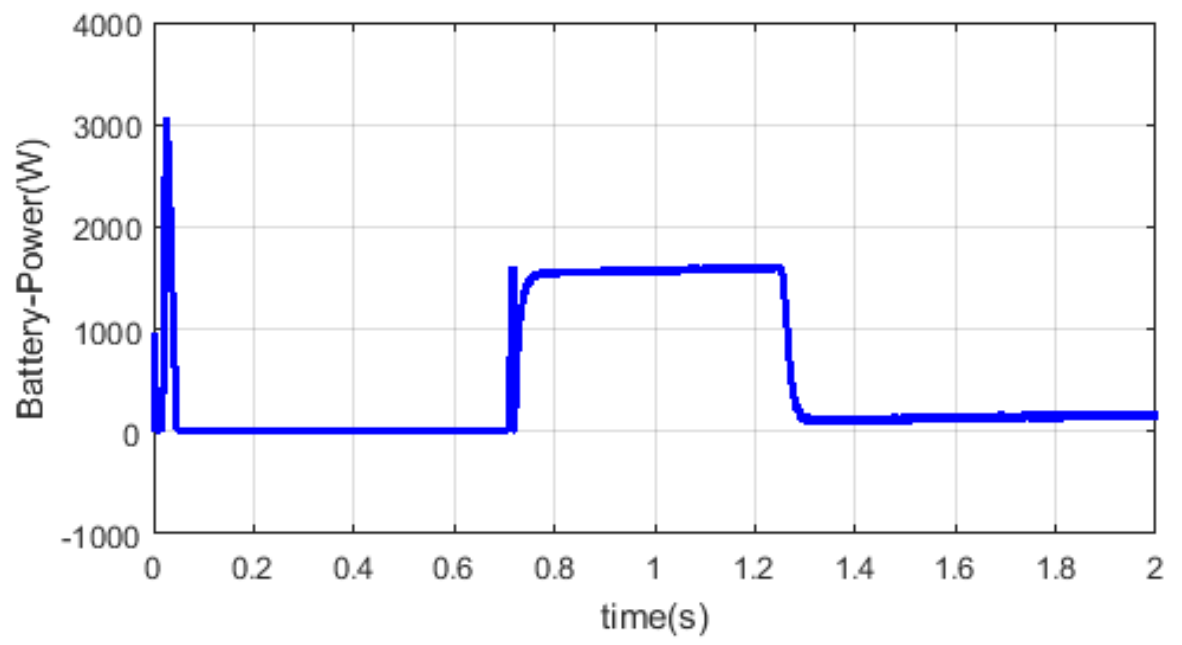

Figure. 11 Analysis of battery charging power

In figure 11, at starting point, battery is charging mode of operation in the condition of $0.1 \mathrm{~s}$ to $0.75 \mathrm{~s}$. After that, the maximum power is attained to $1500 \mathrm{~W}$ at the time interval of $0.75 \mathrm{~s}$ to $1.3 \mathrm{~s}$. The battery is providing the generated power to the grid side for compensating load demand. Due to the demand variation, the generated power as well as grid power is analyzed. The illustration of the generated and grid side power is given in figure 12 .

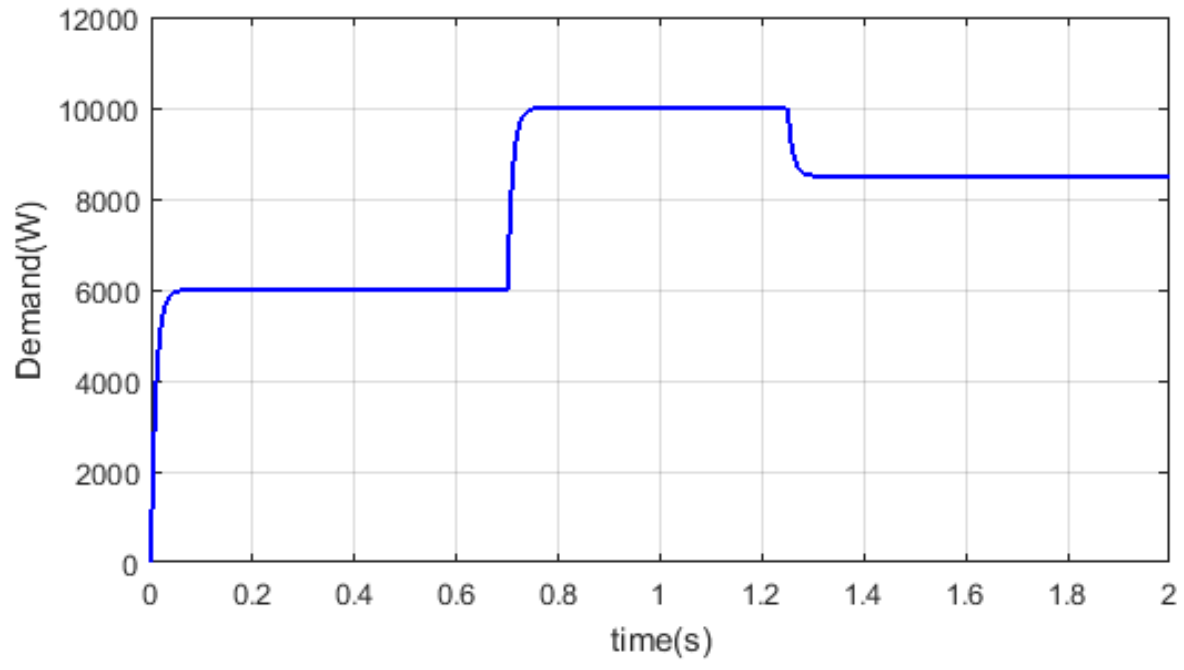

(a) 


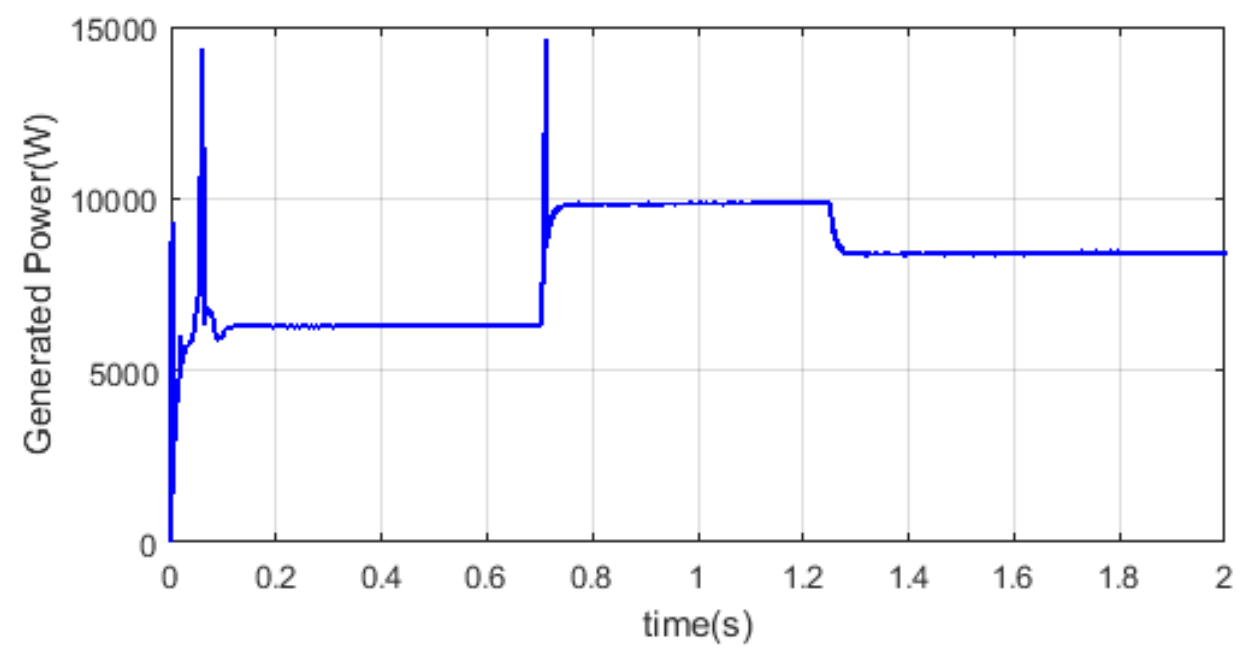

(b)

Figure. 12 (a) Load Demand and (b) Generation power

For this situation the load demand variety can be taken at the diverse time moment. At first, the time moment $\mathrm{t}=0-0.7 \mathrm{sec}$, load demand can be thought to be $6000 \mathrm{~W}$ in addition the generated power from the info sources are broke down simultaneously moment. Considering the investigation, the force is most extreme accomplished and fulfills the load requirements. From that point onward, whenever span $\mathrm{t}=0.7-1.3 \mathrm{sec}$, interest can thought to be $10000 \mathrm{~W}$ and assessed produced power from the inexhaustible assets in addition energy storage devices are likewise fulfill the load requirements which is spoken to in the figure 12(b). Additionally, the load demand is shifted after the specific time moment, and afterward expected the forces. To satisfy the load request, yet again the generated power can be assessed. To achieve optimal operation of intensity the executives, the management control is created. At the point when the load demand power can be expanded, the battery is giving the additional capacity to the framework for remunerating the required power in the network associated PV framework. For instance, the generated power is $5500 \mathrm{~W}$ at the hour of 0 s to $0.7 \mathrm{~s}$ and which time load demand can be $6000 \mathrm{~W}$. The PV power should be satisfying need, however the interest isn't to meet methods, necessary power can be produced from the battery. Assume, the abundance power is created from the data sources implies, that is put away from the energy storage devices. The load demand management is operating dependent on the control of 
reactive and real power. Investigation of accomplished output network power can be spoken to the figure 13.

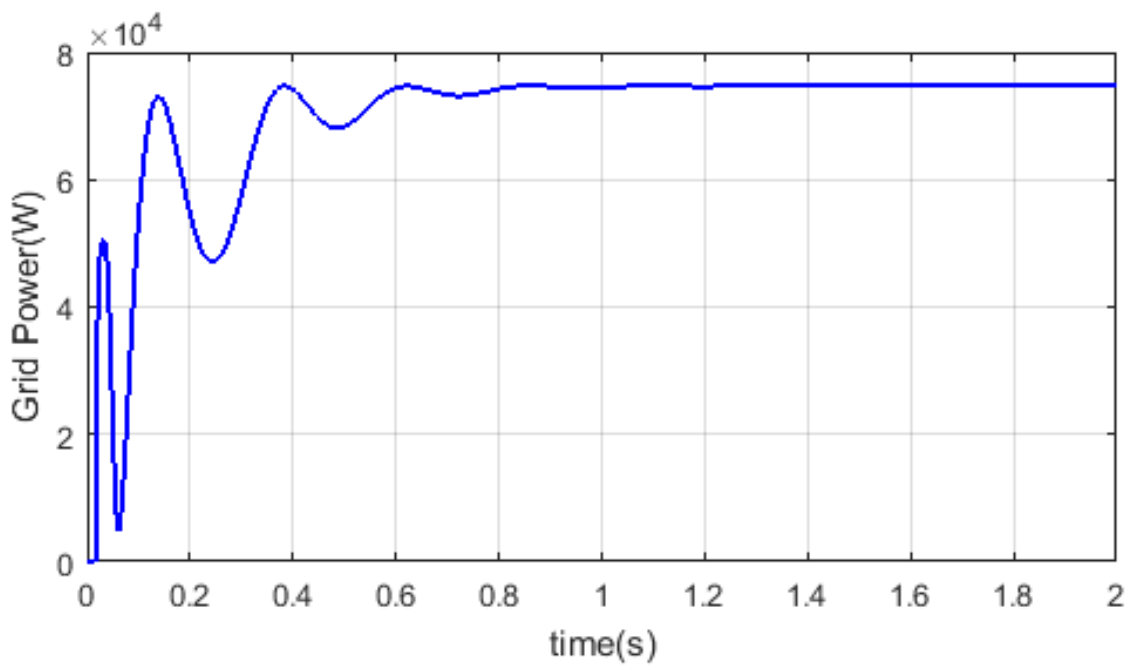

Figure. 13 Analysis of the output grid power

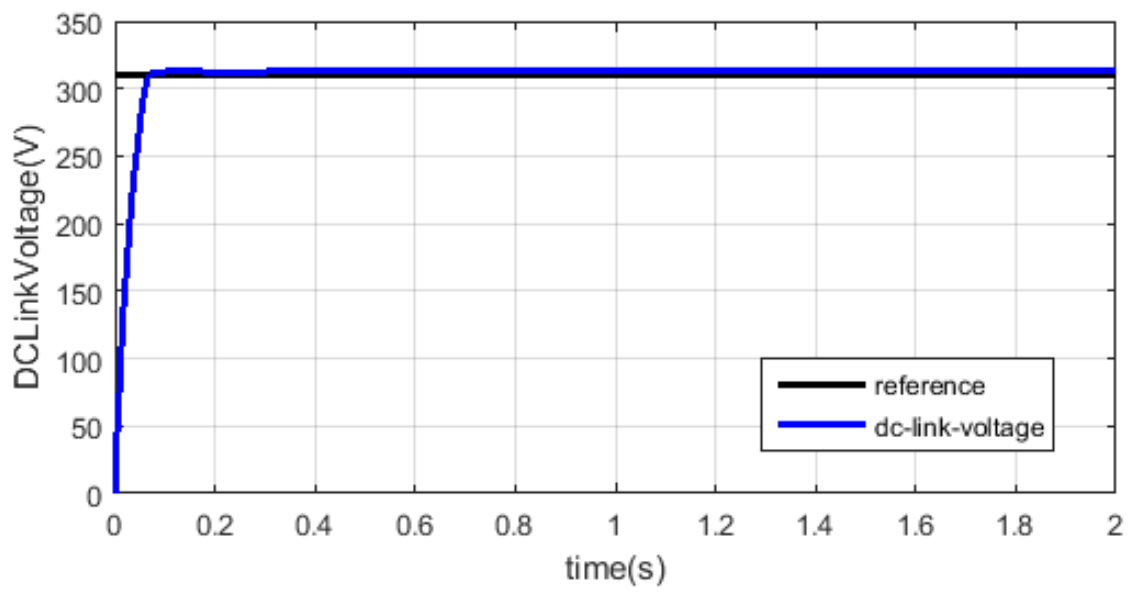

Figure. 14 Dc link voltage Analysis

In proposed distributed control, reactive and real power management is finished. Moreover, the voltage and current control circles are created through using the proposed calculation. For the voltage guideline circle, the improved FOPID regulator provides the dc link voltage at constant level. In current regulator circle, the maximum current can be extricated by using the proposed controller. To achieve the optimal tuning procedure, the regulator gain boundaries can be 
adequately achieved and created the best outcomes. Presentation examination of dc connect voltage is depicted in this figure 10 at the time moment $t=0-2 \mathrm{sec}$. All things considered, the reference voltage is $310 \mathrm{~V}$ in addition need to keep up the dc link voltage as steady with the reference voltage. At first, the dc link voltage is $0 \mathrm{~V}$ at the time moment $0-0.1 \mathrm{sec}$ after that it increments with equivalent to the reference voltage. From that point onward, the dc link voltage is proceeding onward with the steady condition. Utilizing this dc link voltage, examination investigation is accomplished in this case it is introduced in the following area 4.2. Also, the case 2 exhibitions are dissected and portrayed in the accompanying,

\section{Case 2: Analysis of various irradiance}

For this situation, presentation investigation PV irradiance variety is portrayed in the figure 15 (a) and the irradiance of PV can be thought to changes from $1000 \mathrm{w} / \mathrm{m}^{2}$. In view of the PV varieties the generated power additionally varied however it satisfies the load requirements. In the figure 15(b) speaks to the produced irradiance of the PV that is $1500 \mathrm{~W}$. The PV generated power can be utilized to fulfill the load request in the framework side. In this system, load demand can be is expected to the consistent load of $8000 \mathrm{~W}$.

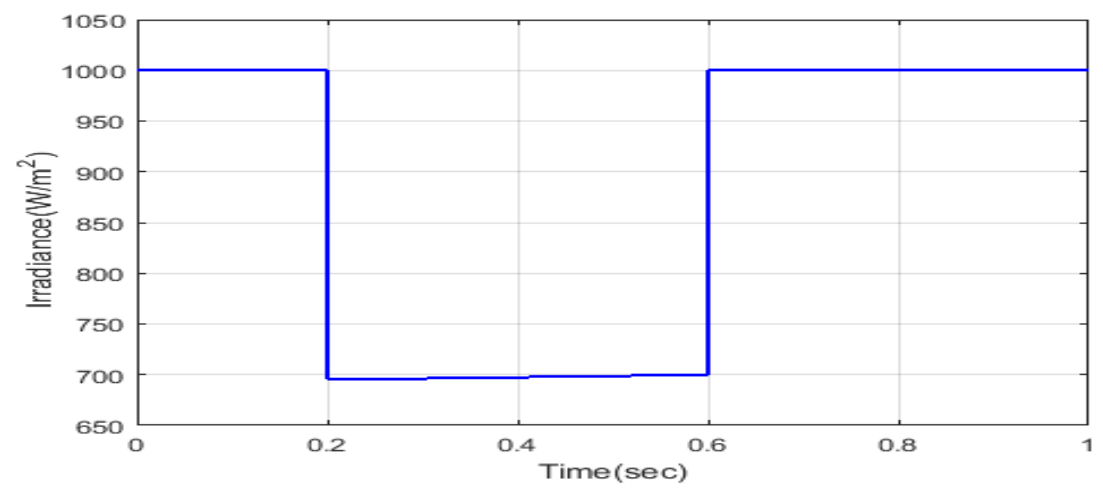

(a) 


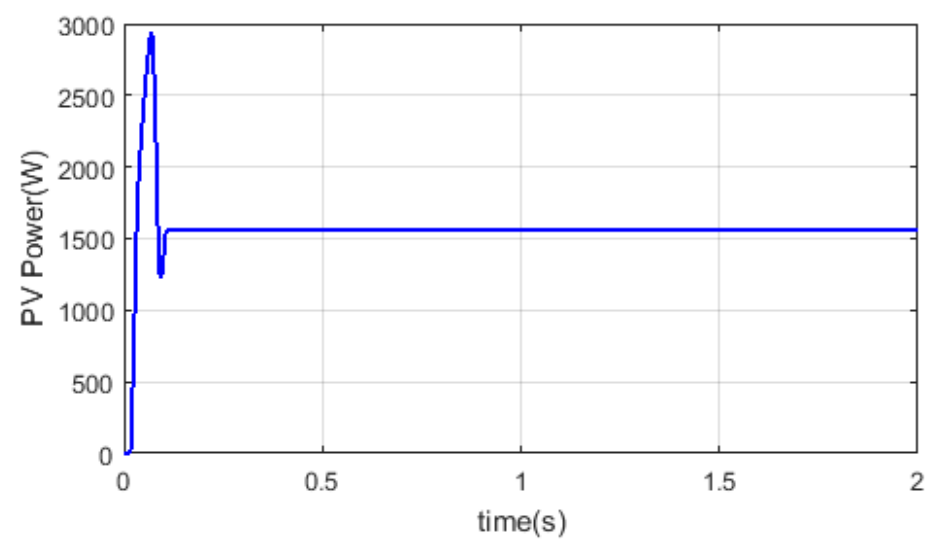

(b)

Figure 15: Solar irradiance and (b) power for PV

In figure 16 speaks to the battery discharge and charge power. The battery storage maximum energy is $4000 \mathrm{~W}$. The capacity cycle is relies on the abundance power structure the generation power which used to compensate the load request. Idea of battery can be characterized as once overabundance power have in the generator side, the battery is operating as the charging mode and when required the ability to fulfill the need in matrix side, the battery can be releasing operation. In view of the method of activity the battery can be used to provide essential generated power. The interest isn't redress, at first the battery is giving the ability to control the power management.

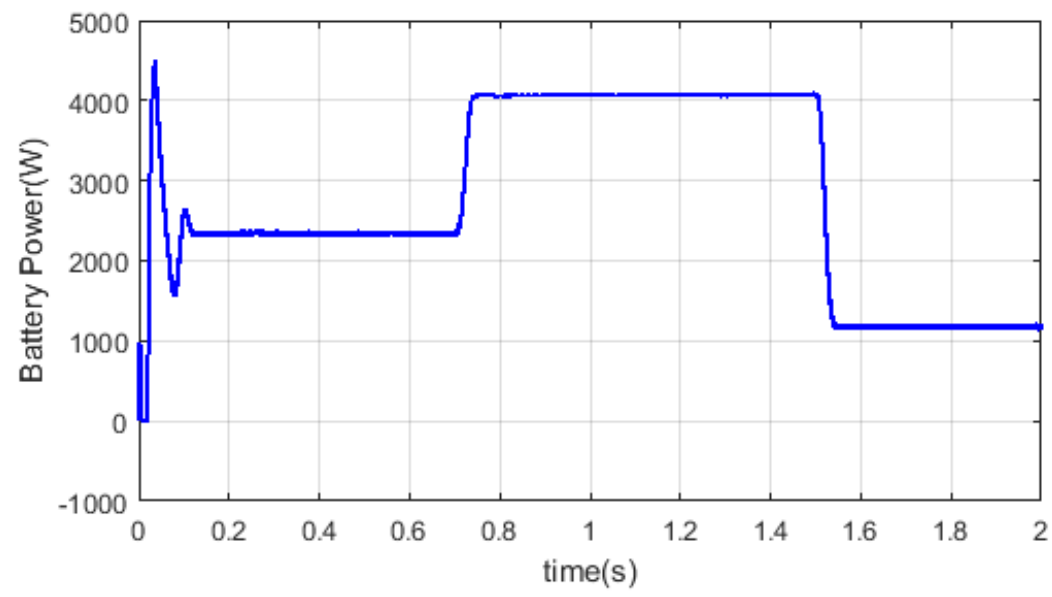

Figure. 16 battery power 


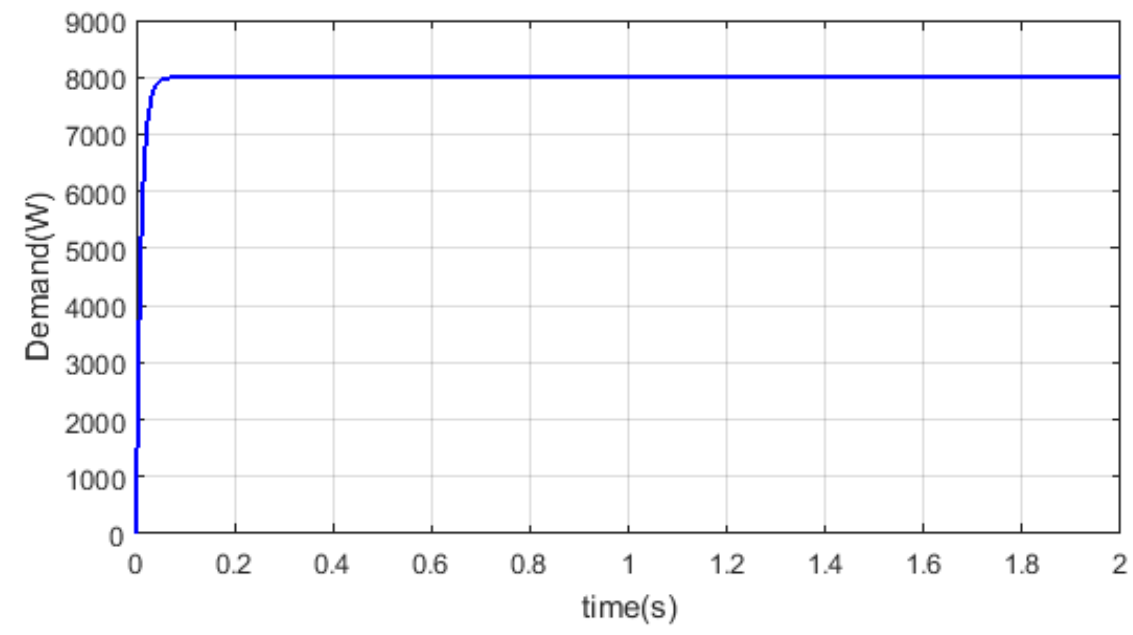

(a)

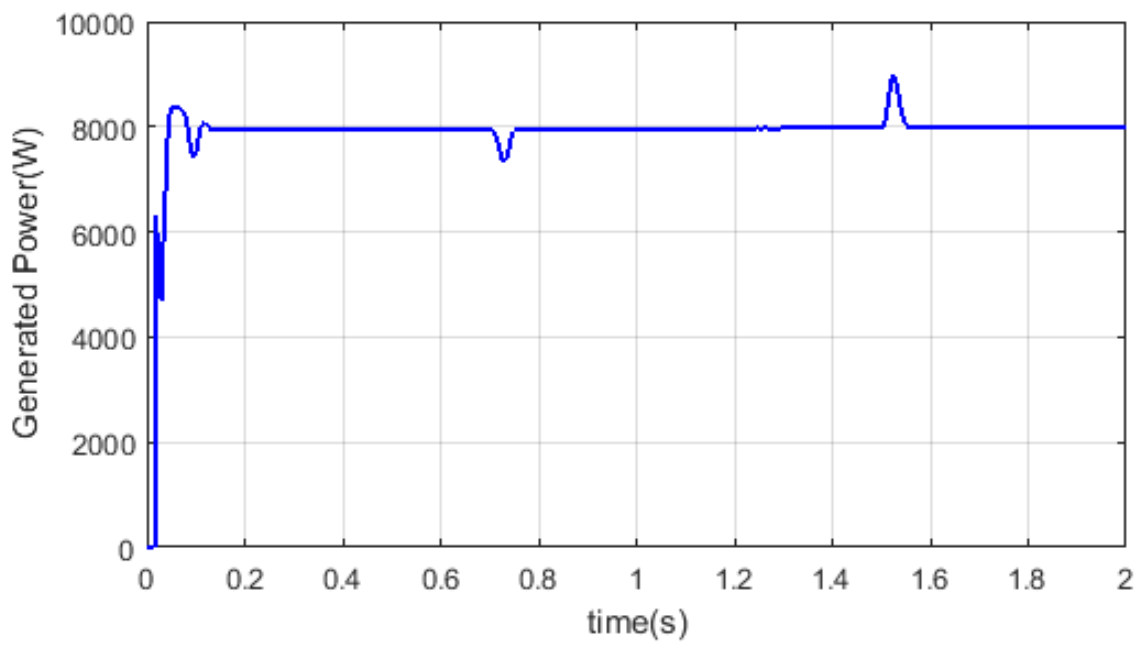

(b)

Figure. 17 (a) load request and (b) generated power 


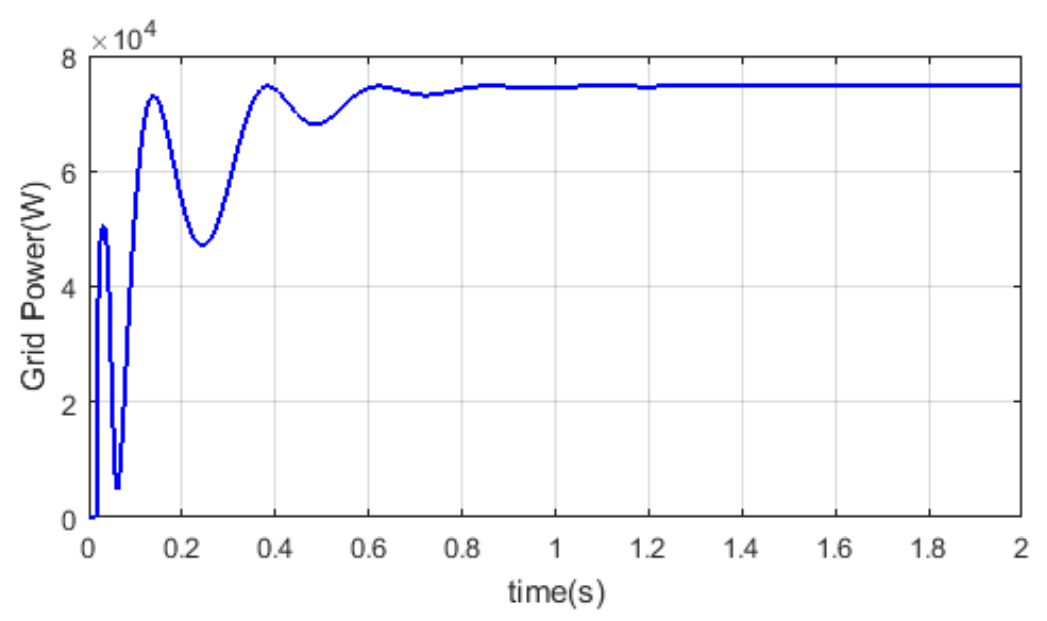

Figure. 18 output power

Subsequently the demand and complete generated power is portrayed in the figure 17. Our principle is provide the load requirements when load most extreme or least. And, optimal power management control examination is accomplishing the remuneration of load demand through reactive and real power control. Load request is repaid by the generated energy of the sustainable sources, battery. This situation, complete generated power can be $8000 \mathrm{~W}$. In reality the irradiance is $8000 \mathrm{~W}$, generated power likewise $8000 \mathrm{~W}$, hence the load request can be redressed. The power management control is accomplished by the usage of the circulated control of reactive and real power with EPO-PSO calculation. The examination of the yield power is portrayed in figure 18. The correlation examination of the generated power and dc link voltage utilizing different techniques can be portrayed under the two cases in the accompanying area 4.2.

\subsection{Comparison Analysis}

This sub section, correlation examination can be accomplished intended for the over two cases. Examination can be finished with the previous methods such as ACO in addition of PSO calculations. Generated power and dc link voltage can be contrasted and the existing strategy. And examination can be utilized to think about the capacity of the projected strategy. 


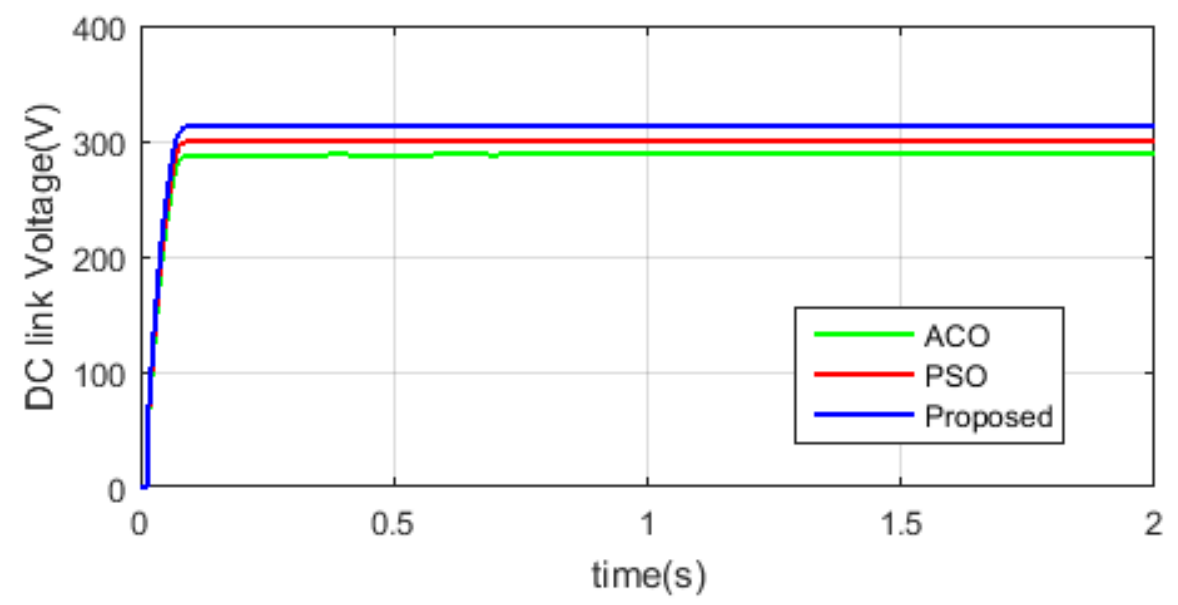

(a)

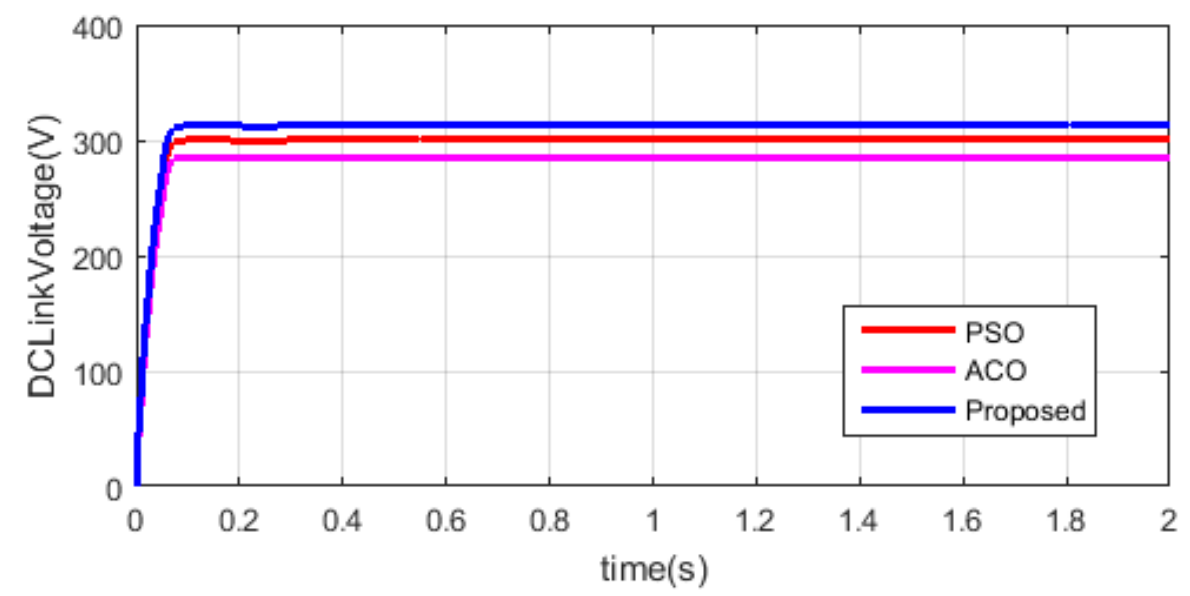

(b)

Figure. 19 (a) case 1-DC link voltage and (b) case 2- DC link voltage

The proposed strategy adequacy is determined dependent on the ascent time esteem. In view of the figure 19(a), the projected method peak time is $t=0.01-2.0 \mathrm{sec}$ at $310 \mathrm{~V}$. PSO technique rise time is $\mathrm{t}=0.015-2.0 \mathrm{sec}$ at $300 \mathrm{~V}$. ACO technique rise time is $\mathrm{t}=0.016-2.0 \mathrm{sec}$ at $280 \mathrm{~V}$ is achieved. Dc link voltage investigation can be contrasted and two previous existing calculations in ACO and PSO. In correlation of the projected calculation have a greater proficiency; it can be demonstrating the pictorial portrayal. In figure 19 (b) the projected procedure rise time is $t=0.01-2.0 \mathrm{sec}$ at $310 \mathrm{~V}$. And, PSO strategy rise time is $\mathrm{t}=0.015-2.0 \mathrm{sec}$ at $300 \mathrm{~V}$. ACO strategy rise time is $\mathrm{t}=0.016-2.0 \mathrm{sec}$ 
at $270 \mathrm{~V}$ is occurred. The dc connect voltage is $310 \mathrm{~V}$, the presented technique just meet the degree of voltage and keep up at steady yet the current strategies are not meet the consistent estimation of the dc link voltage. In view of the voltage level, the proposed technique is having high effectiveness with looking at existing strategies.

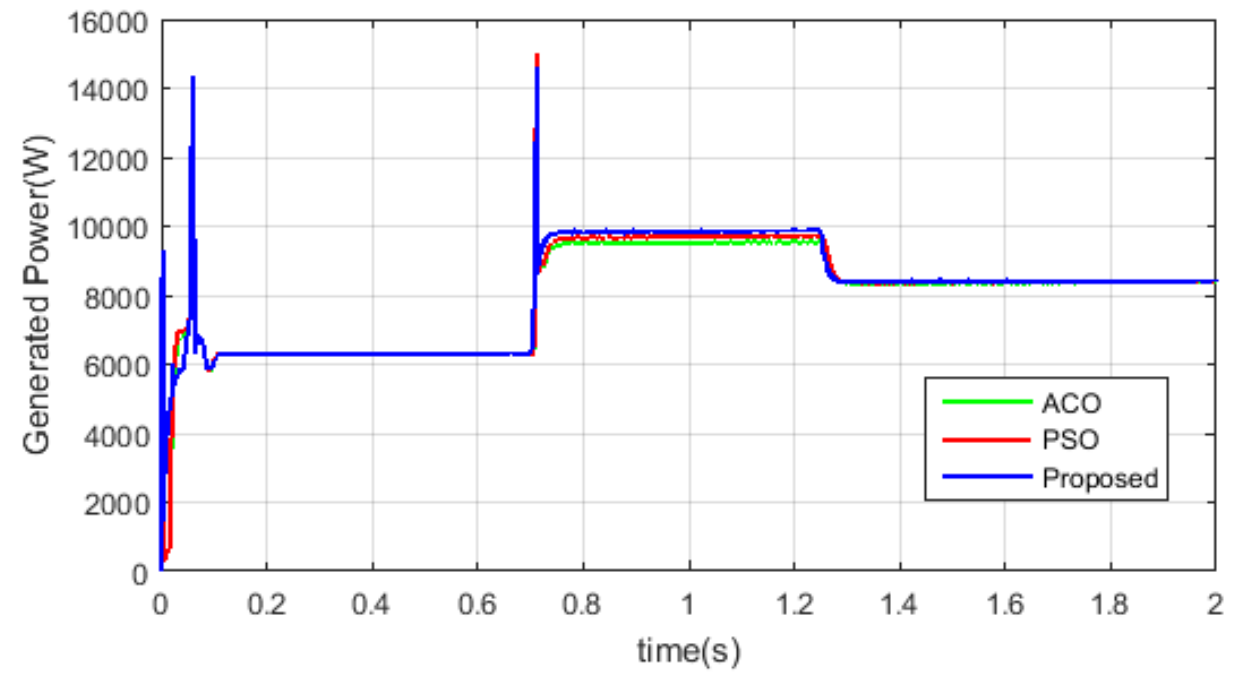

(a)

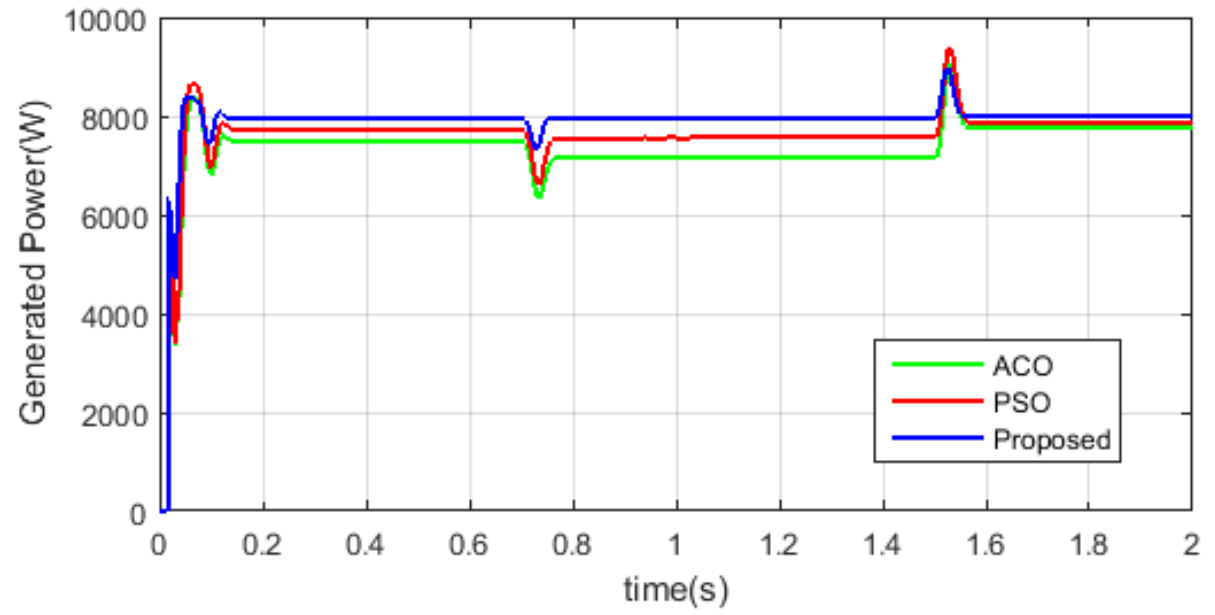

(b)

Figure. 20 (a) case 1 comparison analysis and (b) case 2 comparison analysis 
Figure 20 represents the generated power comparison analysis of case 1 and case 2 . The proposed method should compensate the load demand in the grid side which validated by comparison analysis. The proposed method capability is validated by compared with existing methods such as ACO as well as PSO methods.

The figure $20(\mathrm{~b})$, the demand is $8000 \mathrm{~W}$ but the ACO method only compensate load demand of 6800W. Similarly, the PSO method is compensated the load demand of $7200 \mathrm{~W}$ respectively. From the analysis, the proposed method only compensate the complete load demand of system. Hence, we can concluded, the proposed method is achieves better efficiency to attain the power management. In the proposed technique based distributed control for power management assessed generated power that has been exploited as response indication in load demand. From these comparison analysis, the proposed method is completely achieves the best result to compensate load demand. In the results figures, the proposed and existing methods are clearly described with different colors.

Figure 20 speaks to the generated power correlation examination of case 1 and case 2 . The examination investigation is determined dependent on the load demand. To demonstrate the viability of the proposed strategy, this is contrasted and the existing strategies, for example, PSO and ACO technique individually. The figure 20(b), the load demand is $8000 \mathrm{~W}$ however the ACO technique just compensated the interest up to $6800 \mathrm{~W}$ and the PSO additionally fulfilled the 7200 W request as it were. In view of the interest the proposed strategy fulfills the full interest after the network side. Hence, the presented method has a greater productivity contrasting with the previous designed strategies. In the proposed procedure based appropriated control for power the executives evaluated generated power that has been misused as reaction sign in load demand. The correlation examination of considering for presented the aptitude of the projected method dependent on the worth. The examinations investigation plainly portrayed in the figures and strategies at different shadings.

\section{Conclusion}

In the paper, PV with SEPIC and ZSI is planned in the MATLAB/Simulink stage. These are associated among framework and load demand. The conveyed control technique is planned 
dependent on the FOPID regulator with help of hybrid approach for producing the optimal signals for ZSI and SEPIC. Here the exhibition of the ZSI and SEPIC is discovered to be good for two distinctive control plans for power the executives and most extreme force following. The proposed method is computed to be achieved with their two control methods such as power management in addition maximum power tracking. Additionally, active as well as reactive power compensation based on environmental conditions of PV system. In this scenario, the proposed method is working efficient by delivering real and reactive power in the system. Case 1 deliberate consistent irradiance condition. This was never really power the executives in the output voltage of inverter. Outcomes demonstrate great execution of the control techniques in two cases such as consistent and changed cases. And, regulator can be appeared towards extricate most extreme force on or after the PV modules through keeping up the DC-link voltage at the reference value at the two cases. The exhibitions of regulators, during change conditions in info capacity to the inverters, because of variations in the accessibility of sun based irradiance, can be discovered towards acceptable in environmental conditions. The proposed strategy is contrasted and the current strategies, for example, base regulator, ACO and PSO procedures. By using the proposed regulator, the dc link voltage is exceptionally directed when contrasted and different strategies. The load demand and generated powers are investigated utilizing the proposed and existing techniques. From the general examination, the proposed strategy accomplishes better outcomes when contrasted with different methods.

\section{Ethics declarations}

\section{Conflict of interest}

The author declares that he has no conflict of interest.

\section{Ethical approval}

This article does not contain any studies with human participants or animals performed by the author.

\section{Informed consent:}

Informed consent was obtained from all individual participants included in the study. 


\section{Author information:}

Research scholar, Department of EEE, Anna University, Chennai, TamilNadu, India. Mrs. Vidhya. M

Professor, Department of EEE, Bannari Amman Institute of Technology, Sathyamanglam, TamilNadu, India.

\section{Dr. Senthil Kumar. R}

Corresponding author: Mrs. Vidhya. M

\section{References}

[1].Castilla, M., Carlos Bordons, and Antonio Visioli, "Event-based state-space model predictive control of a renewable hydrogen-based microgrid for office power demand profiles", An International Journal of Power Sources, Vol.450, 2020.

[2]. Varshney, Gunjan, D. S. Chauhan, and M. P. Dave, "Grid connected solar PV system using carbon material based DYE sensitized solar cells", In proceedings of Materials Today, 2020

[3].Bashir, SafiaBabikir, Hasan A.Zidan, and Zulfiqar Ali Memon, "Power balancing of grid connected PV system based on MMC under different irradiation conditions", An International Journal of Electrical Power and Energy Systems, Vol.117, 2020.

[4].Ismail, Mohamed M., and Ahmed F. Bendary, "Smart battery controller using ANFIS for three phase grid connected PV array system", An International Journal of Mathematics and Computers in Simulation, Vol.167, pp.104-118, 2020.

[5].Saha, Sajeeb, Md EnamulHaque, C. P. Tan, Md Apel Mahmud, Mohammad TaufiqulArif, S. Lyden, and N. Mendis, "Diagnosis and mitigation of voltage and current sensors malfunctioning in a grid connected PV system", An International Journal of Electrical Power \& Energy Systems, Vol.115, 2020

[6].Singh, Shakti, Prachi Chauhan, and NirbhowJap Singh, "Capacity optimization of grid connected solar/fuel cell energy system using hybrid ABC-PSO algorithm", An International Journal of Hydrogen Energy, 2020

[7]. Winston, D. Prince, S. Kumaravel, B. Praveen Kumar, and S. Devakirubakaran. "Performance improvement of solar PV array topologies during various partial shading conditions”, An International Journal of Solar Energy, Vol.196, pp.228-242, 2020 
[8].Verma, Pallavi, Rachana Garg, and Priya Mahajan, "Asymmetrical interval type-2 fuzzy logic control based MPPT tuning for PV system under partial shading condition”, An International journal of ISA Transactions, 2020.

[9].VS, B.R. and Devadhas, G.G., "Design and development of new control technique for standalone PV system", An International Journal of Microprocessors and Microsystems, Vol.72, 2020.

[10]. Najafi-Shad, Sajad, S. MasoudBarakati, and AmirnaserYazdani, "An effective hybrid wind-photovoltaic system including battery energy storage with reducing control loops and omitting PV converter", An International Journal of Energy Storage, Vol.27, 2020

[11]. Zhiping Cheng, Zhongwen Li, Jing Liang, Jikai Si, Lianghui Dong and Jinfeng Gao, "Distributed coordination control strategy for multiple residential solar PV systems in distribution networks", An International Journal of Electrical power and energy systems, Vol.117, 2020

[12]. Şahin, Mustafa Ergin, "A photovoltaic powered electrolysis converter system with maximum power point tracking control", An International Journal of Hydrogen Energy, 2020

[13]. Jain, A. Arther, B. Justus Rabi, and S. S. Darly, "Application of QOCGWO-RFA for maximum power point tracking (MPPT) and power flow management of solar PV generation system", An International Journal of Hydrogen Energy, 2020

[14]. Eltamaly, Ali M., M. S. Al-Saud, and A. G. Abo-Khalil, "Performance Improvement of PV Systems' Maximum Power Point Tracker Based on a Scanning PSO Particle Strategy", An International Journal of Sustainability, Vol.12, No.3, 2020

[15]. Sellali, M., A. Betka, and A. Djerdir, "Power management improvement of hybrid energy storage system based on $\mathrm{H} \infty$ control", An International Journal of Mathematics and Computers in Simulation, Vol.167, pp.478-494, 2020

[16]. Hossain, Md Alamgir, Hemanshu Roy Pota, Stefano Squartini, and Ahmed FathiAbdou, "Modified PSO algorithm for real-time energy management in grid-connected microgrids", An International journal of Renewable energy, Vol.136, pp.746-757, 2019.

[17]. Roy, Kallol, Kamal Krishna Mandal, and Atis Chandra Mandal, "Ant-Lion Optimizer algorithm and recurrent neural network for energy management of micro grid connected system" An International journal of Energy, Vol.167, pp.402-416, 2019. 
[18]. Abedini, Mohammad, Mohammad H. Moradi, and S. Mahdi Hosseinian, "Optimal management of microgrids including renewable energy scources using GPSO-GM algorithm", Renewable Energy, Vol.90, pp.430-439, 2016

[19]. Tidjani, FadoulSouleyman, AbdelhamidHamadi, Ambrish Chandra, Pragasen Pillay, and AugusteNdtoungou, "Optimization of standalone microgrid considering active damping technique and smart power management using fuzzy logic supervisor", IEEE Transactions on Smart Grid, Vol.8, No.1, pp.475-484, 2017

[20]. Zacharia, L., L. Tziovani, M. Savva, L. Hadjidemetriou, E. Kyriakides, A. D. Bintoudi, A. Tsolakis, "Optimal Energy Management and Scheduling of a Microgrid in GridConnected and Islanded Modes", In proceedings of International Conference on Smart Energy Systems and Technologies (SEST), pp.1-6, 2019.

[21]. Gholinejad, Hamid Reza, AbdolahLoni, JafarAdabi, and MousaMarzband. "A hierarchical energy management system for multiple home energy hubs in neighborhood grids", An International Journal of Building Engineering, Vol.28, 2020

[22]. Matayoshi, Hidehito, Mitsunaga Kinjo, Shriram S. Rangarajan, Girish GanesanRamanathan, Ashraf M. Hemeida, and TomonobuSenjyu, "Islanding operation scheme for DC microgrid utilizing pseudo Droop control of photovoltaic system", An International Journal of Energy for Sustainable Development, Vol.55, pp.95-104, 2020

[23]. Elkazaz, Mahmoud, Mark Sumner, and David Thomas, "Energy management system for hybrid PV-wind-battery microgrid using convex programming, model predictive and rolling horizon predictive control with experimental validation", An International Journal of Electrical Power and Energy Systems, Vol.115, 2020

[24]. Mr.K.Natarajan, M.Supriya, Y.Abinaya, P.Nandhakumar and G.R.Samrajesh, "Design of a SEPIC converter solar PV system", 2017

[25]. Niapour, SA KH Mozaffari, S. Danyali, M. B. B. Sharifian, and M. R. Feyzi, "Brushless DC motor drives supplied by PV power system based on Z-source inverter and FL-IC MPPT controller", An International Journal of Energy Conversion and Management, Vol.52, No.89, pp.3043-3059, 2011.

[26]. Ellabban, Omar, and Haitham Abu-Rub, "An overview for the Z-Source Converter in motor drive applications", An International Journal of Renewable and Sustainable Energy Reviews, Vol.61, pp.537-555, 2016. 
[27]. YaserToghani Holari1, Seyed Abbas Taher and Majid Mehrasa, "Distributed energy storage system-based nonlinear control strategy for hybrid microgrid power management includedwind/PV units in grid-connected operation", An International Journal of transaction an electrical energy systems, 2019

[28]. Anbarasu, Elango, and Adam Raja Basha. "An Improved Power Conditioning System for Grid Integration of Solar Power Using ANFIS based FOPID Controller." An International Journal of Microprocessors and Microsystems, 2020

[29]. Baliarsingh, Santos Kumar, Weiping Ding, Swati Vipsita, and SambitBakshi, "A memetic algorithm using emperor penguin and social engineering optimization for medical data classification", An International Journal of Applied Soft Computing, Vol.85, 2019.

[30]. Li, Jiuhui, Wenxi Lu, Han Wang, Yue Fan, and Zhenbo Chang. "Groundwater Contamination Source Identification Based on a Hybrid Particle Swarm OptimizationExtreme Learning Machine", An International Journal of Hydrology, 2020 
Figures

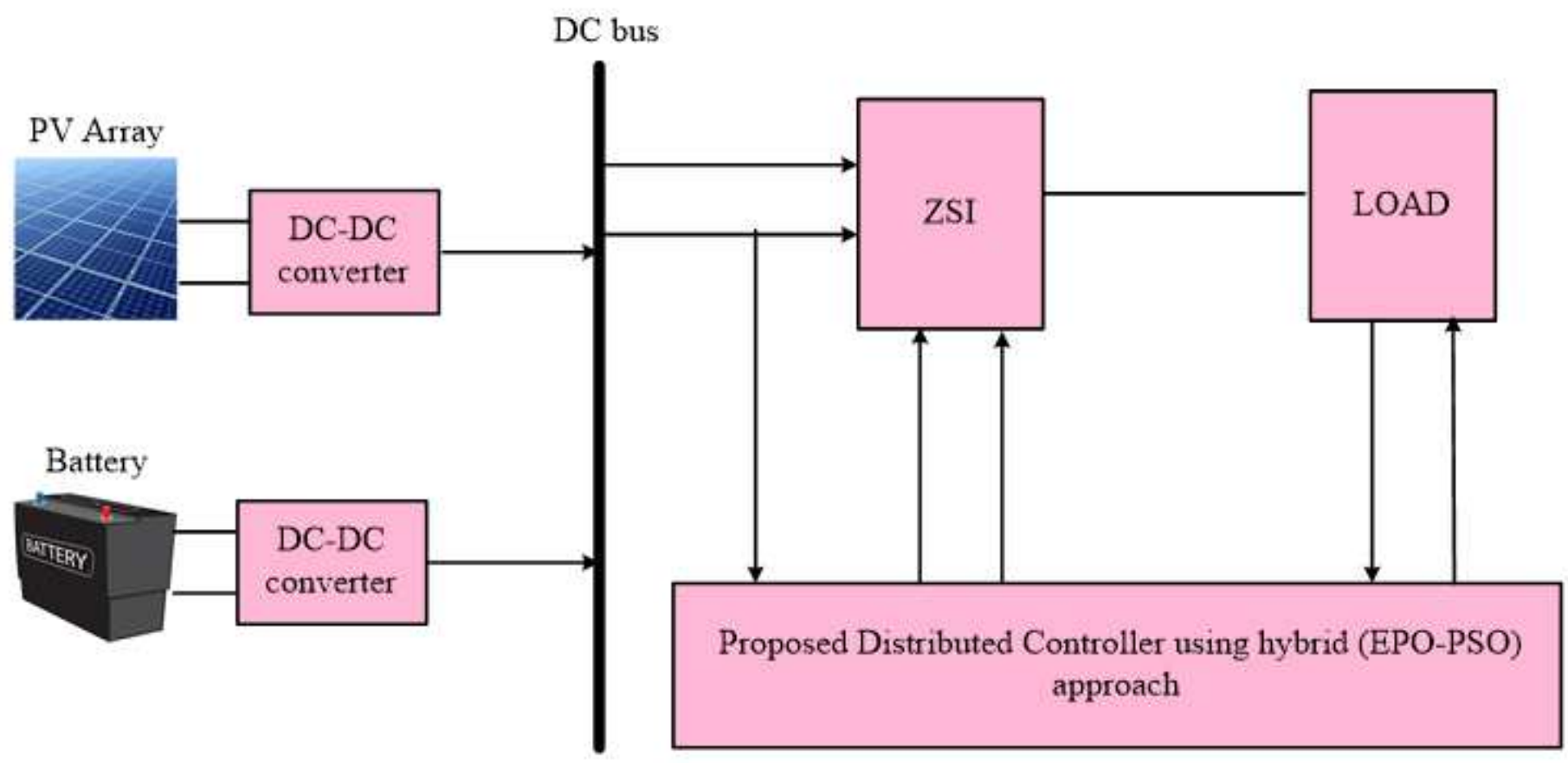

Figure 1

proposed method system model

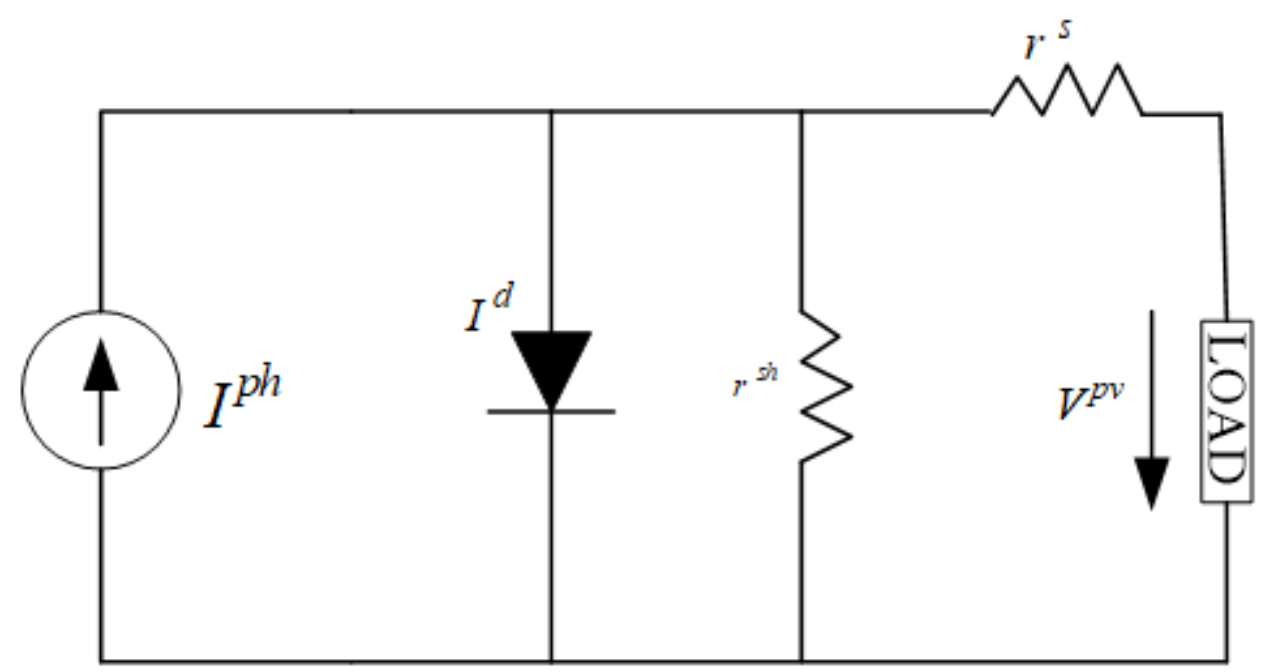

Figure 2

I-V characteristics of the PV system with equivalent circuit model 


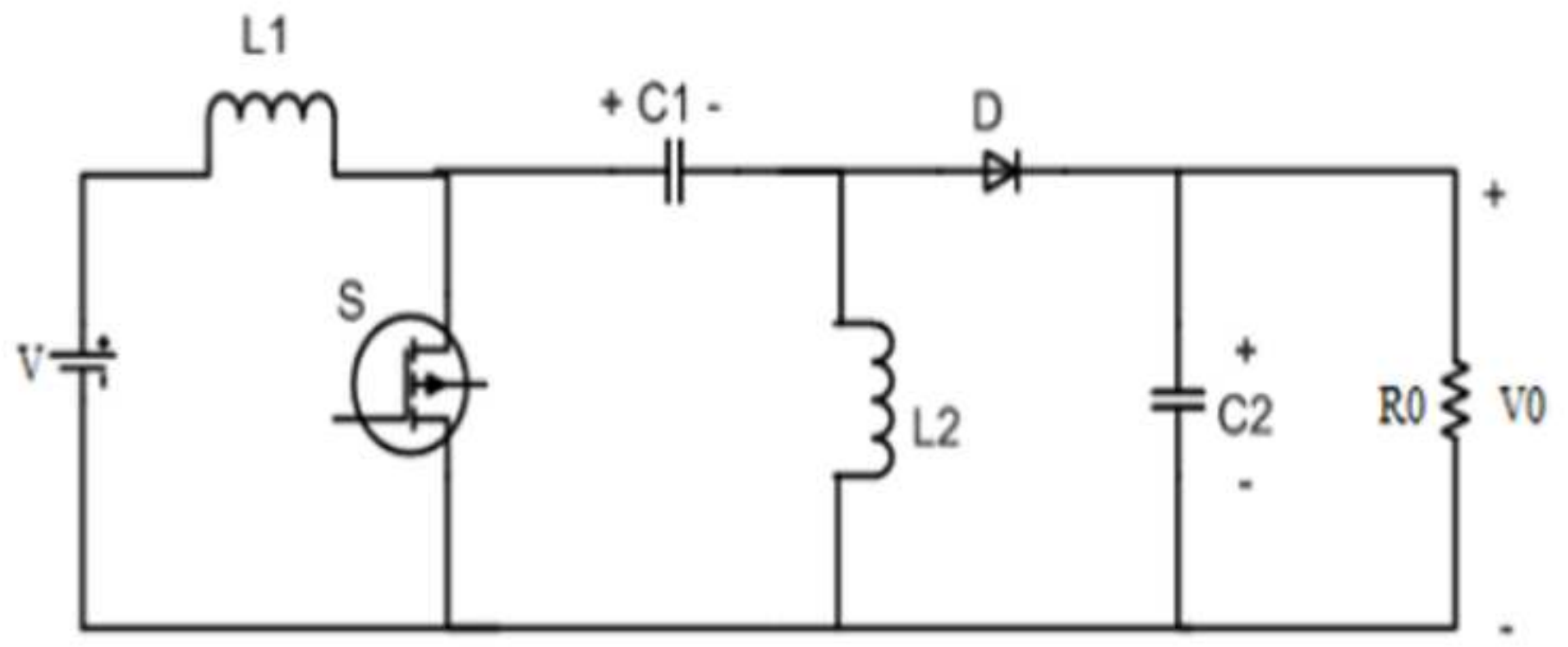

Figure 3

Design of SEPIC converter

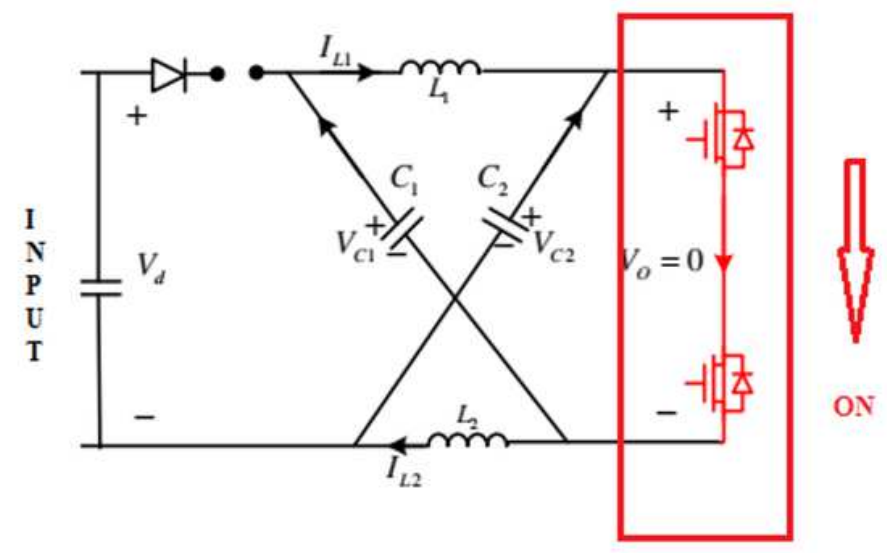

(a)

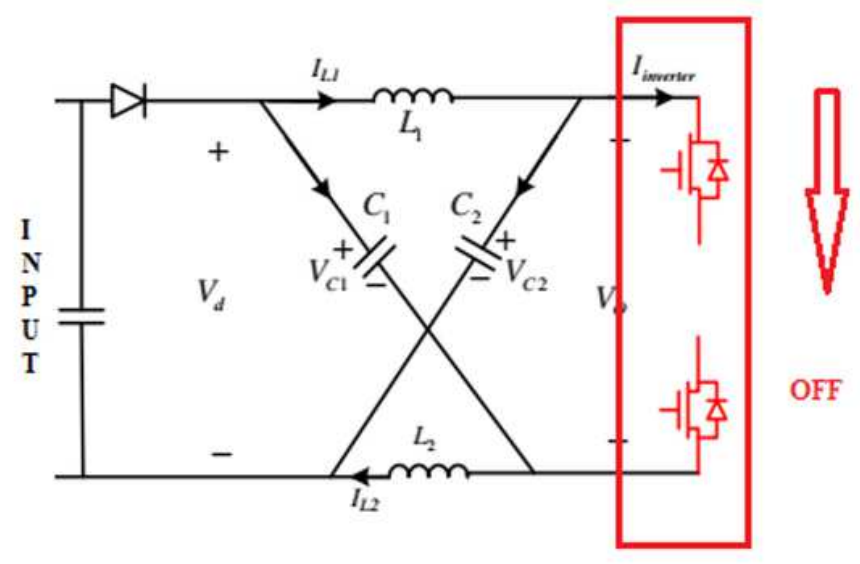

(b)

Figure 4

ZSI equivalent circuit diagram of (a) State of Shoot through and non-shoot through 


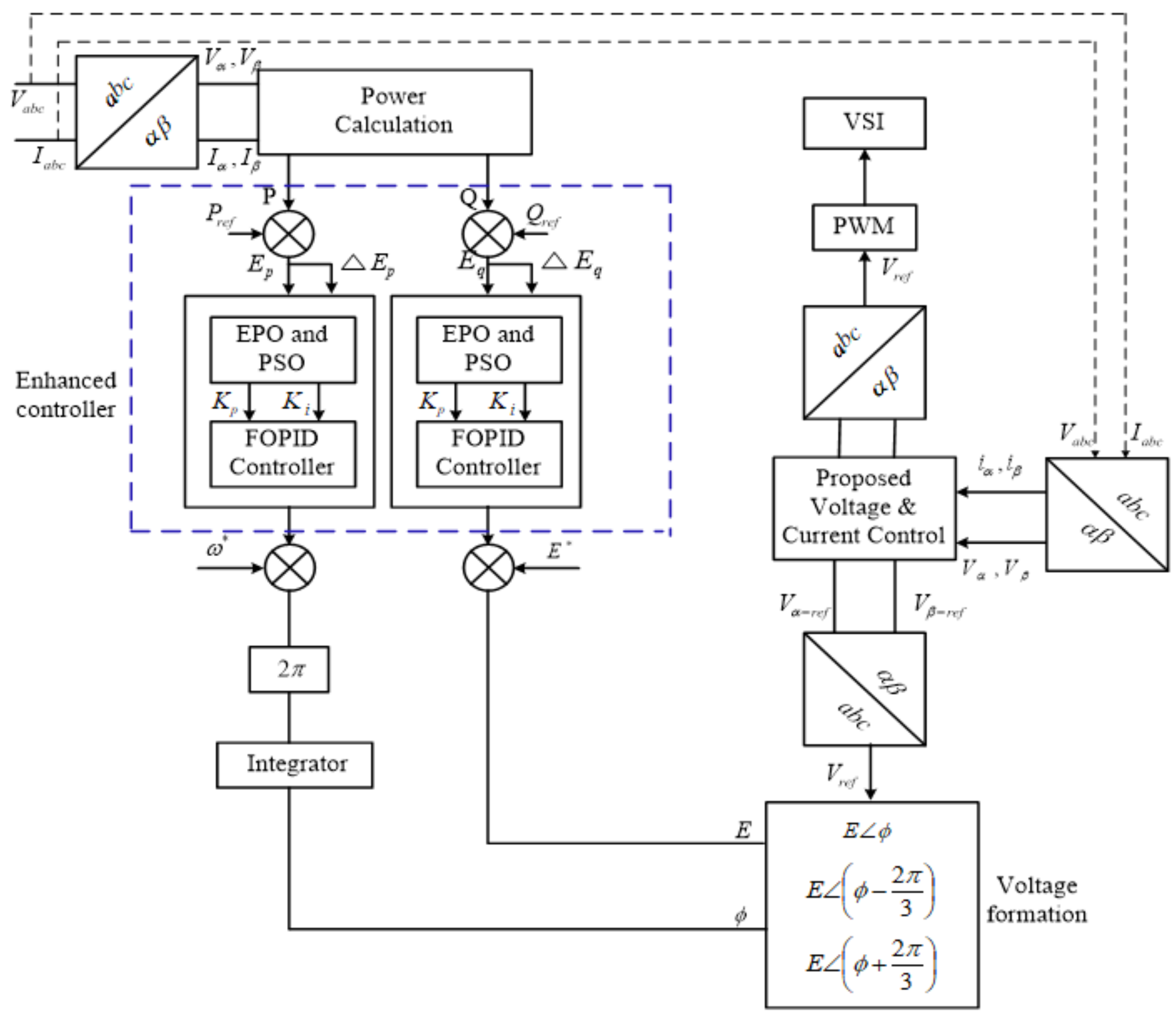

Figure 5

Control schemes of proposed method with FOPID controller 


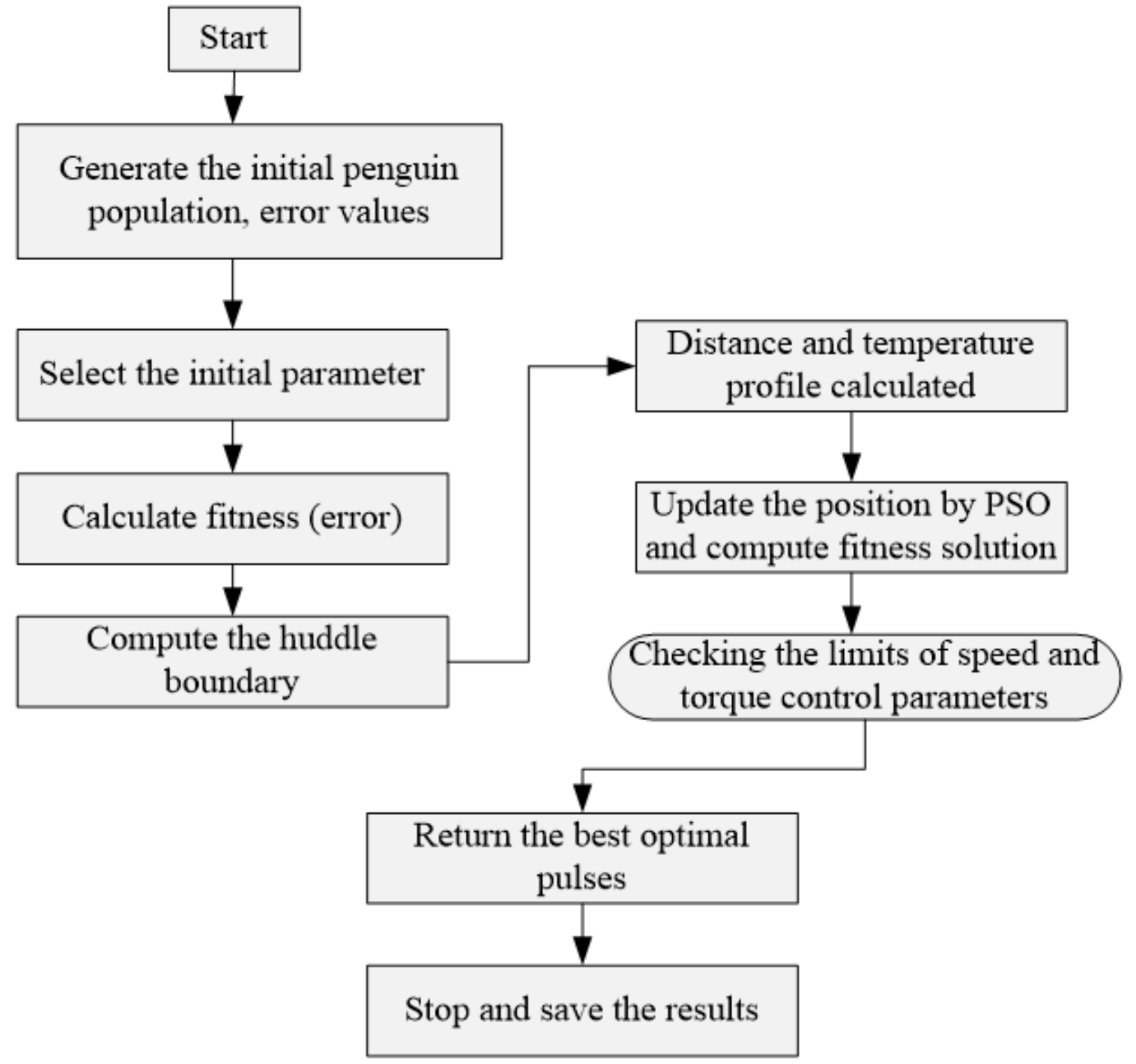

Figure 6

EPO algorithm flowchart 

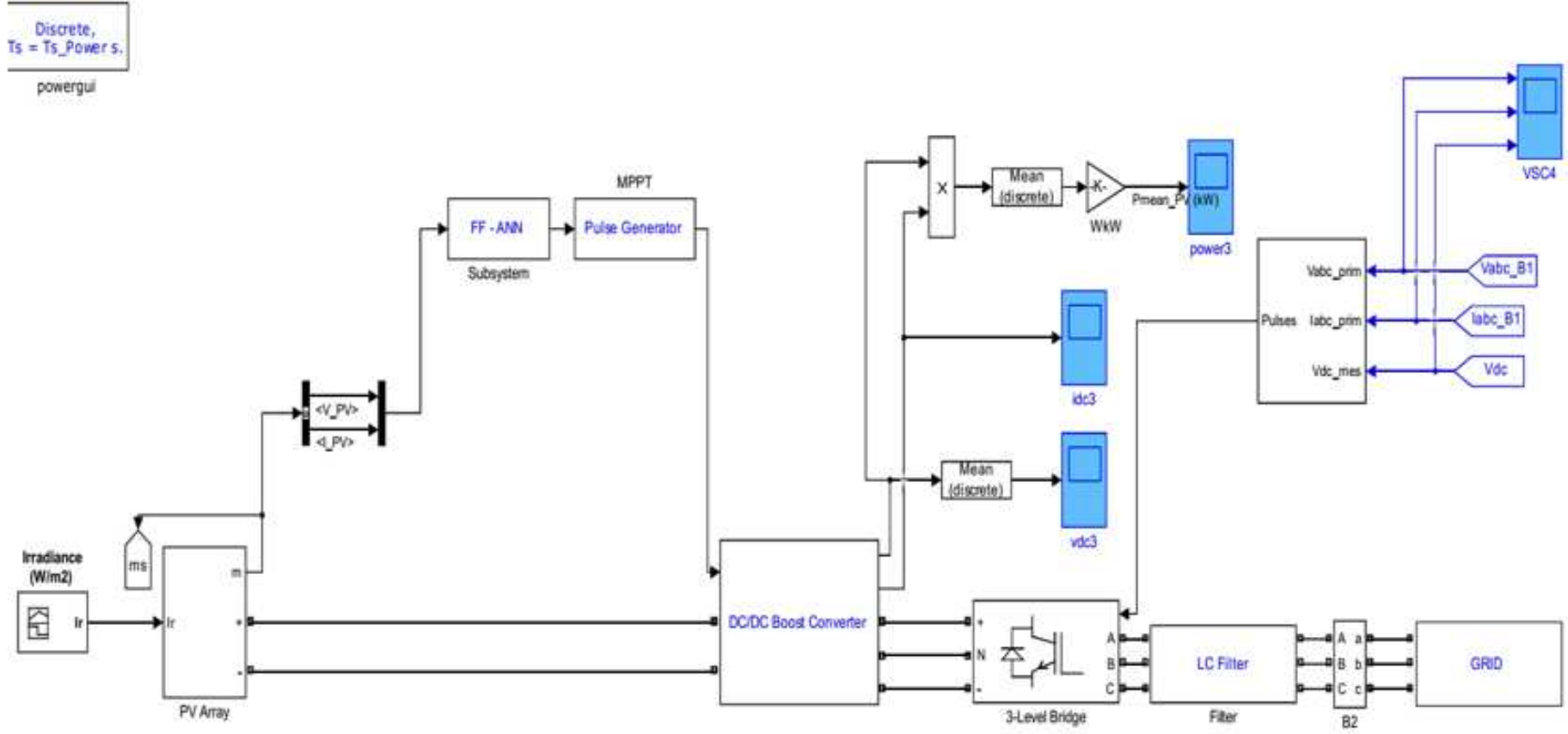

Figure 7

Proposed control system in MATLAB/Simulink

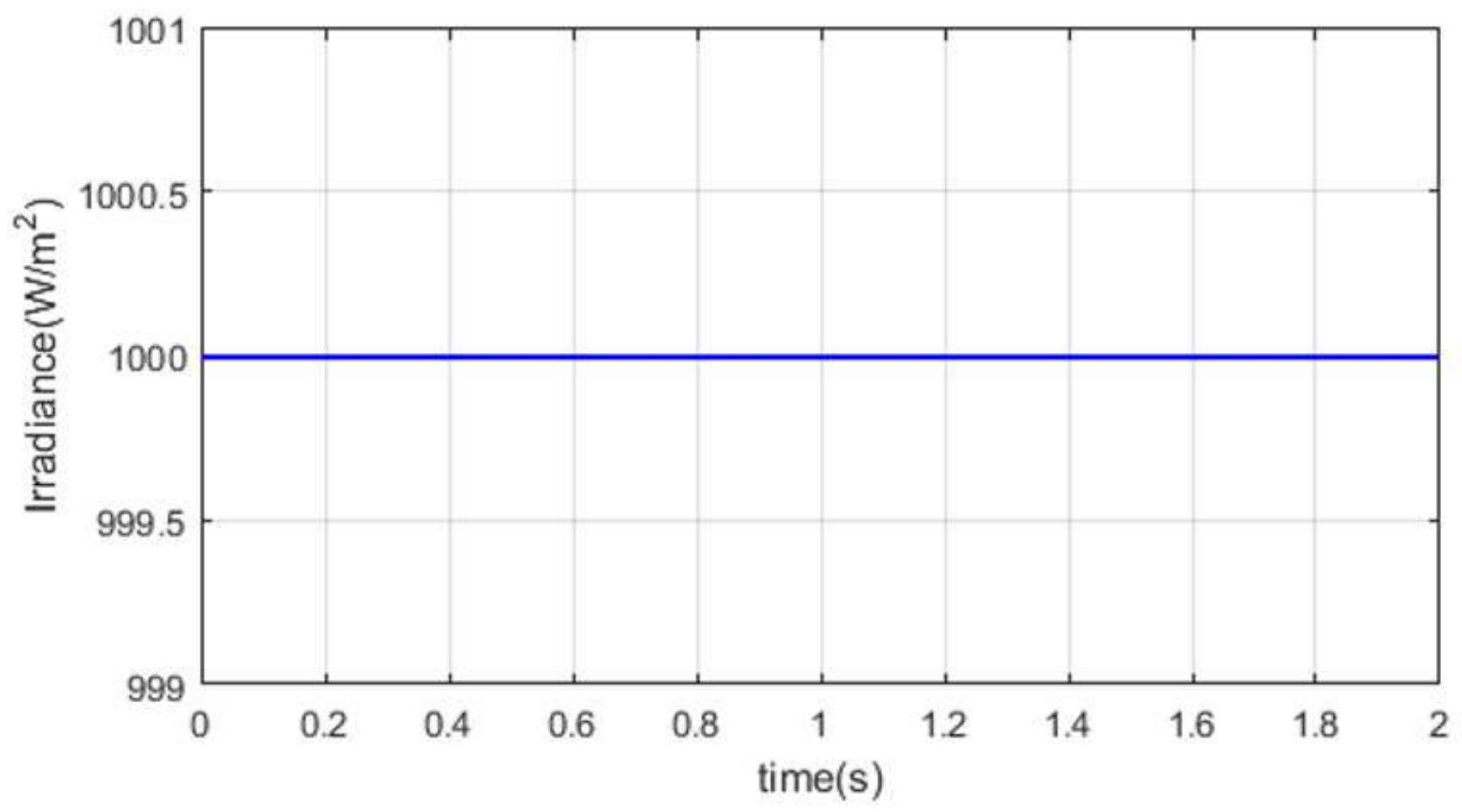

Figure 8

Analysis of Irradiance level 


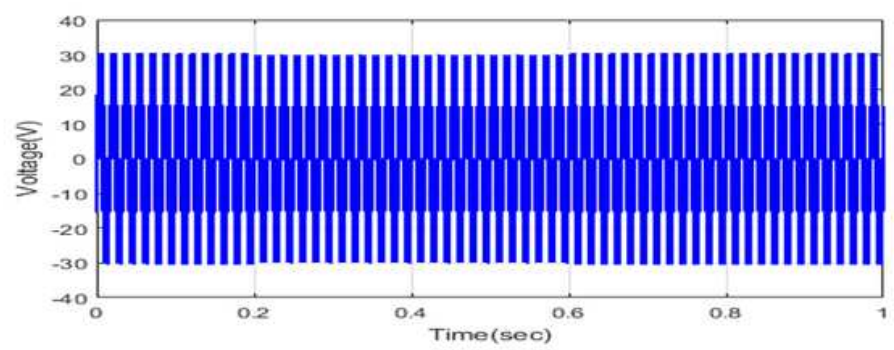

(a)

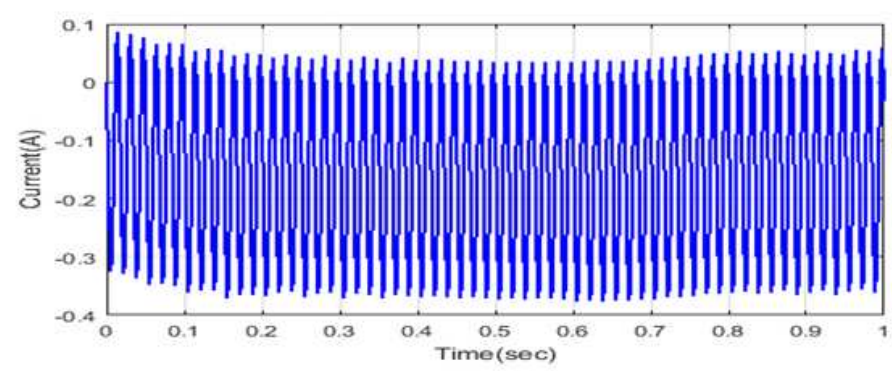

(b)

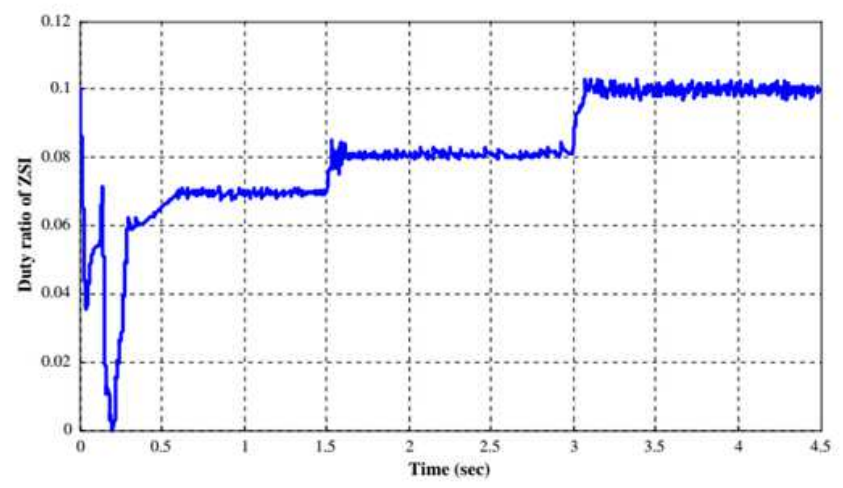

(c)

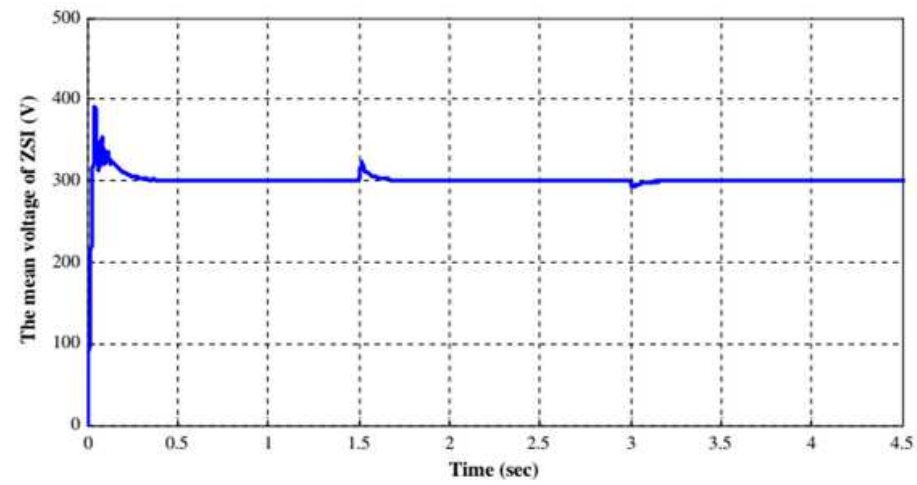

(d)

\section{Figure 9}

Analysis of (a) output voltage of SEPIC converter, (b) current of SEPIC converter,(c) Mean voltage of ZSI and (d) Duty ratio of ZSI using proposed method 


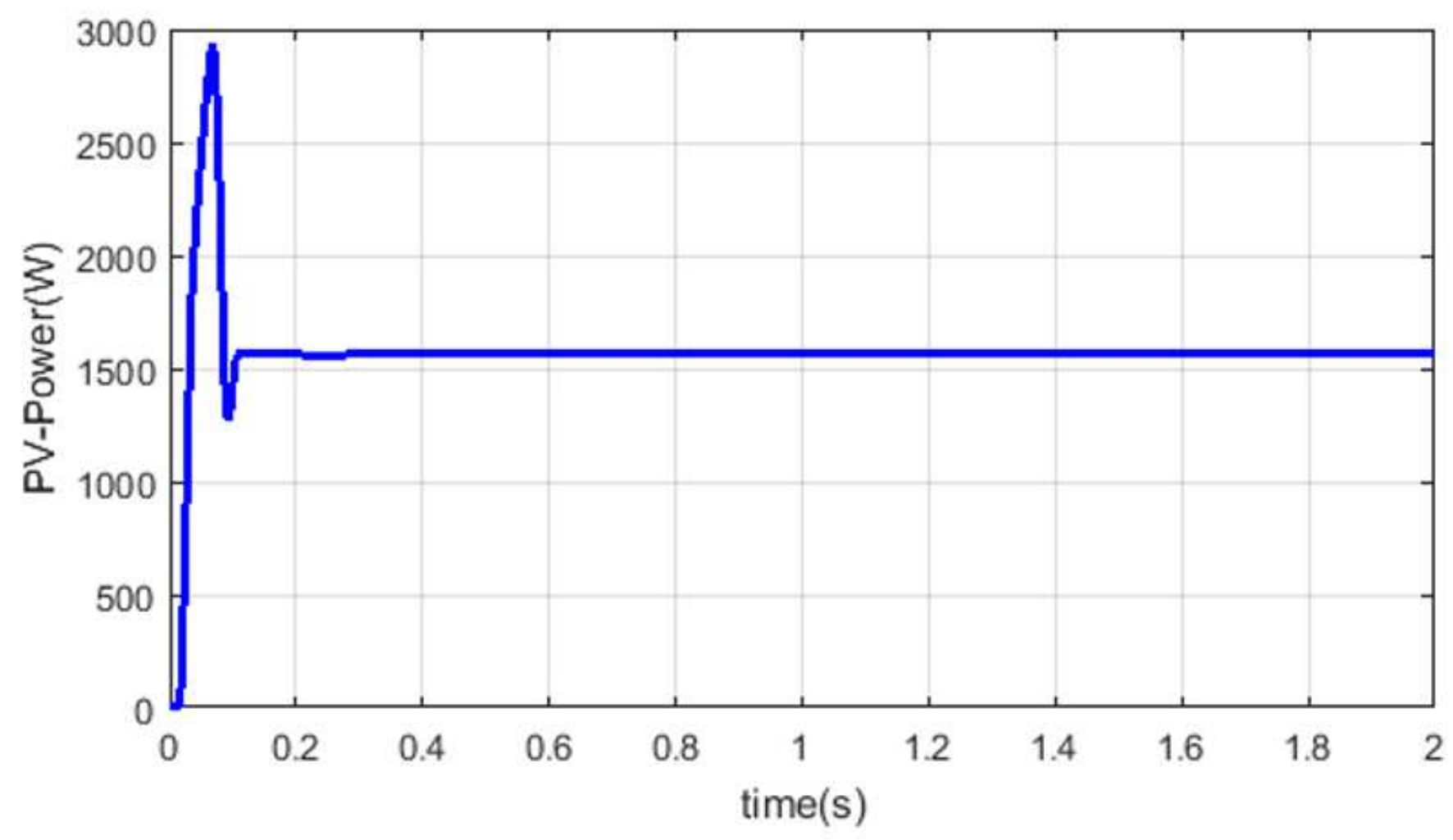

Figure 10

Analysis of PV generated power

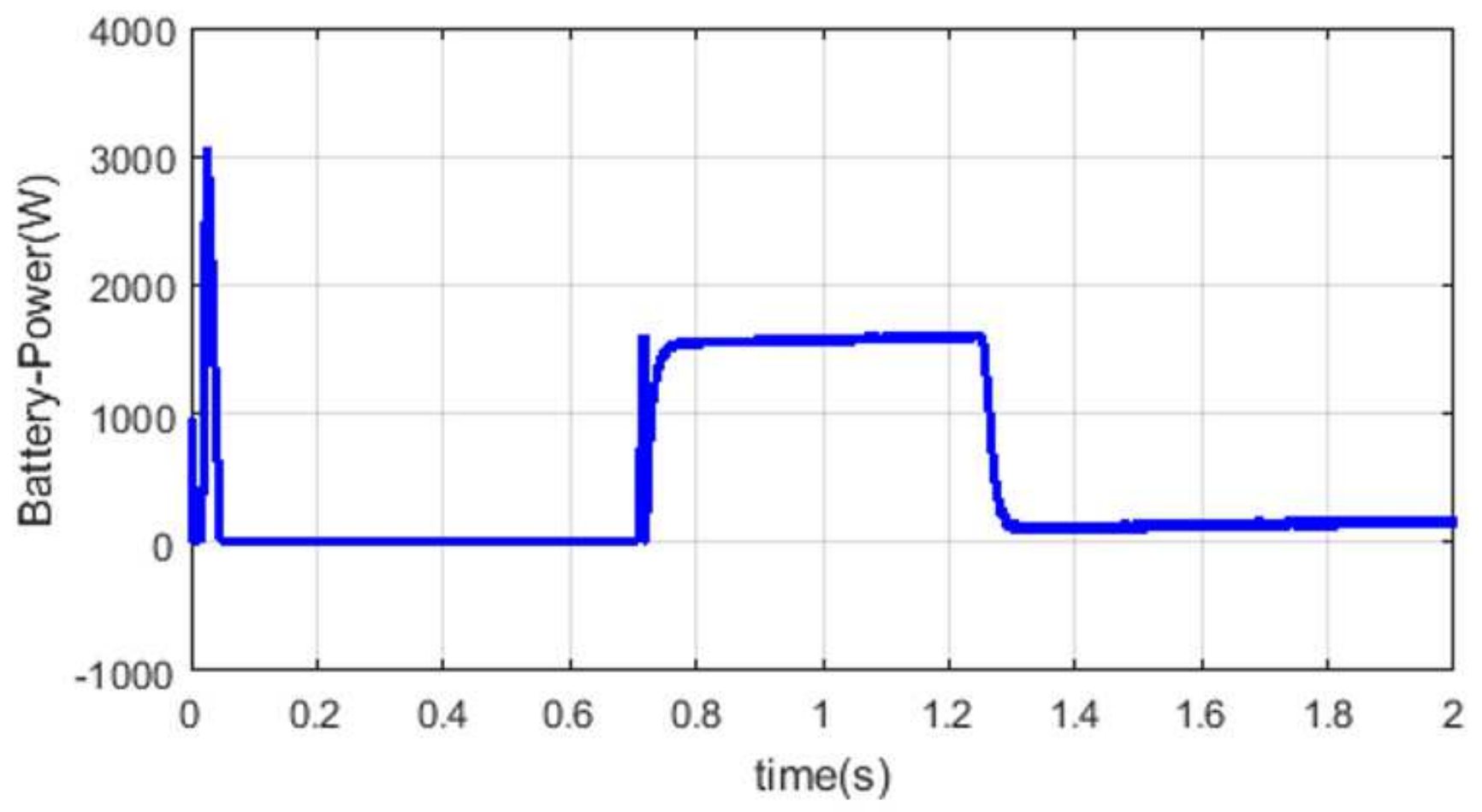

Figure 11 
Analysis of battery charging power

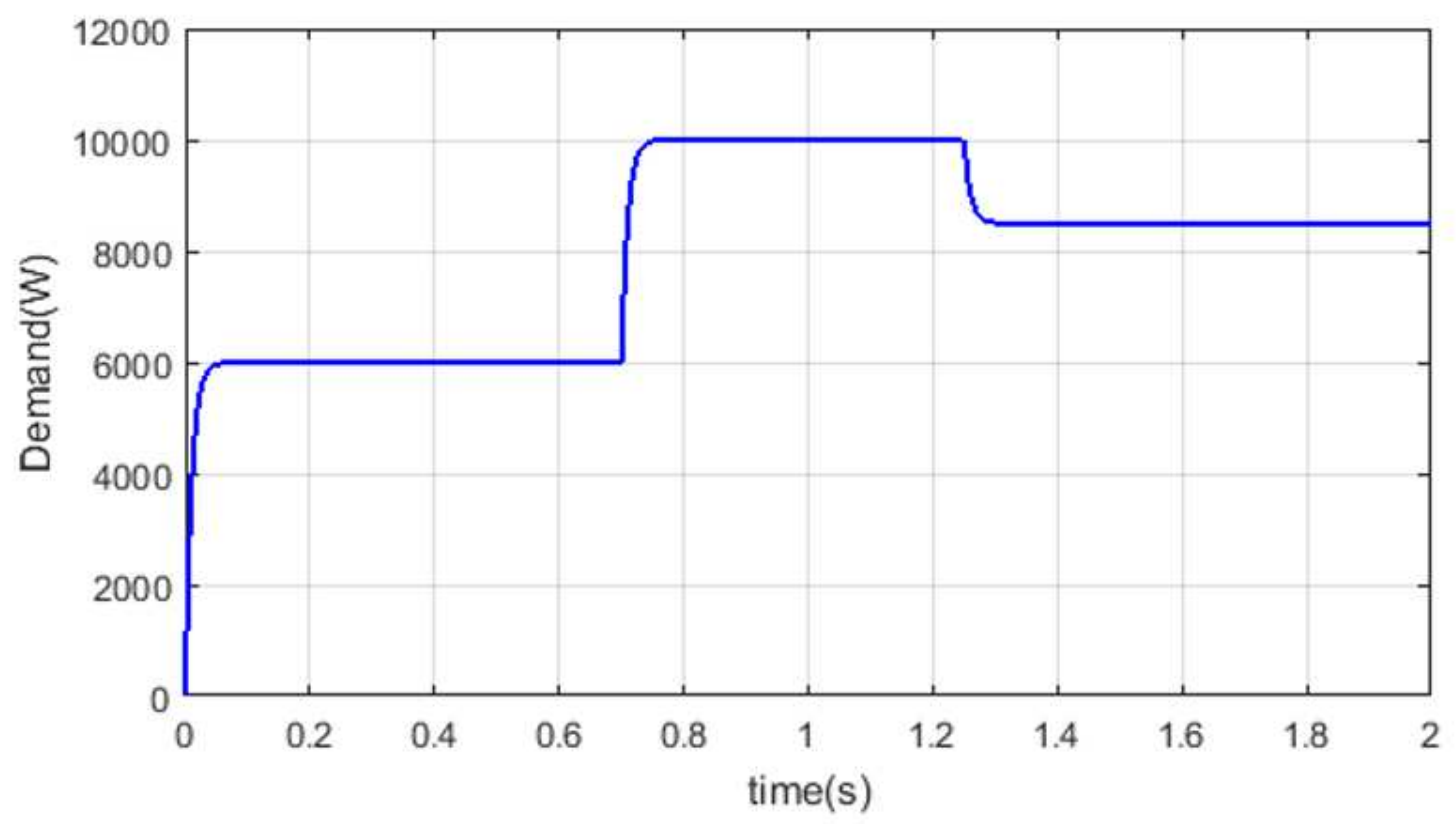

(a)

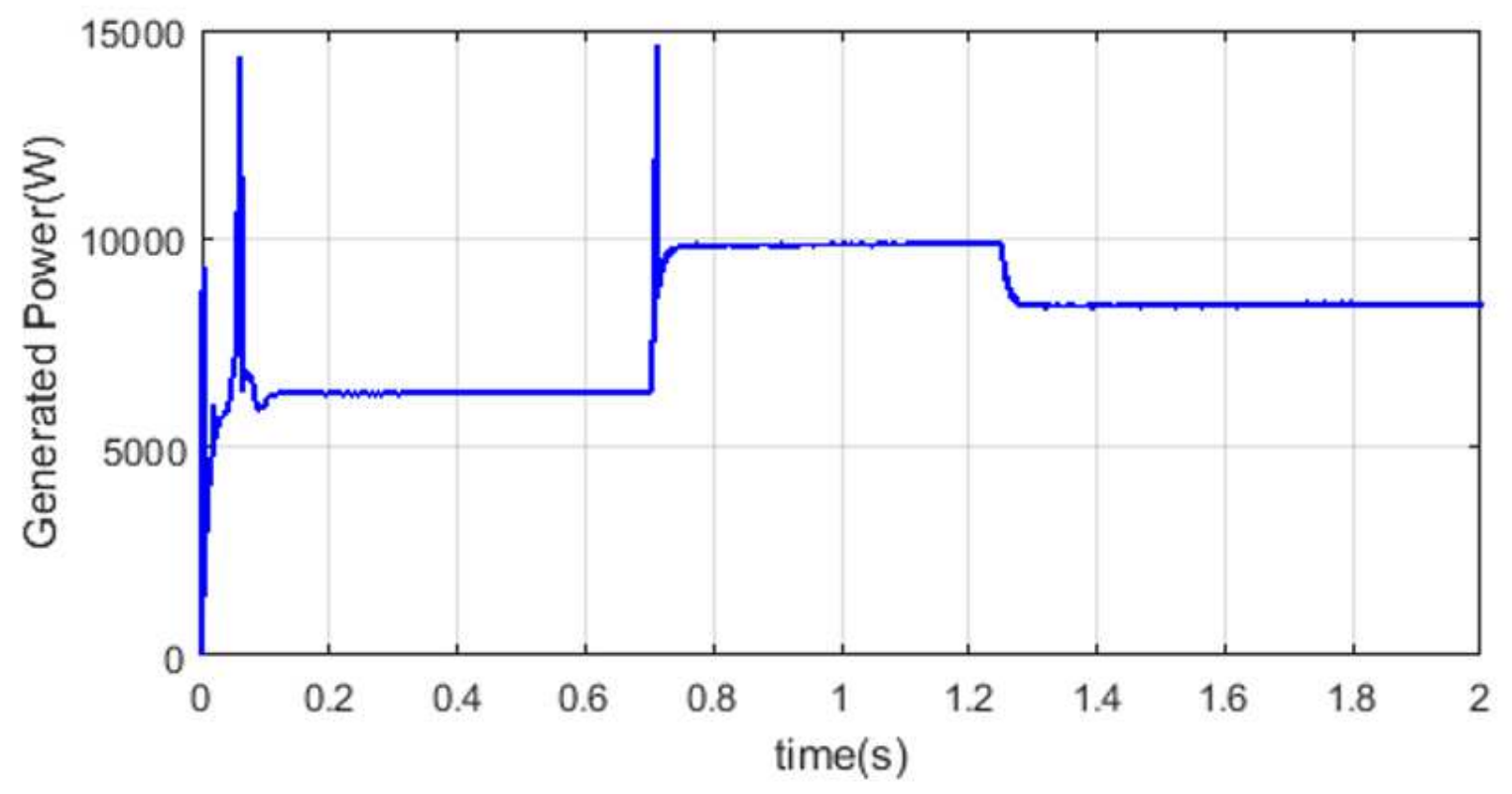

(b)

Figure 12

(a) Load Demand and (b) Generation power 


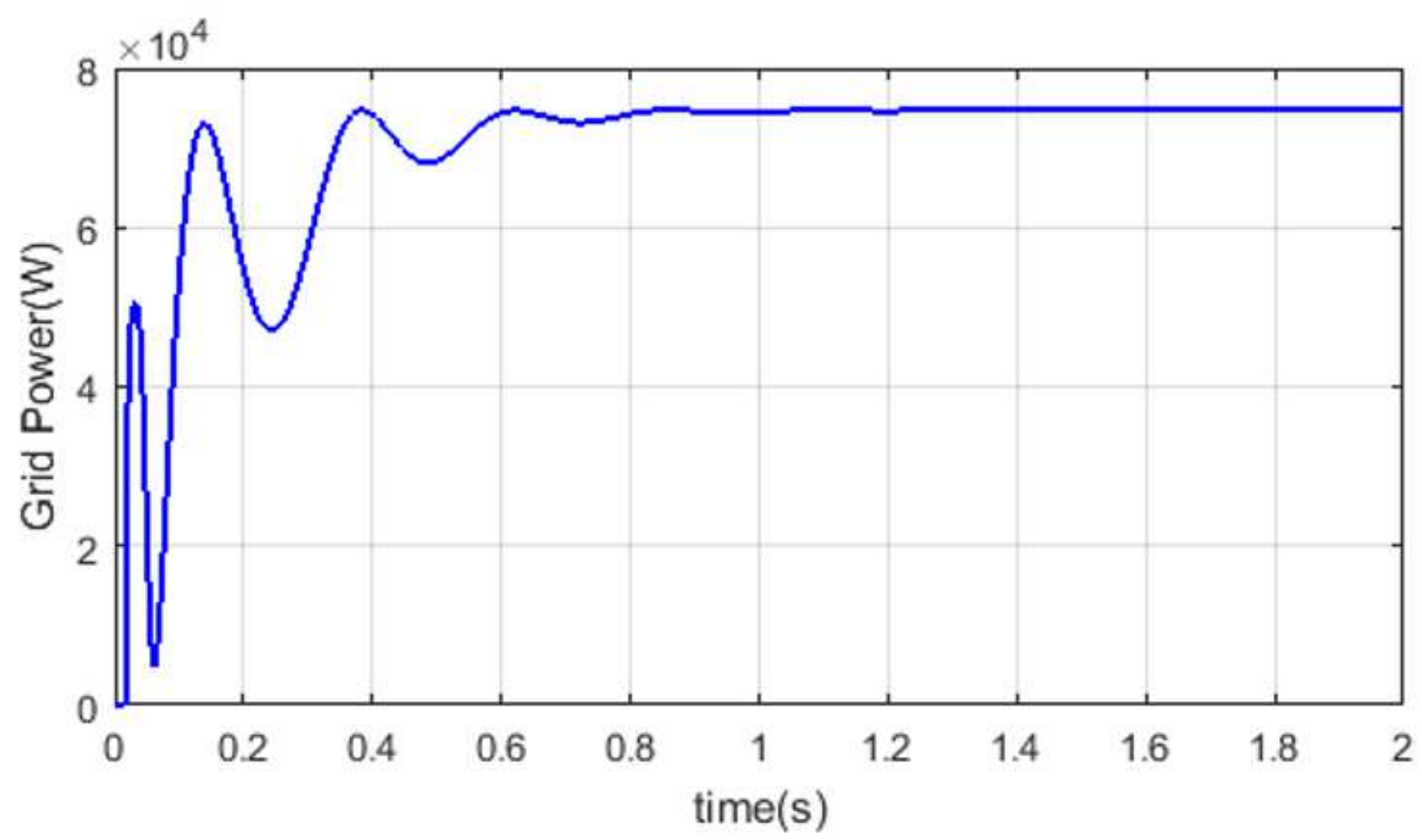

Figure 13

Analysis of the output grid power

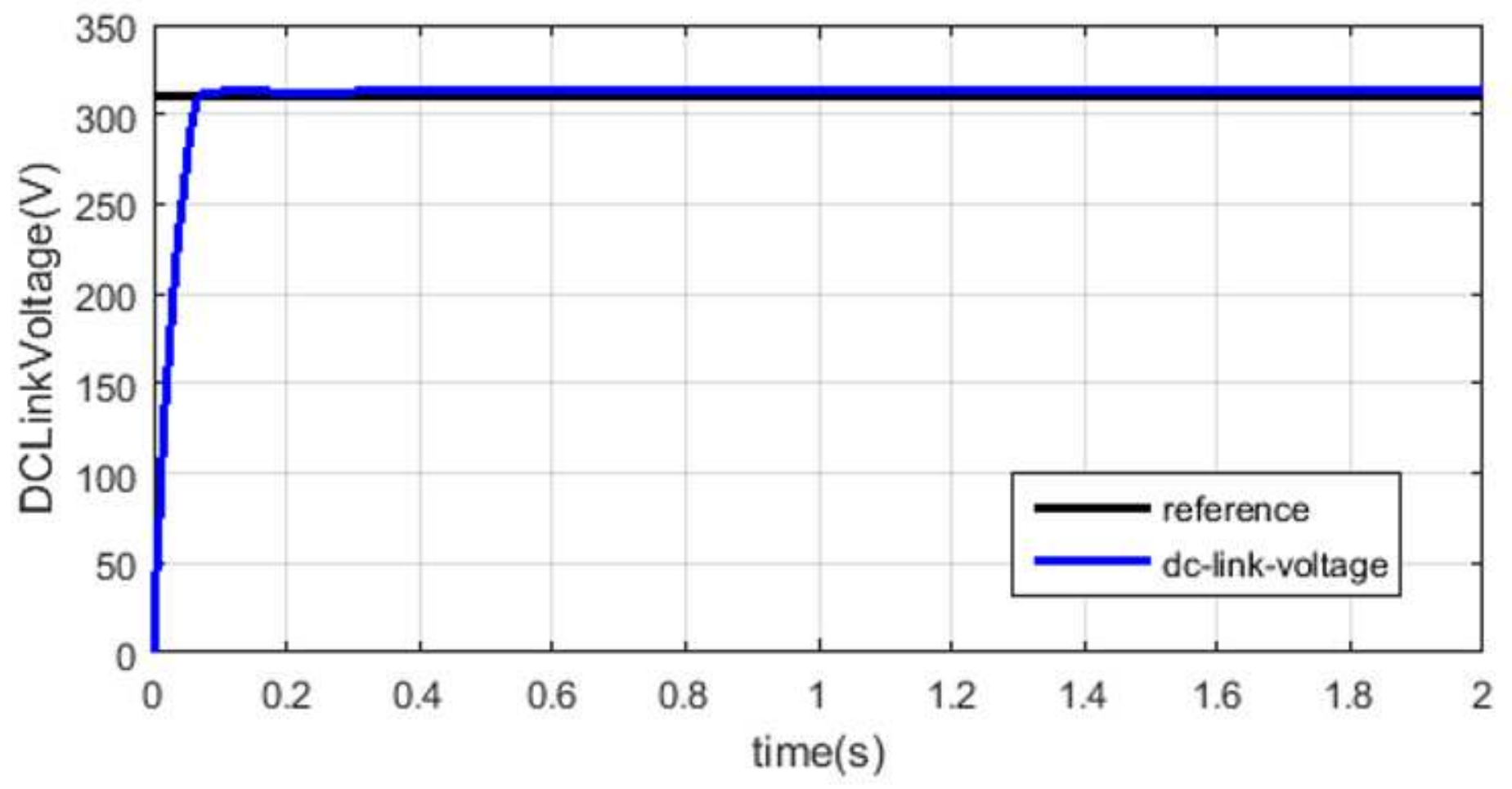

Figure 14 
Dc link voltage Analysis

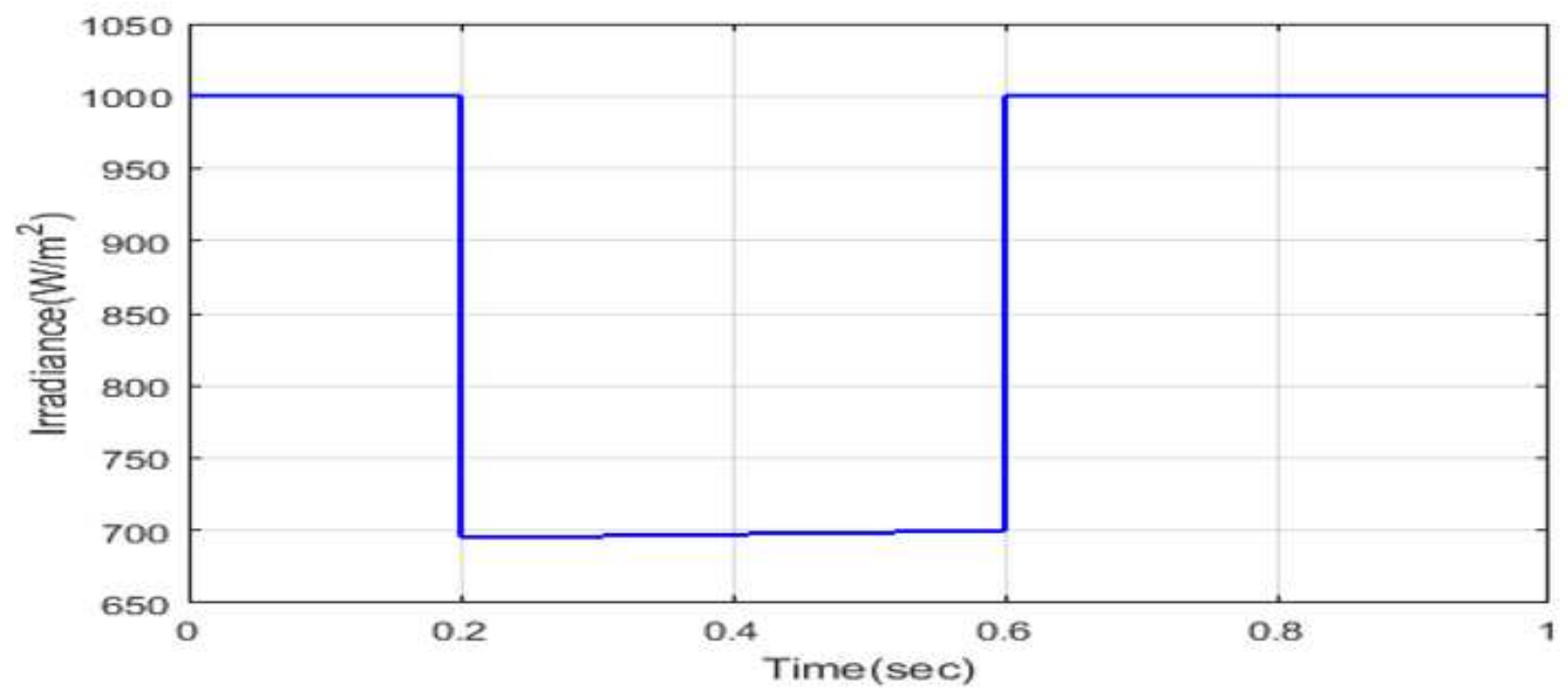

(a)

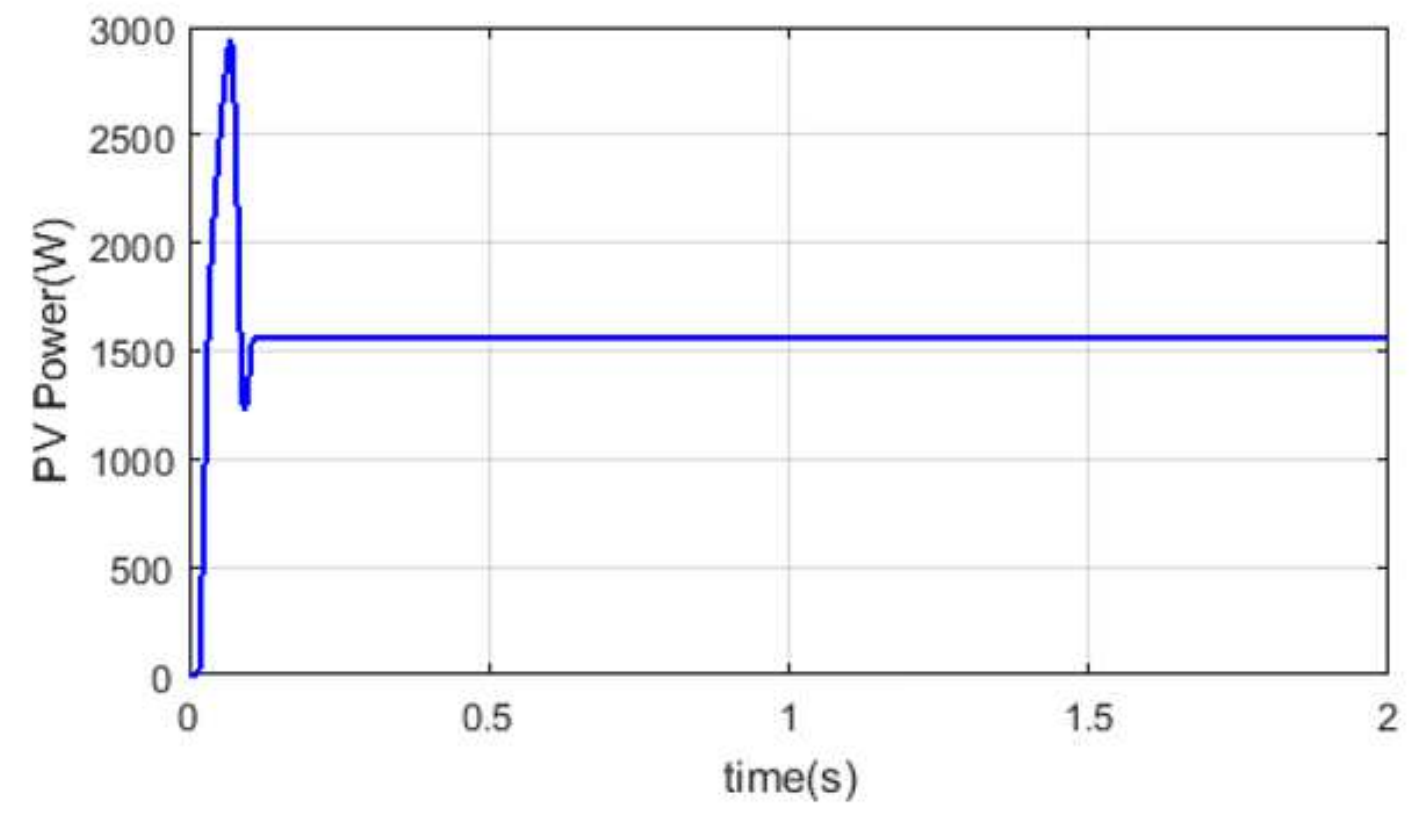

(b)

Figure 15

Solar irradiance and (b) power for PV 


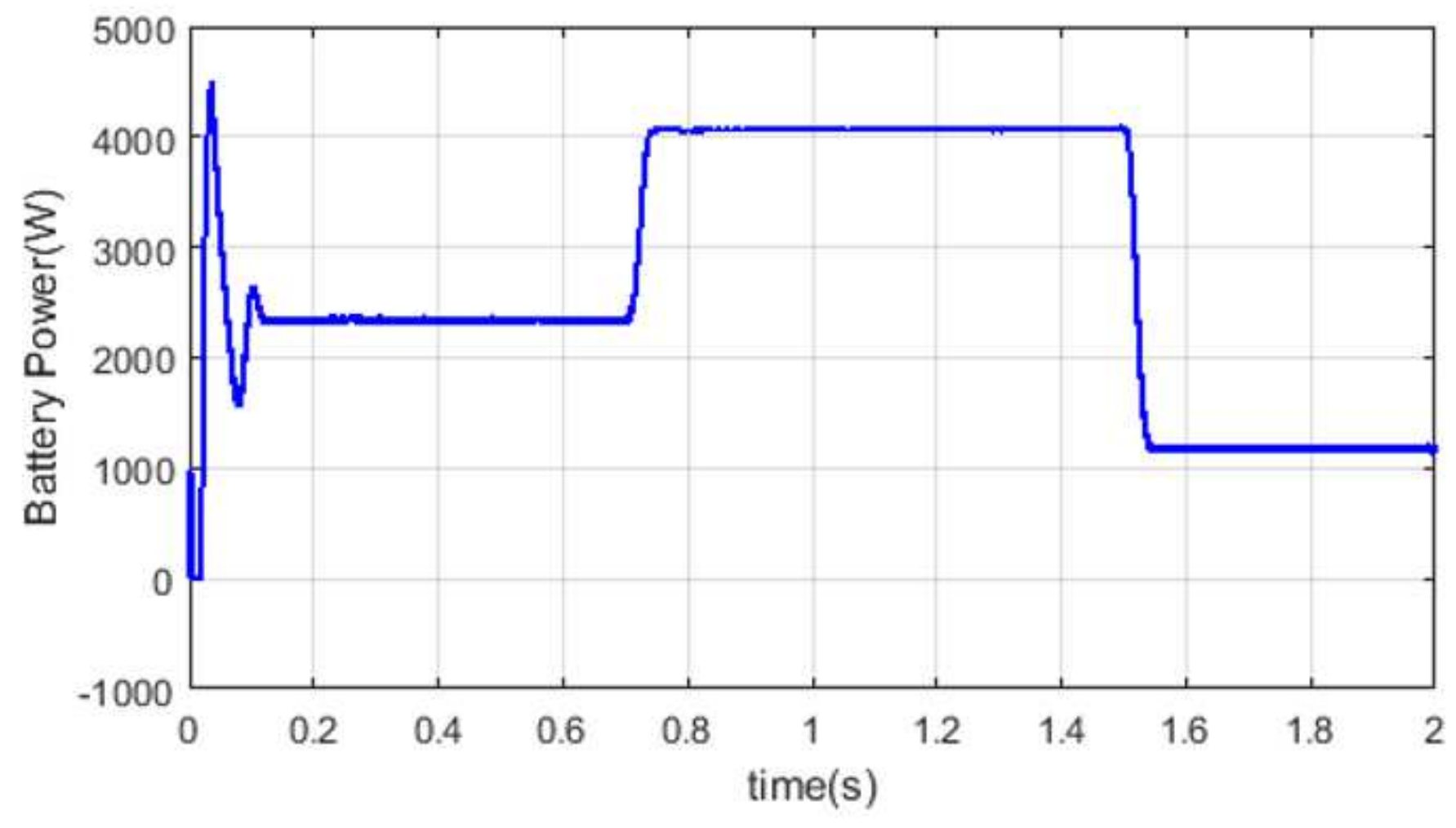

Figure 16

battery power 


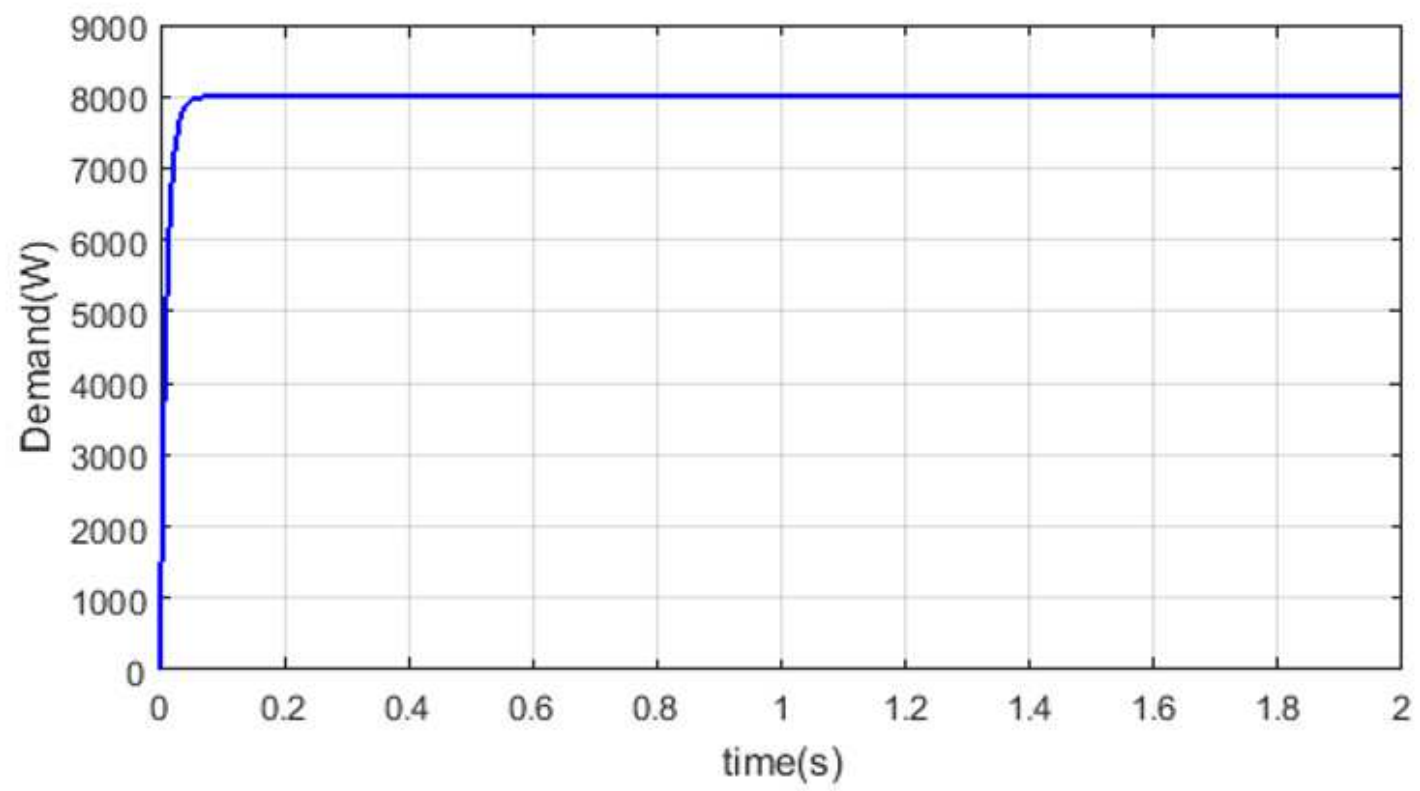

(a)

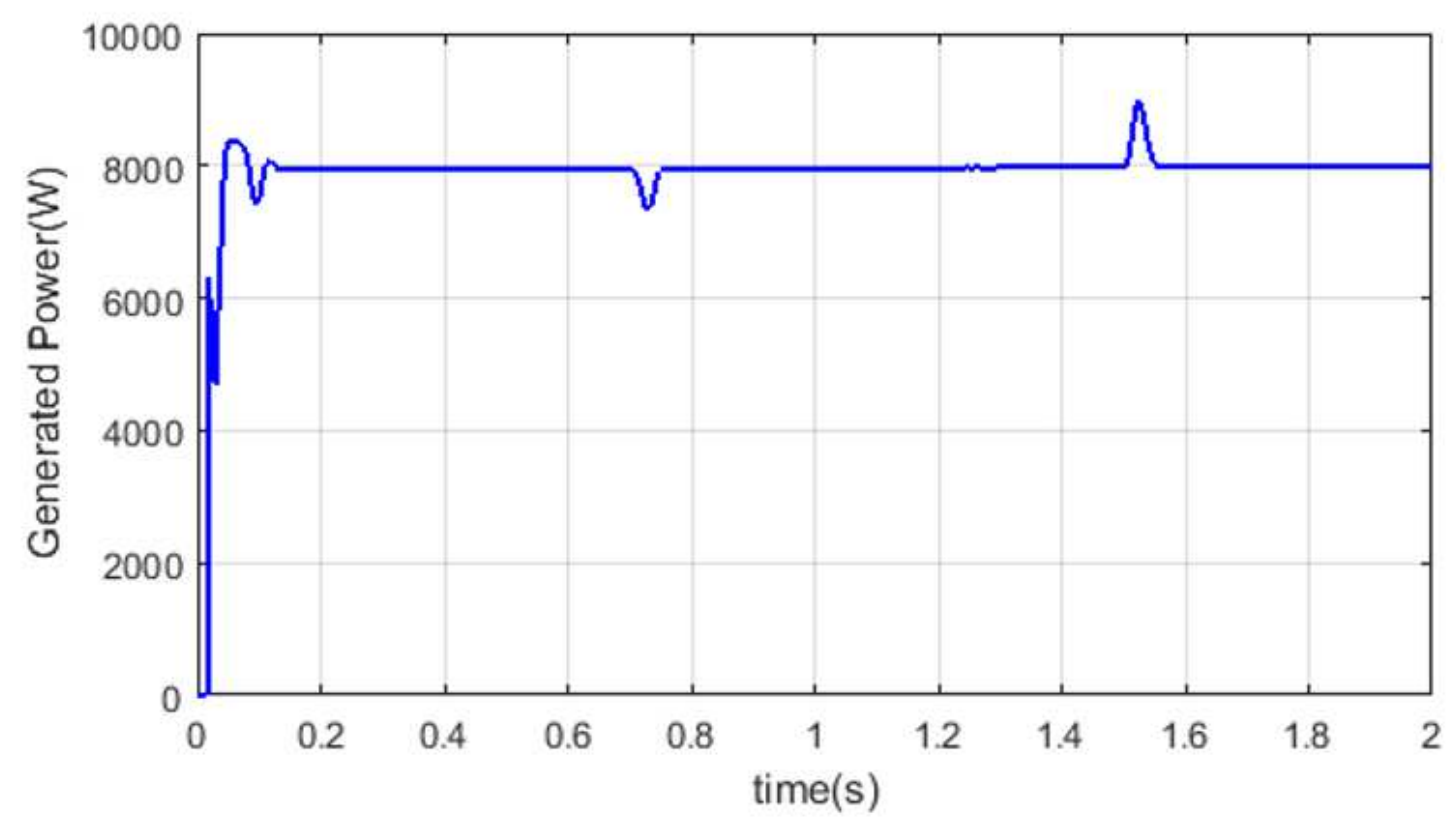

(b)

Figure 17

(a) load request and (b) generated power 


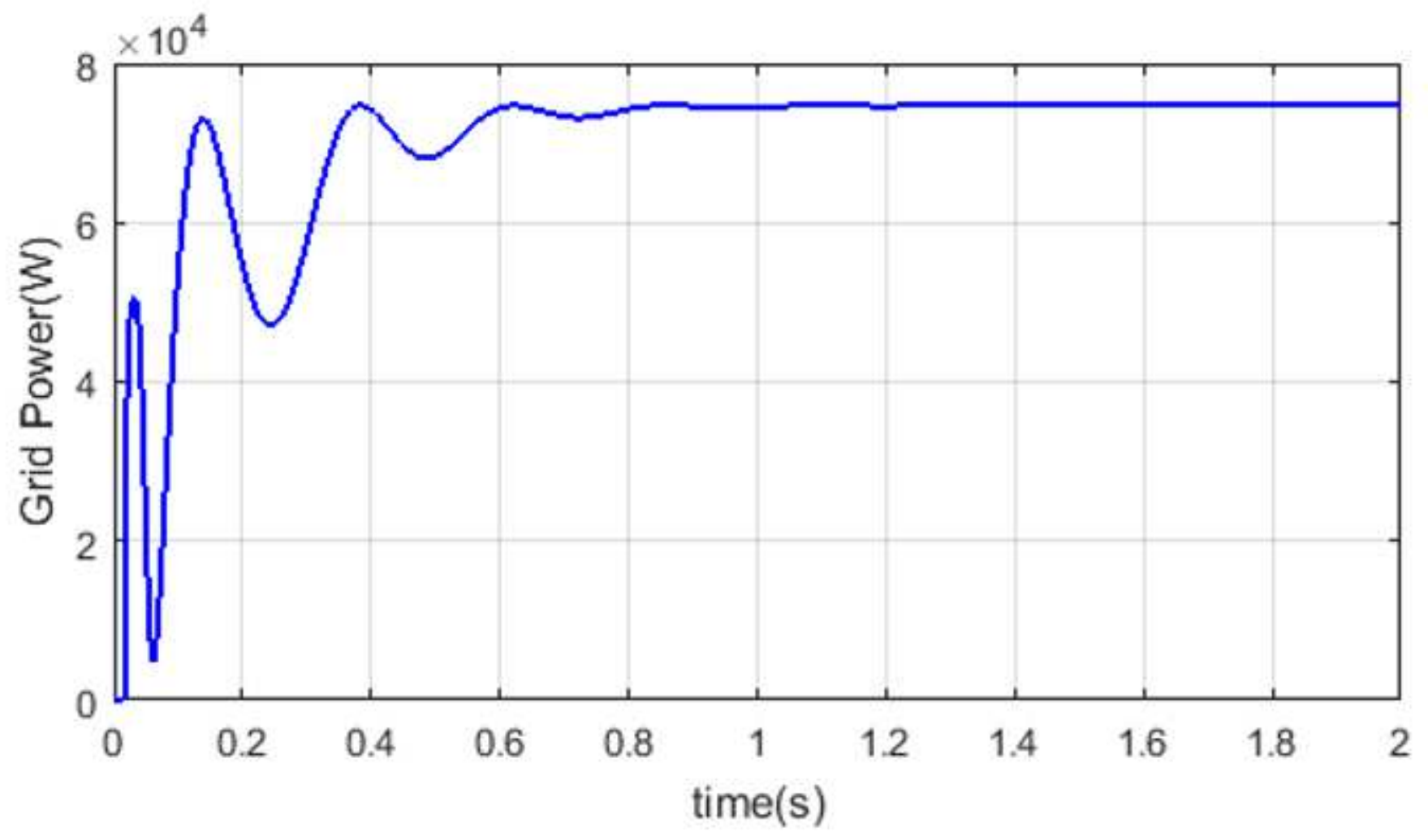

Figure 18

output power 


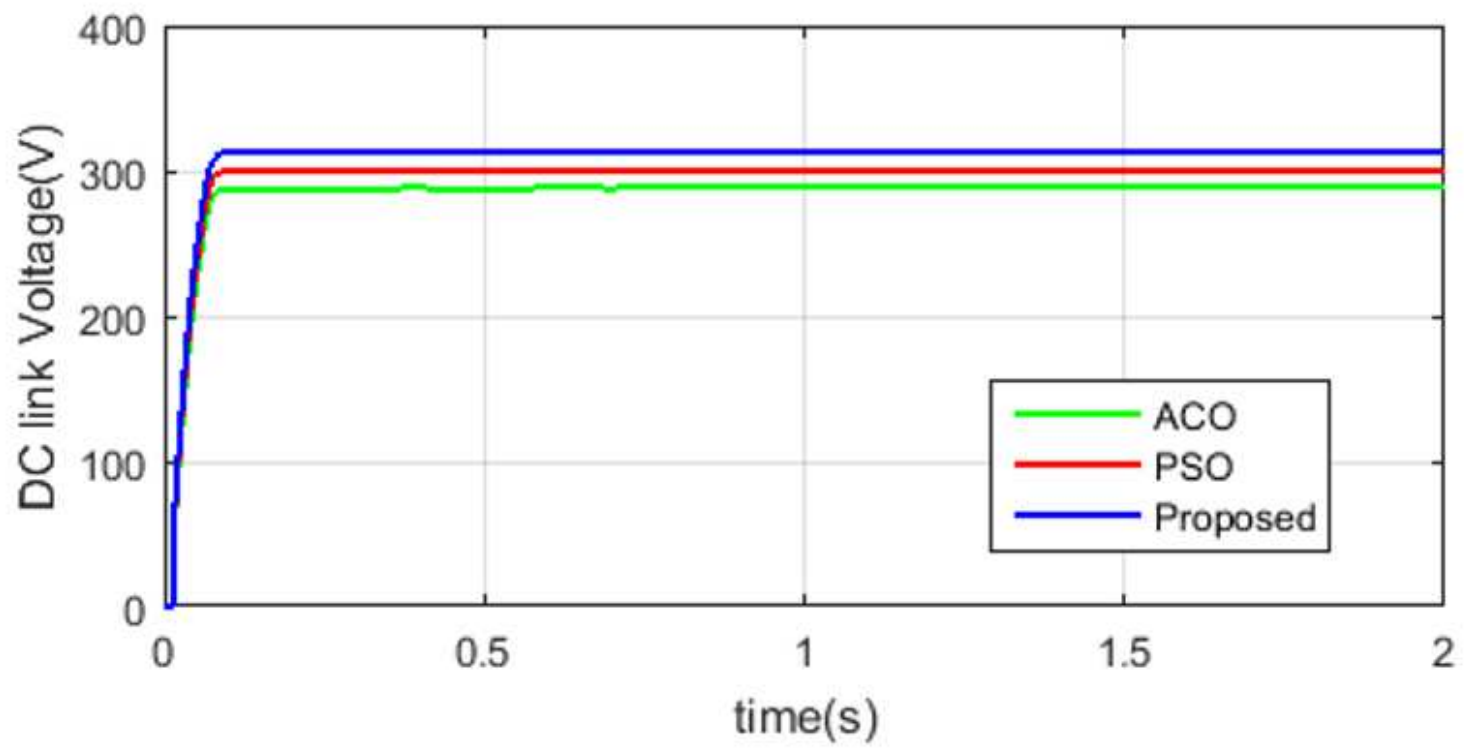

(a)

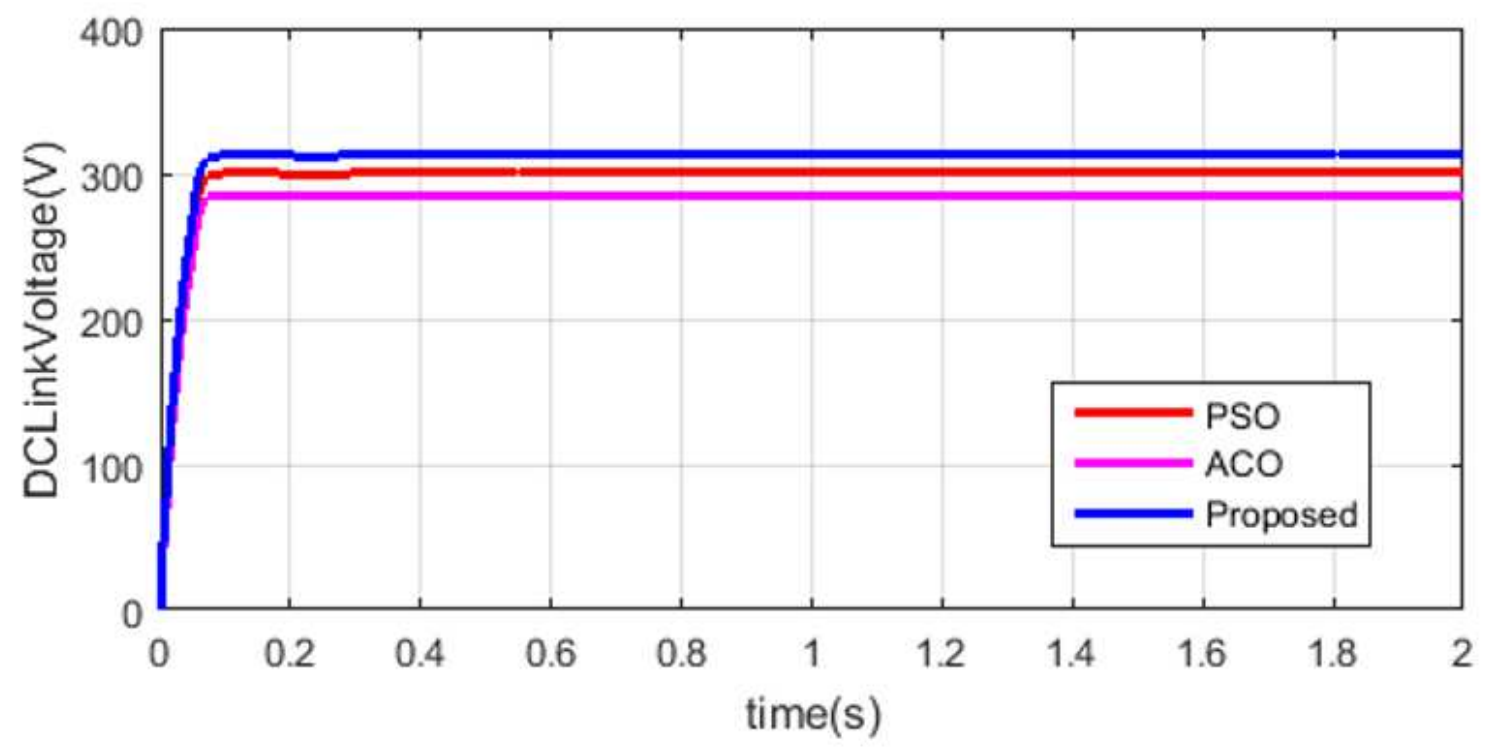

(b)

Figure 19

(a) case 1- DC link voltage and (b) case 2- DC link voltage 


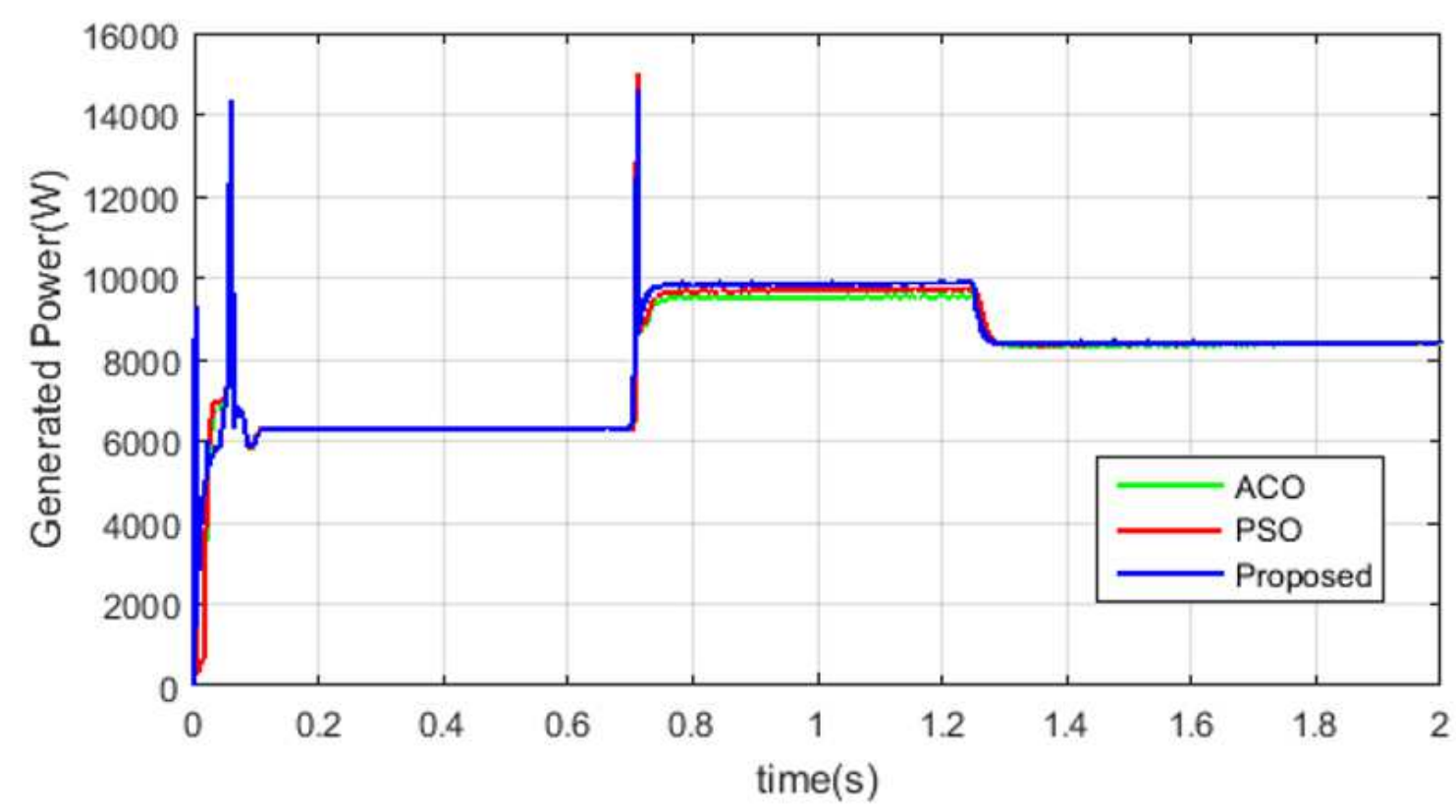

(a)

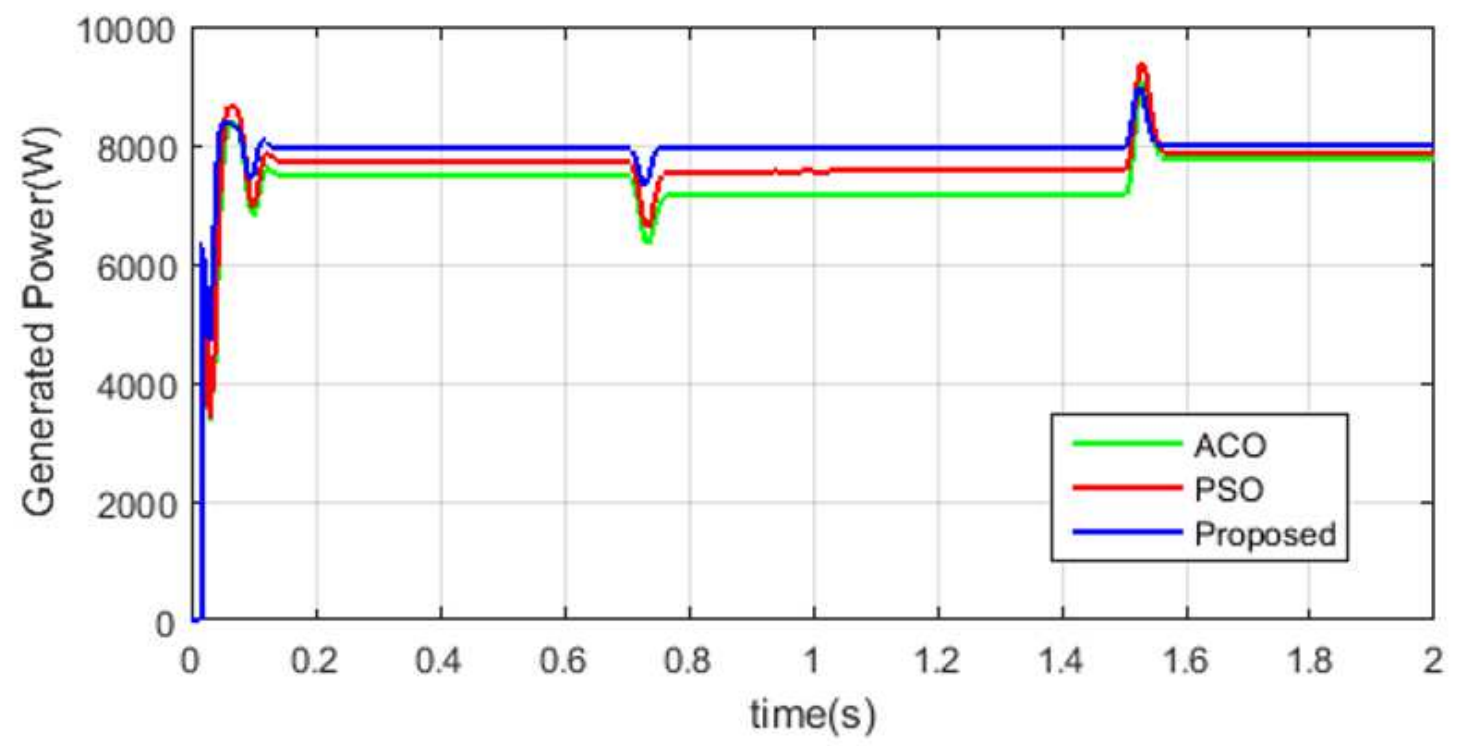

(b)

Figure 20

(a) case 1 comparison analysis and (b) case 2 comparison analysis 